FTUAM 98/8

Feb.-1999

hep-ph/9903211

\title{
THE SM AS THE QUANTUM LOW ENERGY EFFECTIVE THEORY OF THE MSSM
}

\author{
ANTONIO DOBADO \\ Departamento de Física Teórica \\ Universidad Complutense de Madrid \\ 28040-Madrid, Spain \\ MARIA J. HERRERO \\ and \\ SIANNAH PEÑARANDA \\ Departamento de Física Teórica \\ Universidad Autónoma de Madrid \\ Cantoblanco, 28049-Madrid, Spain
}

\begin{abstract}
In the framework of the Minimal Supersymmetric Standard Model we compute the one-loop effective action for the electroweak bosons obtained after integrating out the different sleptons, squarks, neutralinos and charginos, and present the result in terms of the physical sparticle masses. In addition we study the asymptotic behaviour of the two, three and four point Green's functions with external electroweak bosons in the limit where the physical sparticle masses are very large in comparison with the electroweak scale. We find that in this limit all the effects produced by the supersymmetric particles can be, either absorbed in the Standard Model parameters and gauge bosons wave functions, or else they are supressed by inverse powers of the supersymmetric particle masses. This work, therefore, completes the proof of decoupling of the heavy supersymmetric particles from the standard ones in the electroweak bosons effective action and in the sense of the Appelquist-Carazzone Theorem that we started in a previous work. From the point of view of effective field theories this work can be seen as a (partial) proof that the SM can be obtained indeed from the MSSM as the quantum low energy effective theory of this latter when the SUSY spectra is much heavier than the electroweak scale.
\end{abstract}

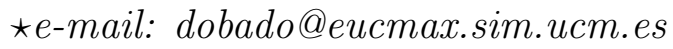

† e-mail: herrero@delta.ft.uam.es,siannah@daniel.ft.uam.es 


\section{Introduction}

In spite of the enormous amount of experimental evidence in favour of the Standard Model (SM), most of the physicists consider it just as a low energy manifestation of a more fundamental theory. Among the possible extensions of the SM one of the most popular nowdays is the so called Minimal Supersymmetric Standard Model (MSSM) [1, 2], which is the simplest theory that can be built from a supersymmetric version of the SM after the introduction of a minimal set of soft breaking terms [3]. Those terms break the supersymmetry (SUSY) of the original Supersymmetric Standard Model and give rise to contributions to the Higgs potential that finally produce the appropriate spontaneous breaking of the $S U(2)_{L} \times U(1)_{Y}$ electroweak gauge symmetry. Considering the MSSM as an interesting possibility motivated by many theoretical reasons, it is a quite natural question to ask in what sense, if any, can the SM model be considered as a low energy effective theory of the MSSM in the case where the SUSY partners of the standard particles are very heavy. In fact there are many partial indications that the SM is the low energy limit of the MSSM [4]-9]. However, most of them are based on numerical estimates and are obtained after taking some of the mass parameters appearing in the soft breaking terms numerically very large.

In this work we would like to address the question of getting the SM from the MSSM from a more formal and field theory complete point of view and, in addition, we will work directly with the physical sparticles masses instead of using the soft breaking parameters. In order to do that we will pay special attention to two esential points: First we will define in a very precise way what we understand by a low energy effective theory. The definition that we will adopt here is the one corresponding to the so called decoupling or Appelquist-Carazzone Theorem [10]. Namely, a theory with just light fields $\phi$, is considered as the low energy effective theory of a larger theory with both heavy $\tilde{\phi}$ and light $\phi$ fields if the effects of integrating out the heavy fields $\tilde{\phi}$, on the Green's functions, can be reduced to renormalizations of the parameters of the effective theory, or produce extra terms which are supressed by inverse powers of the heavy $\tilde{\phi}$ masses [11]. The second important point to be taken into account is the precise way in which the large sparticle masses limit is taken. This is essential since, due to the divergences appearing in the loop integrals, large mass limits and momentum integrations do not commute and even the large mass limit for the various particles may not commute among themselves. In this work we have choosen to take the limit where the sparticle masses $\tilde{m}_{i}$ are much larger than the electroweak boson masses and the external momenta and, at the same time, we will assume that the differences among the sparticles masses are much smaller than the sparticles masses themselves. These conditions give rise to a precise definition of the large sparticle masses regime and make it possible to define, in an unambiguous way, the resulting low energy theory for the standard particles. If this low energy theory corresponds to the SM according to the Appelquist-Carazzone definition, we will say that the SUSY particles decouple from the SM. Notice that the decoupling of the SUSY particles, in case it occurs, is neither inmediate nor trivial at all. This is because the AppelquistCarazzone Theorem does not always apply [12]-14. For example it does not apply whenever we have spontaneous symmetry breaking or chiral fermions [11]. This is just the case of the MSSM. Therefore the decoupling of the SUSY particles in the Appelquist-Carazzone sense must be showed explicitly in this case. 
Thus, our program is the following. We will start with the MSSM sector involving the electroweak bosons for which we want to study the possible decoupling of the SUSY particles. Then we compute the Green's functions for the electroweak gauge bosons that are obtained by integrating out the sleptons, squarks, neutralinos and charginos at the one-loop level (the Higgs sector of the MSSM will be considered elsewhere). Next step is the analytical study of the behaviour of these Green's functions in the asymptotic regime of the large sparticle masses defined above. This task will be much easier by using the so called m-theorem [15] as it will be explained bellow. Finally by comparison of the obtained results with the tree level SM Green's functions for the electroweak bosons, we will be able to show the decoupling of the considered SUSY particles according to the Appelquist-Carazzone definition.

The above program was started by the authors in [16], where the two point functions for electroweak gauge bosons and the $S, T$ and $U$ observables were considered. Here we continue that program and consider the three and four point functions for electroweak gauge bosons. We use the same notations and conventions for the MSSM as in our previous work that we do not repeat here for brevity. We also refer the reader to that work for more details and, in particular, for a broad discussion on the large sparticle masses limit. The present paper is organized as follows: In section two we review the definition of the low-energy action for the electroweak bosons that we presented in ref. 16. The results for the two-point functions are summarized in section three. The three and four point functions are obtained and discussed in sections four and five respectively. These and our previous results are analized together in order to establish the applicability criterion for the Appelquist-Carazzone Theorem in the studied case. Finally in section six we report the main conclusions of our work. In appendix A we define the one-loop integrals appearing in our computations by using the standard scalar and tensor integrals [17] and

give the asymptotic forms of the last ones. Appendix B contains some operators and functions which are used in this article to present the results for the three and four functions. Appendix $\mathrm{C}$ is devoted to summarize the exact results to one loop for the three and four-point sfermion contributions and for the three-point inos contributions.

\section{The low-energy effective action for the electroweak bosons}

In this section we describe our computation of the effective action for the electroweak bosons. It contains the two, three and four point Green's functions and is obtained after the integration of the sfermions and inos, viz. charginos and neutralinos of the MSSM [16.

In more generic words, our aim is to compute the effective action $\Gamma_{\text {eff }}[\phi]$ for the standard particles $\phi$ that is defined through functional integration of all the sparticles of the MSSM $\tilde{\phi}$. In short notation it is defined by,

$$
\mathrm{e}^{i \Gamma_{e f f}[\phi]}=\int[\mathrm{d} \tilde{\phi}] \mathrm{e}^{i \Gamma_{\mathrm{MSSM}}[\phi, \tilde{\phi}]}
$$


with

$$
\Gamma_{\operatorname{MSSM}}[\phi, \tilde{\phi}] \equiv \int d x \mathcal{L}_{\mathrm{MSSM}}(\phi, \tilde{\phi}) ; \mathrm{d} x \equiv \mathrm{d}^{4} x
$$

and $\mathcal{L}_{\text {MSSM }}$ is the MSSM Lagrangian. The computation of the effective action will be performed at the one loop level by using dimensional regularization, in an arbitrary $R_{\xi}$ gauge and will include the integration of all the sfermions $\tilde{f}$ (squarks $\tilde{q}$ and sleptons $\tilde{l}$ ), neutralinos $\tilde{\chi}^{o}$ and charginos $\tilde{\chi}^{+}$. Our program starts, in particular, with the computation of the electroweak gauge boson effective action $\Gamma_{\operatorname{MSSM}}[V]\left(V=A, Z\right.$ and $\left.W^{ \pm}\right)$given by,

$$
\mathrm{e}^{i \Gamma_{e f f}[V]}=\int[\mathrm{d} \tilde{f}]\left[\mathrm{d} \tilde{f}^{*}\right]\left[\mathrm{d} \tilde{\chi}^{+}\right]\left[\mathrm{d} \overline{\tilde{\chi}}^{+}\right]\left[\mathrm{d} \tilde{\chi}^{o}\right] \mathrm{e}^{i \Gamma_{\mathrm{MSSM}}\left[V, \tilde{f}, \tilde{\chi}^{+}, \tilde{\chi}^{o}\right]}
$$

where,

$$
\begin{aligned}
\Gamma_{\mathrm{MSSM}}\left[V, \tilde{f}, \tilde{\chi}^{+}, \tilde{\chi}^{o}\right] & \equiv \int d x \mathcal{L}_{\mathrm{MSSM}}\left(V, \tilde{f}, \tilde{\chi}^{+}, \tilde{\chi}^{o}\right) \\
& =\int d x \mathcal{L}^{(0)}(V)+\int d x \mathcal{L}_{\tilde{f}}(V, \tilde{f})+\int d x \mathcal{L}_{\tilde{\chi}}(V, \tilde{\chi}) \\
& \equiv \Gamma_{0}[V]+\Gamma_{\tilde{f}}[V, \tilde{f}]+\Gamma_{\tilde{\chi}}[V, \tilde{\chi}]
\end{aligned}
$$

and $\mathcal{L}^{(0)}, \mathcal{L}_{\tilde{f}}, \mathcal{L}_{\tilde{\chi}}$ are the free Lagrangian and the interaction Lagrangian of gauge bosons with sfermions and inos respectively. From now on we will follow closely the definitions, notations and conventions introduced in [16]. In particular we will use the compact notation :

$$
\begin{aligned}
& \phi(x) \equiv \phi_{x}, \delta(x-y) \equiv \delta_{x y}, A(x, y) \equiv A_{x y} \\
& \operatorname{Tr} A=\operatorname{tr} \int d x A_{x x}=\sum_{a} \int d x A_{x x}^{a a}
\end{aligned}
$$

and

$$
\tilde{f} \equiv\left(\begin{array}{c}
\tilde{t}_{1} \\
\tilde{t}_{2} \\
\tilde{b}_{1} \\
\tilde{b}_{2}
\end{array}\right) \quad \text { if } \tilde{f}=\tilde{q} ; \quad \tilde{f} \equiv\left(\begin{array}{c}
\tilde{\nu} \\
0 \\
\tilde{\tau}_{1} \\
\tilde{\tau}_{2}
\end{array}\right) \quad \text { if } \quad \tilde{f}=\tilde{l}, \quad \tilde{\chi}^{+} \equiv\left(\begin{array}{c}
\tilde{\chi}_{1}^{+} \\
\tilde{\chi}_{2}^{+}
\end{array}\right), \quad \tilde{\chi}^{o} \equiv\left(\begin{array}{c}
\tilde{\chi}_{1}^{o} \\
\tilde{\chi}_{2}^{o} \\
\tilde{\chi}_{3}^{o} \\
\tilde{\chi}_{4}^{o}
\end{array}\right)
$$

The actions appearing in eq. (四) can be written as

$$
\Gamma_{\tilde{f}}[V, \tilde{f}]=\left\langle\tilde{f}^{+} A_{\tilde{f}} \tilde{f}\right\rangle
$$

where,

$$
\begin{aligned}
A_{\tilde{f}} & \equiv A_{\tilde{f}}^{(0)}+A_{\tilde{f}}^{(1)}+A_{\tilde{f}}^{(2)} \\
\left\langle\tilde{f}^{+} A_{\tilde{f}}^{(i)} \tilde{f}\right\rangle & \equiv \sum_{\tilde{f}} \int d x d y \tilde{f}_{x}^{+} A_{\tilde{f} x y}^{(i)} \tilde{f}_{y}, i=0,1,2
\end{aligned}
$$


and the operators are:

$$
\begin{aligned}
A_{\tilde{f} x y}^{(0)} & \equiv\left(-\square-\tilde{M}_{f}^{2}\right)_{x} \delta_{x y} \\
A_{\tilde{f} x y}^{(1)} & \equiv-i e\left(\partial_{\mu} A^{\mu} \hat{Q}_{f}+2 \hat{Q}_{f} A_{\mu} \partial^{\mu}\right)_{x} \delta_{x y}-\frac{i g}{c_{w}}\left(\partial_{\mu} Z^{\mu} \hat{G}_{f}+2 \hat{G}_{f} Z_{\mu} \partial^{\mu}\right)_{x} \delta_{x y} \\
& -\frac{i g}{\sqrt{2}}\left(\partial_{\mu} W^{+\mu} \Sigma_{f}^{t b}+2 \Sigma_{f}^{t b} W_{\mu}^{+} \partial^{\mu}\right)_{x} \delta_{x y}+\text { h.c. } \\
A_{\tilde{f} x y}^{(2)} & \equiv\left(e^{2} \hat{Q}_{f}^{2} A_{\mu} A^{\mu}+\frac{2 g e}{c_{W}} A_{\mu} Z^{\mu} \hat{Q}_{f} \hat{G}_{f}+\frac{g^{2}}{c_{W}^{2}} \hat{G}_{f}^{2} Z_{\mu} Z^{\mu}+\frac{1}{2} g^{2} \Sigma_{f} W_{\mu}^{+} W^{\mu-}\right. \\
& +\frac{e g}{\sqrt{2}} y_{f} A_{\mu} W^{\mu+} \Sigma_{f}^{t b}+\frac{e g}{\sqrt{2}} y_{f} A_{\mu} W^{\mu-} \Sigma_{f}^{b t} \\
& \left.-\frac{g^{2}}{\sqrt{2}} y_{f} \frac{s_{W}^{2}}{c_{W}} Z_{\mu} W^{\mu+} \Sigma_{f}^{t b}-\frac{g^{2}}{\sqrt{2}} y_{f} \frac{s_{W}^{2}}{c_{W}} Z_{\mu} W^{\mu-} \Sigma_{f}^{b t}\right)_{x} \delta_{x y}
\end{aligned}
$$

where $s_{W}^{2}=\sin ^{2} \theta_{W}, c_{W}^{2}=\cos ^{2} \theta_{W}$ and $y_{f}=\frac{1}{3}$ if $\tilde{f}=\tilde{q}$ or $y_{f}=-1$ if $\tilde{f}=\tilde{l}$.

Analogously we have

$$
\Gamma_{\tilde{\chi}}[V, \tilde{\chi}]=\frac{1}{2}\left\langle\overline{\tilde{\chi}}^{o}\left(A_{0}^{(0)}+A_{0}^{(1)}\right) \tilde{\chi}^{o}\right\rangle+\left\langle\overline{\tilde{\chi}}^{+}\left(A_{+}^{(0)}+A_{+}^{(1)}\right) \tilde{\chi}^{+}\right\rangle+\left\langle\overline{\tilde{\chi}}^{o} A_{0+}^{(1)} \tilde{\chi}^{+}\right\rangle+\left\langle\overline{\tilde{\chi}}^{+} A_{+0}^{(1)} \tilde{\chi}^{o}\right\rangle
$$

where

$$
\begin{aligned}
\left\langle\overline{\tilde{\chi}}^{o} A_{0}^{(i)} \tilde{\chi}^{o}\right\rangle & \equiv \int d x d y \overline{\tilde{\chi}}_{x}^{o} A_{0 x y}^{(i)} \tilde{\chi}_{y}^{o}, \quad\left\langle\overline{\tilde{\chi}}^{+} A_{+}^{(i)} \tilde{\chi}^{+}\right\rangle \equiv \int d x d y \overline{\tilde{\chi}}_{x}^{+} A_{+x y}^{(i)} \tilde{\chi}_{y}^{+} ; i=0,1 \\
\left\langle\overline{\tilde{\chi}}^{o} A_{0+}^{(1)} \tilde{\chi}^{+}\right\rangle & \equiv \int d x d y \overline{\tilde{\chi}}_{x}^{o} A_{0+x y}^{(1)} \tilde{\chi}_{y}^{+},\left\langle\overline{\tilde{\chi}}^{+} A_{+0}^{(1)} \tilde{\chi}^{o}\right\rangle \equiv \int d x d y \overline{\tilde{\chi}}_{x}^{+} A_{+0 x y}^{(1)} \tilde{\chi}_{y}^{o}
\end{aligned}
$$

and the operators are:

$$
\begin{aligned}
A_{0 x y}^{(0)} & \equiv\left(i \not \partial-\tilde{M}^{0}\right)_{x} \delta_{x y}, \quad A_{+x y}^{(0)} \equiv\left(i \not \partial-\tilde{M}^{+}\right)_{x} \delta_{x y} \\
A_{0 x y}^{(1)} & \equiv \frac{g}{c_{w}} Z_{\mu} \gamma^{\mu}\left(O_{L}^{\prime \prime} P_{L}+O_{R}^{\prime \prime} P_{R}\right)_{x} \delta_{x y}, A_{+x y}^{(1)} \equiv\left[\frac{g}{c_{w}} Z_{\mu} \gamma^{\mu}\left(O_{L}^{\prime} P_{L}+O_{R}^{\prime} P_{R}\right)-e A_{\mu} \gamma^{\mu}\right]_{x} \delta_{x y} \\
A_{0+x y}^{(1)} & \equiv\left[g W_{\mu}^{-} \gamma^{\mu}\left(O_{L} P_{L}+O_{R} P_{R}\right)\right]_{x} \delta_{x y}, \quad A_{+0 x y}^{(1)} \equiv\left[g W_{\mu}^{+} \gamma^{\mu}\left(O_{L}^{+} P_{L}+O_{R}^{+} P_{R}\right)\right]_{x} \delta_{x y} .
\end{aligned}
$$

In the above expressions the coupling matrices $\hat{Q}_{f}, \hat{G}_{f}, \Sigma_{f}^{t b}, \Sigma_{f}^{b t}, \Sigma_{f}$, and $O_{L}, O_{R}, O_{L}^{\prime}, O_{R}^{\prime}, O_{L}^{\prime \prime}, O_{R}^{\prime \prime}$ as well as the mass matrices $\tilde{M}_{f}, \tilde{M}^{0}$ and $\tilde{M}^{+}$are defined in [16].

The effective action can be written as:

$$
e^{i \Gamma_{e f f}[V]}=e^{i \Gamma_{o}[V]} e^{i \Gamma_{e f f}^{\tilde{f}}[V]} e^{i \Gamma_{e f f}^{\tilde{\chi}}[V]}
$$

where,

$$
\begin{gathered}
e^{i \Gamma_{e f f}^{\tilde{f}}[V]}=\int[d \tilde{f}]\left[d \tilde{f}^{*}\right] e^{i \Gamma_{\tilde{f}}[V, \tilde{f}]} \\
e^{i \Gamma_{e f f}^{\tilde{\chi}}[V]}=\int\left[d \tilde{\chi}^{+}\right]\left[d \overline{\tilde{\chi}}^{+}\right]\left[d \tilde{\chi}^{o}\right] e^{i \Gamma_{\tilde{\chi}}[V, \tilde{\chi}]}
\end{gathered}
$$


After a Gaussian integration on the complex sfermion fields we find,

$$
\Gamma_{e f f}^{\tilde{f}}[V]=i \operatorname{Tr} \log A_{\tilde{f}}=i \operatorname{Tr} \log \left[A_{\tilde{f}}^{(o)}\left(1+A_{\tilde{f}}^{(o)-1}\left(A_{\tilde{f}}^{(1)}+A_{\tilde{f}}^{(2)}\right)\right)\right]
$$

and by making the standard manipulations we get

$$
\Gamma_{e f f}^{\tilde{f}}[V]=i \sum_{k=1}^{\infty} \frac{(-1)^{k+1}}{k} \operatorname{Tr}\left[G_{\tilde{f}}\left(A_{\tilde{f}}^{(1)}+A_{\tilde{f}}^{(2)}\right)\right]^{k}
$$

where the free sfermion propagator matrix $G_{\tilde{f}} \equiv A_{\tilde{f}}^{(o)^{-1}}$ is given by

$$
G_{\tilde{f} x y}^{i j} \equiv \int \frac{d^{D} q}{(2 \pi)^{D}} \mu_{o}^{4-D} e^{-i q(x-y)}\left(q^{2}-\tilde{M}_{f}^{2}\right)_{i j}^{-1}
$$

with,

$$
\begin{aligned}
& \left(q^{2}-\tilde{M}_{f}^{2}\right)^{-1}=\operatorname{diag}\left(\frac{1}{q^{2}-\tilde{m}_{t_{1}}^{2}}, \frac{1}{q^{2}-\tilde{m}_{t_{2}}^{2}}, \frac{1}{q^{2}-\tilde{m}_{b_{1}}^{2}}, \frac{1}{q^{2}-\tilde{m}_{b_{2}}^{2}}\right) \quad \text { if } \quad \tilde{f}=\tilde{q} \quad ; \text { or } \\
& \left(q^{2}-\tilde{M}_{f}^{2}\right)^{-1}=\operatorname{diag}\left(\frac{1}{q^{2}-\tilde{m}_{\nu}^{2}}, \frac{1}{q^{2}}, \frac{1}{q^{2}-\tilde{m}_{\tau_{1}}^{2}}, \frac{1}{q^{2}-\tilde{m}_{\tau_{2}}^{2}}\right) \quad \text { if } \quad \tilde{f}=\tilde{l}
\end{aligned}
$$

and the sums over the three generations and the $N_{c}$ squarks colors are implicit. Finally, if we keep just the terms that contribute to the two, three and four point functions, the effective action generated from sfermions integration can be written as,

$$
\begin{aligned}
\Gamma_{e f f}^{\tilde{f}}[V] & =i \operatorname{Tr}\left(G_{\tilde{f}} A_{\tilde{f}}^{(2)}\right)-\frac{i}{2} \operatorname{Tr}\left(G_{\tilde{f}} A_{\tilde{f}}^{(1)}\right)^{2}-i \operatorname{Tr}\left(G_{\tilde{f}} A_{\tilde{f}}^{(1)} G_{\tilde{f}} A_{\tilde{f}}^{(2)}\right)+\frac{i}{3} \operatorname{Tr}\left(G_{\tilde{f}} A_{\tilde{f}}^{(1)}\right)^{3} \\
& -\frac{i}{2} \operatorname{Tr}\left(G_{\tilde{f}} A_{\tilde{f}}^{(2)}\right)^{2}+i \operatorname{Tr}\left(G_{\tilde{f}} A_{\tilde{f}}^{(1)} G_{\tilde{f}} A_{\tilde{f}}^{(1)} G_{\tilde{f}} A_{\tilde{f}}^{(2)}\right)-\frac{i}{4} \operatorname{Tr}\left(G_{\tilde{f}} A_{\tilde{f}}^{(1)}\right)^{4}+O\left(V^{5}\right),
\end{aligned}
$$

Clearly, we can identify the first and second terms in eq. (18) with the one-loop contributions from sfermions to the two-point functions; the third and fourth terms with the contributions to the three-point functions and the last three terms are the corresponding contributions to the four-point functions.

On the other hand the contributions to the electroweak gauge bosons effective action comming from the neutralinos and the charginos are given by:

$$
e^{i \Gamma_{e f f}^{\tilde{\chi}}[V]}=\int\left[d \tilde{\chi}^{+}\right]\left[d \overline{\tilde{\chi}}^{+}\right]\left[d \tilde{\chi}^{o}\right] e^{i\left\{\frac{1}{2}<\bar{\chi}^{o}\left(A_{o}^{(o)}+A_{o}^{(1)}\right) \tilde{\chi}^{o}>+<\bar{\chi}^{+}\left(A_{+}^{(o)}+A_{+}^{(1)}\right) \tilde{\chi}^{+}>+<\bar{\chi}^{o} A_{o+}^{(1)} \tilde{\chi}^{+}>+<\bar{\chi}^{+} A_{o+}^{(1)} \tilde{\chi}^{o}>\right\}}
$$

By performing first a standard Grassmann integration on the chargino fields we find,

$$
e^{i \Gamma_{e f f}^{\tilde{\chi}}[V]}=\operatorname{det}\left(A_{+}^{(o)}+A_{+}^{(1)}\right) \int\left[d \tilde{\chi}^{o}\right] e^{i \frac{1}{2}<\overline{\tilde{\chi}}^{o}\left[A_{o}^{(o)}+A_{o}^{(1)}-2 A_{o+}^{(1)}\left(A_{+}^{(o)}+A_{+}^{(1)}\right)^{-1} A_{+o}^{(1)}\right] \tilde{\chi}^{o}>}
$$

Next we integrate over the neutralinos which are Majorana fermion fields and find,

$$
e^{i \Gamma_{e f f}^{\tilde{\tilde{f}}[V]}}=\operatorname{det}\left(A_{+}^{(o)}+A_{+}^{(1)}\right) \operatorname{det}\left[A_{o}^{(o)}+A_{o}^{(1)}-2 A_{o+}^{(1)}\left(A_{+}^{(o)}+A_{+}^{(1)}\right)^{-1} A_{+o}^{(1)}\right]^{\frac{1}{2}} .
$$


So that the effective action can be written as,

$$
\Gamma_{e f f}^{\tilde{\tilde{\chi}}}[V]=-i \operatorname{Tr} \log \left(A_{+}^{(o)}+A_{+}^{(1)}\right)-\frac{i}{2} \operatorname{Tr} \log \left[A_{o}^{(o)}+A_{o}^{(1)}-2 A_{o+}^{(1)}\left(A_{+}^{(o)}+A_{+}^{(1)}\right)^{-1} A_{+o}^{(1)}\right]
$$

Now, by introducing the chargino propagator $k_{+} \equiv A_{+}^{(o)^{-1}}$ which is given by the matrix

$$
k_{+x y}^{i j} \equiv \int \frac{d^{D} q}{(2 \pi)^{D}} \mu_{o}^{4-D} e^{-i q(x-y)}\left(\not 1-\tilde{M}_{+}\right)_{i j}^{-1}, \quad i, j=1,2 .
$$

and the neutralino propagator $k_{o} \equiv A_{o}^{(o)^{-1}}$ which is given by the matrix

$$
k_{o x y}^{i j} \equiv \int \frac{d^{D} q}{(2 \pi)^{D}} \mu_{o}^{4-D} e^{-i q(x-y)}\left(\not q-\tilde{M}_{o}\right)_{i j}^{-1}, \quad i, j=1,2,3,4
$$

we can writte the total inos contribution to the effective action as

$$
\begin{aligned}
\Gamma_{e f f}^{\tilde{\chi}}[V] & =\frac{i}{2} \operatorname{Tr}\left(k_{+} A_{+}^{(1)}\right)^{2}+\frac{i}{4} \operatorname{Tr}\left(k_{o} A_{o}^{(1)}\right)^{2}+i \operatorname{Tr}\left(k_{o} A_{o+}^{(1)} k_{+} A_{+o}^{(1)}\right)-\frac{i}{3} \operatorname{Tr}\left(k_{+} A_{+}^{(1)}\right)^{3} \\
& -i \operatorname{Tr}\left(k_{o} A_{o+}^{(1)} k_{+} A_{+}^{(1)} k_{+} A_{+o}^{(1)}\right)-i \operatorname{Tr}\left(k_{o} A_{o}^{(1)} k_{o} A_{o+}^{(1)} k_{+} A_{+o}^{(1)}\right)-\frac{i}{6} \operatorname{Tr}\left(k_{o} A_{o}^{(1)}\right)^{3} \\
& +\frac{i}{4} \operatorname{Tr}\left(k_{+} A_{+}^{(1)}\right)^{4}+i \operatorname{Tr}\left(k_{o} A_{o+}^{(1)}\left(k_{+} A_{+}^{(1)}\right)^{2} k_{+} A_{+o}^{(1)}\right)+i \operatorname{Tr}\left(k_{o} A_{o+}^{(1)} k_{+} A_{+o}^{(1)}\right)^{2} \\
& +i \operatorname{Tr}\left(k_{o} A_{o}^{(1)} k_{o} A_{o+}^{(1)} k_{+} A_{+}^{(1)} k_{+} A_{+o}^{(1)}\right)+i \operatorname{Tr}\left(\left(k_{o} A_{o}^{(1)}\right)^{2} k_{o} A_{o+}^{(1)} k_{+} A_{+o}^{(1)}\right) \\
& +\frac{i}{8} \operatorname{Tr}\left(k_{o} A_{o}^{(1)}\right)^{4}+O\left(V^{5}\right) .
\end{aligned}
$$

In the above formula the three first terms correspond with the one-loop contributions to the two-point functions in the inos sector; the following four terms with the contributions to the three-point functions and the last six terms are the corresponding contributions to the four-point functions.

Thus the total effective action for the two, three, and four point Green's functions is given by:

$$
\Gamma_{e f f}[V]=\Gamma_{o}[V]+\Gamma_{e f f}^{\tilde{f}}[V]+\Gamma_{e f f}^{\tilde{\chi}}[V]
$$

where $\Gamma_{o}[V]$ is the effective action at tree level and $\Gamma_{e f f}^{\tilde{f}}[V]$ and $\Gamma_{e f f}^{\tilde{\chi}}[V]$ are the effective actions generated from sfermions and inos respectively, which have been given in formulae (18) and (23).

The Feynman diagrams corresponding to the diferent terms appearing in the above equations (18) and (23) can be found in figure 1.

Finally, the effective action can be written as a function of the n-point Green's functions, $\Gamma_{\mu \nu \ldots \rho}^{V_{1} V_{2} \ldots V_{n}}$, generically as:

$$
\Gamma_{e f f}[V]=\sum_{n} \frac{1}{C_{V_{1} V_{2} \ldots V_{n}}} \int \mathrm{d} x_{1} \ldots \mathrm{d} x_{n} \Gamma_{\mu \nu \ldots \rho}^{V_{1} V_{2} \ldots V_{n}}\left(x_{1} x_{2} \ldots x_{n}\right) V_{1}^{\mu}\left(x_{1}\right) V_{2}^{\nu}\left(x_{2}\right) \ldots V_{n}^{\rho}\left(x_{n}\right)
$$


where $C_{V_{1} V_{2} \ldots V_{n}}$ are the appropriate combinatorial factors. For practical purposes, it is usefull to work in the momentum space where the effective action is given by:

$$
\Gamma_{e f f}[V]=\sum_{n} \frac{1}{C_{V_{1} V_{2} \ldots V_{n}}} \int \mathrm{d} \tilde{k}_{1} \ldots \mathrm{d} \tilde{k}_{n}(2 \pi)^{4} \delta\left(\sum_{i=1}^{n} k_{i}\right) \Gamma_{\mu \nu \ldots \rho}^{V_{1} V_{2} \ldots V_{n}}\left(k_{1} k_{2} \ldots k_{n}\right) V_{1}^{\mu}\left(k_{1}\right) V_{2}^{\nu}\left(k_{2}\right) \ldots V_{n}^{\rho}\left(k_{n}\right),
$$

where $\mathrm{d} \tilde{k} \equiv \mathrm{d}^{4} k /(2 \pi)^{4}$ and the momentum-space Green's functions $\Gamma_{\mu \nu \ldots \rho}^{V_{1} V_{2} \ldots V_{n}}\left(k_{1} k_{2} \ldots k_{n}\right)$ are the Fourier transform of the ordinary space-time Green's functions $\Gamma_{\mu \nu \ldots \rho}^{V_{1} V_{2} \ldots V_{n}}\left(x_{1} x_{2} \ldots x_{n}\right)$,

$$
(2 \pi)^{4} \delta\left(\sum_{i=1}^{n} k_{i}\right) \Gamma_{\mu \nu \ldots \rho}^{V_{1} V_{2} \ldots V_{n}}\left(k_{1}, k_{2}, \ldots, k_{n}\right) \equiv \int d x_{1} d x_{2} \ldots d x_{n} e^{i \Sigma_{i=1}^{n} k_{i} x_{i}} \Gamma_{\mu \nu \ldots \rho}^{V_{1} V_{2} \ldots V_{n}}\left(x_{1}, x_{2}, \ldots, x_{n}\right) .
$$

Our convention for the Fourier transform of the gauge bosons fields $V^{\mu}(k)$ is,

$$
V^{\mu}(k)=\int d x e^{-i k x} V^{\mu}(x) .
$$

Finally, we remind that in extracting the Green's functions from the effective action, the proper symmetrization over the indexes and momenta corresponding to the identical external fields must be performed.

\section{Decoupling in the two-point functions}

In this section and in the following we study the asymptotic behaviour of the above effective action and the corresponding Green's functions in the regime where the sparticle masses are large. By large sparticle masses limit we mean generically, $\tilde{m}_{i}^{2} \gg M_{E W}^{2}, k^{2}$, where $\tilde{m}_{i}$ denotes any of the physical sparticle masses, $M_{E W}$ any of the electroweak masses $\left(M_{Z}, M_{W}, m_{t}, \ldots\right)$ and $k$ denotes any of the external momenta. As for the analytical computation, whenever we refer to the large sparticle masses limit of a given one-loop Feynman integral, we mean the asymptotic limit $\tilde{m}_{i} \rightarrow \infty$ for all sparticle masses that are involved in that integral. However, we would like to emphasize that this asymptotic limit is not fully defined unless one specifies in addition the relative sizes of the involved masses. In other words, the result may depend, in general, on the particular way this asymptotic limit is taken. Here we consider the asymptotic limit $\tilde{m}_{i, j}^{2} \rightarrow \infty$, while keeping $\left|\frac{\tilde{m}_{i}^{2}-\tilde{m}_{j}^{2}}{\tilde{m}_{i}^{2}+\tilde{m}_{j}^{2}}\right| \ll 1$ for all $i \neq j$. That is, we consider the plaussible situation where there is a big gap between the SUSY particles and their standard partners, but the differences among the SUSY masses are not large. First, we concentrate on the two-point functions. Details of this analysis can be found in [16]. We just summarize here the main results.

By working in the momentum space and by following the standard techniques it is possible to compute the two point functios coming from the integration of the sfermions and the inos according to the discussion introduced in the previous section. The corresponding part of the effective action can be written as

$$
\Gamma_{e f f}[V]_{[2]}=\frac{1}{C_{V_{1} V_{2}}} \int \mathrm{d} \tilde{p} \mathrm{~d} \tilde{k} \delta(p+k)(2 \pi)^{4} \Gamma_{\mu \nu}^{V_{1} V_{2}}(k) V_{1}^{\mu}(p) V_{2}^{\nu}(k),
$$


where $C_{V_{1} V_{2}}=n$ and $n$ denotes the number of external gauge bosons that are identical.

The exact results for each contribution to the two-point Green's functions in momentum space and in a $R_{\xi}$ covariant gauge, $\Gamma_{\mu \nu}^{A A}(k), \Gamma_{\mu \nu}^{Z Z}(k), \Gamma_{\mu \nu}^{A Z}(k)$ and $\Gamma_{\mu \nu}^{W W}(k)$, can be found in [1]].

As it was explained in the introduction and was mentioned at the begining of this section, we are interested in the asymptotic behavior of the Green's functions for very heavy SUSY masses. Thus we need to compute not just the exact results to one-loop of the Green's functions but their asymptotic expressions valid in that limit. In order to get them we have analized the integrals by means of the so-called m-Theorem [15]. This theorem provides a powerful technique to study the asymptotic behaviour of Feynman integrals in the limit where some of the masses are large. Notice that this is non trivial since some of these integrals are divergent, in which case, the interchange of the integral with the large mass limit is not allowed. Thus, one should first compute the integrals with some regularization procedure as, for instance, dimensional regularization, and at the end take the large mass limit. Instead of this direct way it is also possible to proceed as follows: First, one rearranges the integrand through algebraic manipulations up to separate the Feynman integral into a divergent part, which can be evaluated exactly using the standard techniques of dimensional regularization, and a convergent part that satisfies the requirements demanded by the $\mathrm{m}$-Theorem and therefore, goes to zero in the infinite mass limit. By means of this procedure the correct asymptotic behaviour of the integrals is guaranteed. This is the method we will follow in this work. Some examples of the computation of the Feynman integrals by means of the mTheorem as well as details of this theorem are given in [16]. The results for the one loop integrals in the large masses limit that appear in the two-point functions are also presented in that paper.

By following the above described method we have obtained the asymptotic behaviour of the two point functions in the large sparticle masses limit, which for the sfermions and inos sectors read respectivelly as follows:

$$
\begin{aligned}
\tilde{m}_{f_{i}}^{2} & \gg M_{E W}^{2}, k^{2} ; \\
\left|\tilde{m}_{f_{i}}^{2}-\tilde{m}_{f_{j}}^{2}\right| & \ll\left|\tilde{m}_{f_{i}}^{2}+\tilde{m}_{f_{j}}^{2}\right| \forall i, j,
\end{aligned}
$$

and

$$
\begin{aligned}
\tilde{M}_{i}^{2} & \gg M_{E W}^{2}, k^{2}, \\
\left|\tilde{M}_{i}^{2}-\tilde{M}_{j}^{2}\right| & \ll\left|\tilde{M}_{i}^{2}+\tilde{M}_{j}^{2}\right| \forall i, j,
\end{aligned}
$$

where $\tilde{m}_{f_{i}}$ denotes the mass of the sfermion $\tilde{f}_{i}, \tilde{M}_{i}$ the mass of the ino $i, M_{E W}$ is any of the electroweak masses and $k$ is any of the external momenta. The results of the two-point functions $\Gamma_{\mu \nu}^{V_{1} V_{2}}(k)$ to one loop are given by,

$$
\Gamma_{\mu \nu}^{V_{1} V_{2}}=\Gamma_{0 \mu \nu}^{V_{1} V_{2}}+\Delta \Gamma_{\mu \nu}^{V_{1} V_{2}}
$$

where the tree level functions $\Gamma_{0 \mu \nu}^{V_{1} V_{2}}$ in a $R_{\xi}$ covariant gauge are,

$$
\Gamma_{0 \mu \nu}^{V V}(k)=\left(M_{V}^{2}-k^{2}\right) g_{\mu \nu}+\left(1-\frac{1}{\xi_{V}}\right) k_{\mu} k_{\nu}(V=Z, W),
$$




$$
\Gamma_{0 \mu \nu}^{A A}=-k^{2} g_{\mu \nu}+\left(1-\frac{1}{\xi_{A}}\right) k_{\mu} k_{\nu}, \quad \Gamma_{0 \mu \nu}^{V_{1} V_{2}}=0 \text { if } V_{1} \neq V_{2}
$$

and the contributions from sfermions and inos, $\Delta \Gamma_{\mu \nu}^{V_{1} V_{2}}$, can be written as,

$$
\Delta \Gamma_{\mu \nu}^{V_{1} V_{2}}(k)=\Sigma^{V_{1} V_{2}}(k) g_{\mu \nu}+R^{V_{1} V_{2}}(k) k_{\mu} k_{\nu}
$$

We have shown in [16] that the asymptotic results are of the generic form:

$$
\begin{aligned}
& \Sigma^{V_{1} V_{2}}(k)=\Sigma_{(0)}^{V_{1} V_{2}}+\Sigma_{(1)}^{V_{1} V_{2}} k^{2}+O\left(\frac{k^{2}}{\Sigma \tilde{m}^{2}}, \frac{\triangle \tilde{m}^{2}}{\Sigma \tilde{m}^{2}}\right), \\
& R^{V_{1} V_{2}}(k)=R_{(0)}^{V_{1} V_{2}}+O\left(\frac{k^{2}}{\Sigma \tilde{m}^{2}}, \frac{\triangle \tilde{m}^{2}}{\Sigma \tilde{m}^{2}}\right),
\end{aligned}
$$

where $\Sigma_{(1)}^{V_{1} V_{2}}$ and $R_{(0)}^{V_{1} V_{2}}$ contain the divergent contributions, namely the $O(1 / \epsilon)$ terms in dimensional regularization and are functions of the large SUSY masses but are $k$ independent. Furthermore, we find $R_{(0)}^{V_{1} V_{2}}=-\Sigma_{(1)}^{V_{1} V_{2}}$ in this asymptotic regime. On the other hand, the $\Sigma_{(0)}^{V_{1} V_{2}}$ functions turn out to be finite and $k$ independent, and they vanish in the asymptotic limit of infinite sparticle masses. Here and in the following the terms denoted by $O\left(\frac{k^{2}}{\Sigma \tilde{m}^{2}}, \frac{\Delta \tilde{m}^{2}}{\Sigma \tilde{m}^{2}}\right)$ are suppressed by inverse powers of the large SUSY masses and vanish in the asymptotic regime. The large mass parameter of the asymptotic expansion in the two-point functions is always taken to be the sum of the various squared masses involved in the corresponding loop diagram which we denote here generically by $\Sigma \tilde{m}^{2}$. On the other hand, $\triangle \tilde{m}^{2}$ represents the various corresponding squared mass differences which in our asymptotic limit are always smaller than the corresponding sum.

These results can alternatively be expressed through the tranverse and longitudinal parts of the two-point functions, $\Sigma_{T}^{V_{1} V_{2}}$ and $\Sigma_{L}^{V_{1} V_{2}}$, which are defined by,

$$
\Gamma_{\mu \nu}^{V_{1} V_{2}}(k)=\Gamma_{0 \mu \nu}^{V_{1} V_{2}}(k)+\Sigma_{T}^{V_{1} V_{2}}(k)\left(g_{\mu \nu}-\frac{k_{\mu} k_{\nu}}{k^{2}}\right)+\Sigma_{L}^{V_{1} V_{2}}(k) \frac{k_{\mu} k_{\nu}}{k^{2}} .
$$

According to this definition, the asymptotic results whose explicit expressions are given in 16 can be written, in a generic form, as:

$$
\begin{aligned}
& \Sigma_{T}^{V_{1} V_{2}}(k)=\Sigma_{(0)}^{V_{1} V_{2}}+\Sigma_{(1)}^{V_{1} V_{2}} k^{2}+O\left(\frac{k^{2}}{\Sigma \tilde{m}^{2}}, \frac{\triangle \tilde{m}^{2}}{\Sigma \tilde{m}^{2}}\right), \\
& \Sigma_{L}^{V_{1} V_{2}}(k)=\Sigma_{(0)}^{V_{1} V_{2}}+O\left(\frac{k^{2}}{\Sigma \tilde{m}^{2}}, \frac{\triangle \tilde{m}^{2}}{\Sigma \tilde{m}^{2}}\right),
\end{aligned}
$$

Notice that $\left(\Sigma_{L}^{V_{1} V_{2}}-\Sigma_{T}^{V_{1} V_{2}}\right) \propto k^{2}$. This result together with the explicit form of the $\Sigma_{(0)}^{V_{1} V_{2}}$ and $\Sigma_{(1)}^{V_{1} V_{2}}$ functions demostrate that the decoupling indeed occurs in the two-point functions. 
In order to illustrate the above result with one particular example, we choose to present here the explicit expressions for the $\Sigma^{Z Z}$ contributions. The transverse contributions are [16]:

$$
\begin{aligned}
& \Sigma_{T}^{Z Z}(k)_{\tilde{q}}=N_{c} \frac{e^{2}}{16 \pi^{2}} \frac{1}{s_{W}^{2} c_{W}^{2}} \sum_{\tilde{q}}\left\{\frac{1}{2}\left[c_{t}^{2} s_{t}^{2} h\left(\tilde{m}_{t_{1}}^{2}, \tilde{m}_{t_{2}}^{2}\right)+c_{b}^{2} s_{b}^{2} h\left(\tilde{m}_{b_{1}}^{2}, \tilde{m}_{b_{2}}^{2}\right)\right]\right. \\
& -\frac{1}{3} k^{2}\left[\left(\frac{c_{t}^{2}}{2}-\frac{2 s_{W}^{2}}{3}\right)^{2}\left(\Delta_{\epsilon}-\log \frac{\tilde{m}_{t_{1}}^{2}}{\mu_{o}^{2}}\right)+\left(\frac{s_{t}^{2}}{2}-\frac{2 s_{W}^{2}}{3}\right)^{2}\left(\Delta_{\epsilon}-\log \frac{\tilde{m}_{t_{2}}^{2}}{\mu_{o}^{2}}\right)\right. \\
& +\left(-\frac{c_{b}^{2}}{2}+\frac{s_{W}^{2}}{3}\right)^{2}\left(\Delta_{\epsilon}-\log \frac{\tilde{m}_{b_{1}}^{2}}{\mu_{o}^{2}}\right)+\left(-\frac{s_{b}^{2}}{2}+\frac{s_{W}^{2}}{3}\right)^{2}\left(\Delta_{\epsilon}-\log \frac{\tilde{m}_{b_{2}}^{2}}{\mu_{o}^{2}}\right) \\
& \left.\left.+\frac{1}{2} s_{t}^{2} c_{t}^{2}\left(\Delta_{\epsilon}-\log \frac{\tilde{m}_{t_{1}}^{2}+\tilde{m}_{t_{2}}^{2}}{2 \mu_{o}^{2}}\right)+\frac{1}{2} s_{b}^{2} c_{b}^{2}\left(\Delta_{\epsilon}-\log \frac{\tilde{m}_{b_{1}}^{2}+\tilde{m}_{b_{2}}^{2}}{2 \mu_{o}^{2}}\right)\right]\right\} \text {, } \\
& \Sigma_{T}^{Z Z}(k)_{\tilde{l}}=-\frac{e^{2}}{16 \pi^{2}} \frac{1}{s_{W}^{2} c_{W}^{2}} \sum_{\tilde{l}}\left\{-\frac{1}{2} c_{\tau}^{2} s_{\tau}^{2} h\left(\tilde{m}_{\tau_{1}}^{2}, \tilde{m}_{\tau_{2}}^{2}\right)+\frac{1}{3} k^{2}\left[\frac{1}{4}\left(\Delta_{\epsilon}-\log \frac{\tilde{m}_{\nu}^{2}}{\mu_{o}^{2}}\right)\right.\right. \\
& +\left(\frac{-c_{\tau}^{2}}{2}+s_{W}^{2}\right)^{2}\left(\Delta_{\epsilon}-\log \frac{\tilde{m}_{\tau_{1}}^{2}}{\mu_{o}^{2}}\right)+\left(-\frac{s_{\tau}^{2}}{2}+s_{W}^{2}\right)^{2}\left(\Delta_{\epsilon}-\log \frac{\tilde{m}_{\tau_{2}}^{2}}{\mu_{o}^{2}}\right) \\
& \left.\left.+\frac{1}{2} s_{\tau}^{2} c_{\tau}^{2}\left(\Delta_{\epsilon}-\log \frac{\tilde{m}_{\tau_{1}}^{2}+\tilde{m}_{\tau_{2}}^{2}}{2 \mu_{o}^{2}}\right)\right]\right\} \text {, } \\
& \Sigma_{T}^{Z Z}(k)_{\tilde{\chi}}=-\frac{e^{2}}{16 \pi^{2}} \frac{1}{s_{W}^{2} c_{W}^{2}}\left\{-\frac{1}{2}\left(\tilde{M}_{3}^{o}-\tilde{M}_{4}^{o}\right)^{2}\left(\Delta_{\epsilon}-\log \frac{\tilde{M}_{3}^{o 2}+\tilde{M}_{4}^{o 2}}{2 \mu_{o}^{2}}\right)\right. \\
& +\frac{1}{3} k^{2}\left[4\left(s_{W}^{2}-1\right)^{2}\left(\Delta_{\epsilon}-\log \frac{\tilde{M}_{1}^{+2}}{\mu_{o}^{2}}\right)+4\left(s_{W}^{2}-\frac{1}{2}\right)^{2}\left(\Delta_{\epsilon}-\log \frac{\tilde{M}_{2}^{+2}}{\mu_{o}^{2}}\right)\right. \\
& \left.\left.+\left(\Delta_{\epsilon}-\log \frac{\tilde{M}_{3}^{o 2}+\tilde{M}_{4}^{o 2}}{2 \mu_{o}^{2}}\right)\right]\right\}
\end{aligned}
$$

The results for the corresponding longitudinal parts can, generically, be written as:

$$
\Sigma_{L}^{Z Z}(k)=\left[\text { Term in } \Sigma_{T}^{Z Z}(k) \text { that is } k \text { independent }\right] \equiv \Sigma_{(0)}^{Z Z} .
$$

In the above equations $c_{f}=\cos \theta_{f}, s_{f}=\sin \theta_{f}$, with $\theta_{f}$ being the mixing angle in the $f$-sector, and the sum in $\tilde{q}$ and $\tilde{l}$ run over the three squarks and sleptons generations respectively. Besides,

$$
\Delta_{\epsilon}=\frac{2}{\epsilon}-\gamma_{\epsilon}+\log (4 \pi), \epsilon=4-D ;
$$

$\mu_{o}$ is the usual mass scale appearing in dimensional regularization and the function $h\left(m_{1}^{2}, m_{2}^{2}\right)$ is given by,

$$
h\left(m_{1}^{2}, m_{2}^{2}\right) \equiv m_{1}^{2} \log \frac{2 m_{1}^{2}}{m_{1}^{2}+m_{2}^{2}}+m_{2}^{2} \log \frac{2 m_{2}^{2}}{m_{1}^{2}+m_{2}^{2}},
$$

which behaves as:

$$
h\left(m_{1}^{2}, m_{2}^{2}\right) \rightarrow \frac{m_{1}^{2}-m_{2}^{2}}{2}\left[\frac{\left(m_{1}^{2}-m_{2}^{2}\right)}{\left(m_{1}^{2}+m_{2}^{2}\right)}+O\left(\frac{m_{1}^{2}-m_{2}^{2}}{m_{1}^{2}+m_{2}^{2}}\right)^{2}\right]
$$


in the asymptotic limit. The explicit expressions for the other two-point functions, $\Gamma^{A A}, \Gamma^{A Z}$, and $\Gamma^{W W}$ can be found in [16].

As can be seen from our total results [16], all the non-vanishing contributions to the twopoint functions in the asymptotic region are contained in $\Sigma_{(1)}^{V_{1} V_{2}}$ and $R_{(0)}^{V_{1} V_{2}}$ and, besides, $R_{(0)}^{V_{1} V_{2}}=$ $-\Sigma_{(1)}^{V_{1} V_{2}}$. Therefore, they can be absorbed into a redefinition of the SM relevant parameters, $M_{W}, M_{Z}$ and $e$ and the gauge bosons wave functions. In consequence, the decoupling of squarks, sleptons, charginos and neutralinos in the two point functions do indeed occur.

\section{The three-point functions}

In this section we present the three-point functions for the electroweak gauge bosons to one loop and analize the large masses limit of the SUSY particles.

In order to get the explicit expressions for the these functions one must work out the corresponding functional traces in the formulae (18) and (23). For this purpose one must substitute all the operators and propagators in these formulae, and compute all the appearing Dirac traces. The functional traces also involve to perform the sum in the corresponding matrix indexes, the sum over the various types of sfermions and the sum in color indexes in the case of squarks. We would like to mention that, in this paper, we have chosen to work in the momentum space, which turns out to simplify considerably the calculation of the functional traces.

By following the same procedure as in section 3 we have obtained the result for the effective action of the three-point functions coming from the integration of sfermions and inos. Generically, the corresponding part of the effective action can be written as,

$$
\Gamma_{e f f}[V]_{[3]}=\frac{1}{C_{V_{1} V_{2} V_{3}}} \int \mathrm{d} \tilde{p} \mathrm{~d} \tilde{k} \mathrm{~d} \tilde{r} \delta(p+k+r)(2 \pi)^{4} \Gamma_{\mu \nu \sigma}^{V_{1} V_{2} V_{3}} V_{1}^{\mu}(p) V_{2}^{\nu}(k) V_{3}^{\sigma}(r),
$$

where $C_{V_{1} V_{2} V_{3}}=n$ ! and $n$ is the number of external gauge bosons that are identical.

\subsection{Sfermions contributions}

For simplicity, we show here the results in a general and compact form and leave the details for the appendices. Once the appropiate traces have been computed, the corresponding effective action for the three-point functions coming from the sfermions integration can be expressed as,

$$
\begin{aligned}
\Gamma_{e f f}^{\tilde{f}}[V]_{[3]} & =-\pi^{2} \int \mathrm{d} \tilde{p} \mathrm{~d} \tilde{k} \mathrm{~d} \tilde{r} \delta(p+k+r) \sum_{\tilde{f}}\left(\sum_{a, b}\left(\hat{O}^{1 \mu}\right)_{a b}\left(\hat{O}^{2 \nu \sigma}\right)_{b a} T_{\mu}^{a b}\left(p, \tilde{m}_{f_{a}}, \tilde{m}_{f_{b}}\right) g_{\nu \sigma}\right. \\
& \left.-\frac{1}{3} \sum_{a, b, c}\left(\hat{O}^{1 \mu}\right)_{a b}\left(\hat{O}^{1 \nu}\right)_{b c}\left(\hat{O}^{1 \sigma}\right)_{c a} T_{\mu \nu \sigma}^{a b c}\left(p, k, \tilde{m}_{f_{a}}, \tilde{m}_{f_{b}}, \tilde{m}_{f_{c}}\right)\right),
\end{aligned}
$$


where, similarly to the two-point functions, the sum in $\tilde{f}$ runs over the three generations and over the $N_{c}$ colors in the case of squarks, the indexes $a, b$ and $c$ run from one to four corresponding to the four entries of the sfermions column matrix $\tilde{f} . T_{\mu}^{a b}$ and $T_{\mu \nu \sigma}^{a b c}$ are the one-loop integrals that are defined as functions of the standard integrals in Appendix A, and $\hat{O}^{1 \mu}$ and $\hat{O}^{2 \mu \nu}$ are the operators collected in Appendix B. It is important to emphasize that this formula is exact to one loop.

By substituting the definition of the operators involved in the above equation, we have obtained all the contributions to the 3-point functions to one loop. In particular, the exact results for $A W^{+} W^{-}$and $Z W^{+} W^{-}$are given in Appendix C.

Futhermore, as we are interested in the large mass limit of the SUSY particles, we need the asymptotic expressions for the integrals appearing in the formula (44), which we have obtained by means of the m-Theorem. The results of the these integrals in that limit can be easily read from eqs. (A.3) and (A.4) respectively and by using the corresponding asymptotic expressions for the scalar and tensor integrals that have been presented in Appendix A. By substituting these asymptotic results into eq. (44) we finally get,

$$
\begin{aligned}
\Gamma_{e f f}^{\tilde{f}}[V]_{[3]}= & \frac{\pi^{2}}{9} \int \mathrm{d} \tilde{p} \mathrm{~d} \tilde{k} \mathrm{~d} \tilde{r} \delta(p+k+r) \sum_{\tilde{f}} \\
& \left\{\sum_{a, b, c}\left(\hat{O}^{1 \mu}\right)_{a b}\left(\hat{O}^{1 \nu}\right)_{b c}\left(\hat{O}^{1 \sigma}\right)_{c a}\left(\Delta_{\epsilon}-\log \frac{\tilde{m}_{f a}^{2}+\tilde{m}_{f b}^{2}+\tilde{m}_{f c}^{2}}{3 \mu_{o}^{2}}\right) \mathrm{E}_{\mu \nu \sigma}\right\},
\end{aligned}
$$

where $\mathrm{E}_{\mu \nu \sigma}$ denotes the tensor appearing in the tree level vertex defined by,

$$
\mathrm{E}_{\mu \nu \sigma} \equiv\left[(k-p)_{\sigma} g_{\mu \nu}+(r-k)_{\mu} g_{\nu \sigma}+(p-r)_{\nu} g_{\mu \sigma}\right] \text {. }
$$

Therefore, the asymptotic result in eq. (45) is proportional to the tree level tensor $\mathrm{E}_{\mu \nu \sigma}$. Thus, we can already conclude at this point that the sfermions decouple in the three-point functions since this correction being proportional to $\mathrm{E}_{\mu \nu \sigma}$ can be absorbed into redefinitions of the SM parameters and the external gauge bosons wave functions. Notice that the two kind of one-loop Feynman integrals that appear in the three-point functions, $T_{\mu}^{a b}$ and $T_{\mu \nu \sigma}^{a b c}$, involve generically two and three different sparticle masses respectively, which in our limit are considered to be large. However, in order to implement the large SUSY masses limit one must choose a proper combination of masses such that there is just one large mass parameter while the others are kept small. Our choice for the large mass parameter is always the sum of the various squared SUSY masses involved in the loop integral. The rest of the mass parameters can be expressed in terms of the sparticle squared mass differences which in our approximation are small as compared to their sum. The result in eq. (45) has corrections not explicitely shown which are suppresed by inverse powers of these large SUSY mass sums and therefore they vanish in our asymptotic limit. For completeness, we present also here the explicit contributions to the three-point Green's functions with specific external gauge bosons, $\Gamma_{\mu \nu \sigma}^{V_{1} V_{2} V_{3}}$. Our results are presented in the form:

$$
\Gamma_{\mu \nu \sigma}^{V_{1} V_{2} V_{3}}=\Gamma_{0 \mu \nu \sigma}^{V_{1} V_{2} V_{3}}+\Delta \Gamma_{\mu \nu \sigma}^{V_{1} V_{2} V_{3}}
$$


where the outgoing momenta assignements are $V_{1}^{\mu}(p), V_{2}^{\nu}(k)$ and $V_{3}^{\sigma}(r)$ and the tree level contributions are:

$$
\Gamma_{0 \mu \nu \sigma}^{A W^{+} W^{-}}=-e \mathrm{E}_{\mu \nu \sigma}, \Gamma_{0 \mu \nu \sigma}^{Z W^{+} W^{-}}=-g c_{W} \mathrm{E}_{\mu \nu \sigma} .
$$

In order to get the sfermions contributions, one must substitute all the operators that appear in eq.(45), perform the corresponding sums and after a rather lengthy calculation, the following results are obtained:

$$
\begin{aligned}
\Delta \Gamma_{\mu \nu \sigma}^{A W^{+} W^{-}} & =\frac{e g^{2}}{16 \pi^{2}} \frac{N_{c}}{9} \mathrm{Ł}_{\mu \nu \sigma} \sum_{\tilde{q}} \frac{1}{2}\left\{\Delta_{\epsilon}+f_{1}\left(\tilde{m}_{t_{1}}^{2}, \tilde{m}_{t_{2}}^{2}, \tilde{m}_{b_{1}}^{2}, \tilde{m}_{b_{2}}^{2}\right)\right\} \\
& +F_{1 \mu \nu \sigma}\left[O\left(\frac{p^{2}}{\Sigma \tilde{m}^{2}}, \frac{\triangle \tilde{m}^{2}}{\Sigma \tilde{m}^{2}}\right)\right], \\
\Delta \Gamma_{\mu \nu \sigma}^{Z W^{+} W_{\tilde{q}}^{-}} & =-\frac{g^{3}}{16 \pi^{2}} \frac{N_{c}}{6 c_{W}} \mathrm{Ł}_{\mu \nu \sigma} \sum_{\tilde{q}} \frac{1}{3} s_{W}^{2}\left\{\Delta_{\epsilon}+f_{2}\left(\tilde{m}_{t_{1}}^{2}, \tilde{m}_{t_{2}}^{2}, \tilde{m}_{b_{1}}^{2}, \tilde{m}_{b_{2}}^{2}\right)\right\} \\
& +F_{2 \mu \nu \sigma}\left[O\left(\frac{p^{2}}{\Sigma \tilde{m}^{2}}, \frac{\triangle \tilde{m}^{2}}{\Sigma \tilde{m}^{2}}\right)\right],
\end{aligned}
$$

where, generically, $p$ denotes any of the external momenta, $\triangle \tilde{m}^{2}$ denotes the various squared mass differences and $\Sigma \tilde{m}^{2}$ denotes the corresponding large mass parameter which in our case is always a sum of squared SUSY masses. The functions $F_{i \mu \nu \sigma}(i=1,2)$ are finite and they go to zero in the limit of $\tilde{m}_{i, j} \rightarrow \infty(\forall i, j)$ with $\left|\tilde{m}_{i}^{2}-\tilde{m}_{j}^{2}\right| \ll\left|\tilde{m}_{i}^{2}+\tilde{m}_{j}^{2}\right|$. The functions $f_{1,2}\left(\tilde{m}_{t_{1}}^{2}, \tilde{m}_{t_{2}}^{2}, \tilde{m}_{b_{1}}^{2}, \tilde{m}_{b_{2}}^{2}\right)$ are given explicitely in Appendix B. These functions are also finite but different from zero in the large masses limit and therefore, they contain all the potentially non-decoupling effects of the three-point functions. More specifically, these effects are given by the logarithmic dependence on the large mass parameter of these two functions. Generically, these can be written as,

$$
f_{1,2}\left(\tilde{m}_{t_{1}}^{2}, \tilde{m}_{t_{2}}^{2}, \tilde{m}_{b_{1}}^{2}, \tilde{m}_{b_{2}}^{2}\right)=O\left(\log \frac{\Sigma \tilde{m}^{2}}{\mu_{o}^{2}}\right)+O\left(\frac{\triangle \tilde{m}^{2}}{\Sigma \tilde{m}^{2}}\right)
$$

As we have mentioned before, the corrections $\Delta \Gamma$ are proportional to the tree level vertex $\mathrm{E}_{\mu \nu \sigma}$, and therefore the potentially non-decoupling effects in the three-point functions can be absorbed into redefinitions of the coupling constants and wave functions renormalization. Therefore, this is an explicit proof of decoupling of squarks in the $\Gamma_{\mu \nu \sigma}^{A W^{+} W^{-}}$and $\Gamma_{\mu \nu \sigma}^{Z W^{+} W^{-}}$Green functions.

We would like to point out that the other three-point Green's functions are exactly zero in our limit, as it was expected. As a check of the previous functional computation we have also calculated all these three point functions by diagramatic methods and we have got the same results.

Similar results are obtained for the sleptons sector doing the corresponding replacements: $\tilde{q} \rightarrow \tilde{l}, N_{c} \rightarrow 1, \tilde{m}_{t_{1}} \rightarrow \tilde{m}_{\nu}, \tilde{m}_{b_{1}} \rightarrow \tilde{m}_{\tau_{1}}, \tilde{m}_{b_{2}} \rightarrow \tilde{m}_{\tau_{2}}, c_{t} \rightarrow 1, s_{t} \rightarrow 0, c_{b} \rightarrow c_{\tau}, s_{b} \rightarrow s_{\tau}$ and $y_{f}=\frac{1}{3} \rightarrow y_{f}=-1$. 


\subsection{Inos contributions}

To compute the inos contributions to the three-point functions one must work out the functional traces given in eq. (23). This leads to an expression containing several combinations of momenta, operators and Dirac traces corresponding to specific external gauge bosons $V_{1} V_{2} V_{3}$ that we give explicitely in Appendix B.

The result for the effective action coming from the integration of inos in the three-point functions can be expressed in a compact form as,

$$
\begin{aligned}
& \Gamma_{e f f}^{\tilde{\chi}}[V]_{[3]}=-i \int \mathrm{d} \tilde{p} \mathrm{~d} \tilde{k} \mathrm{~d} \tilde{r}(2 \pi)^{4} \delta(p+k+r) \times \\
& \int d \widehat{q}\left[\frac { 1 } { 3 } \sum _ { i , j , k = 1 } ^ { 2 } \mathcal { F } ^ { i j k } ( \tilde { M } _ { i } ^ { + } , \tilde { M } _ { j } ^ { + } , \tilde { M } _ { k } ^ { + } ) \left\{q_{1}^{\alpha} q_{2}^{\beta} q_{3}^{\gamma}(G \cdot O)_{123}^{+++}\right.\right. \\
& \left.+q_{1}^{\alpha} \tilde{M}_{j}^{+} \tilde{M}_{k}^{+}(G \cdot O)_{1}^{+++}+q_{2}^{\alpha} \tilde{M}_{i}^{+} \tilde{M}_{k}^{+}(G \cdot O)_{2}^{+++}+q_{3}^{\alpha} \tilde{M}_{i}^{+} \tilde{M}_{j}^{+}(G \cdot O)_{3}^{+++}\right\} \\
& +\sum_{i=1}^{4} \sum_{j, k=1}^{2} \mathcal{F}^{i j k}\left(\tilde{M}_{i}^{o}, \tilde{M}_{j}^{+}, \tilde{M}_{k}^{+}\right)\left\{q_{1}^{\alpha} q_{2}^{\beta} q_{3}^{\gamma}(G \cdot O)_{123}^{o++}\right. \\
& \left.+q_{1}^{\alpha} \tilde{M}_{j}^{+} \tilde{M}_{k}^{+}(G \cdot O)_{1}^{o++}+q_{2}^{\alpha} \tilde{M}_{i}^{o} \tilde{M}_{k}^{+}(G \cdot O)_{2}^{o++}+q_{3}^{\alpha} \tilde{M}_{i}^{o} \tilde{M}_{j}^{+}(G \cdot O)_{3}^{o++}\right\} \\
& +\sum_{i, j=1}^{4} \sum_{k=1}^{2} \mathcal{F}^{i j k}\left(\tilde{M}_{i}^{o}, \tilde{M}_{j}^{o}, \tilde{M}_{k}^{+}\right)\left\{q_{1}^{\alpha} q_{2}^{\beta} q_{3}^{\gamma}(G \cdot O)_{123}^{o o+}\right. \\
& \left.+q_{1}^{\alpha} \tilde{M}_{j}^{o} \tilde{M}_{k}^{+}(G \cdot O)_{1}^{o o+}+q_{2}^{\alpha} \tilde{M}_{i}^{o} \tilde{M}_{k}^{+}(G \cdot O)_{2}^{o o+}+q_{3}^{\alpha} \tilde{M}_{i}^{o} \tilde{M}_{j}^{o}(G \cdot O)_{3}^{o o+}\right\} \\
& +\frac{1}{6} \sum_{i, j, k=1}^{4} \mathcal{F}^{i j k}\left(\tilde{M}_{i}^{o}, \tilde{M}_{j}^{o}, \tilde{M}_{k}^{o}\right)\left\{q_{1}^{\alpha} q_{2}^{\beta} q_{3}^{\gamma}(G \cdot O)_{123}^{o o o}\right. \\
& \left.\left.+q_{1}^{\alpha} \tilde{M}_{j}^{o} \tilde{M}_{k}^{o}(G \cdot O)_{1}^{o o o}+q_{2}^{\alpha} \tilde{M}_{i}^{o} \tilde{M}_{k}^{o}(G \cdot O)_{2}^{o o o}+q_{3}^{\alpha} \tilde{M}_{i}^{o} \tilde{M}_{j}^{o}(G \cdot O)_{3}^{o o o}\right\}\right]
\end{aligned}
$$

where $(G \cdot O)$ denotes the various products of traces and operators that are collected in Appendix B. The superscripts in $(G \cdot O)$ correspond with the type of sparticles appearing in the loop or, equivalently, in the internal Feynman's propagators, and the subscripts denote the corresponding momenta to be contracted with the results of the traces in each case. For example, in $(G \cdot O)_{123}^{+++}$, the superscripts +++ denote the three charginos in the loop and the subscripts 123 mean that

the traces must be contracted with the $q_{1}, q_{2}$ and $q_{3}$ momenta. The indexes $i, j, k$ in the above formula vary as $i, j, k=1,2$ if they refer to charginos and as $i, j, k=1, \ldots, 4$ if they refer to neutralinos and the generic function $\mathcal{F}^{i j k}\left(\tilde{M}_{i}, \tilde{M}_{j}, \tilde{M}_{k}\right)$ is given by,

$$
\mathcal{F}^{i j k}\left(\tilde{M}_{i}, \tilde{M}_{j}, \tilde{M}_{k}\right)=\frac{1}{\left[q_{1}^{2}-\tilde{M}_{i}^{2}\right]\left[q_{2}^{2}-\tilde{M}_{j}^{2}\right]\left[q_{3}{ }^{2}-\tilde{M}_{k}^{2}\right]},
$$

where,

$$
q_{1} \equiv q, q_{2} \equiv q+p \quad, q_{3} \equiv q+p+k .
$$


As we have explained above, the next step is to compute each Dirac trace appearing in the expression (52), substitute the operators, perform the corresponding traces and finally to extract the various three-point functions with specific external legs which we do not present entirely here for brevity. We have computed each contribution to these functions and have checked that the results for $\Delta \Gamma^{A A A}, \Delta \Gamma^{A A Z}, \Delta \Gamma^{A Z Z}$ and $\Delta \Gamma^{Z Z Z}$ are finite as it was expected.

The exact results to one loop for the $A W^{+} W^{-}$and $Z W^{+} W^{-}$three point functions are collected in Appendix C.

In order to get the assymptotic limit of the Green's functions in eqs. (52), (C.7) and (C.8) we use the results of the one loop integrals in the large masses limit that are collected in Appendix A and the values for the coupling matrices $O_{L, R}, O_{L, R}^{\prime}$ and $O_{L, R}^{\prime \prime}$ in the limit of large neutralino and chargino masses that can be found in [16]. By substituting all these results into eq. (52) we find the inos contributions to the three-point part of the effective action which can be written as:

$$
\begin{aligned}
& \Gamma_{e f f}^{\tilde{\chi}}[V]_{[3]}=-\frac{4}{3} \pi^{2} \int \mathrm{d} \tilde{p} \mathrm{~d} \tilde{k} \mathrm{~d} \tilde{r} \delta(p+k+r) \sum_{i, j, k}\left\{\frac{1}{3}\left(\hat{O}^{1}+\hat{O}^{2}+\hat{O}^{4}+\hat{O}^{6}+\hat{O}^{8}\right)_{i j k}^{\mu \nu \sigma}\right. \\
& \left.+\frac{1}{6} \hat{O}_{i j k}^{12 \mu \nu \sigma}+\left(\hat{O}^{16}+\hat{O}^{18}\right)_{i j k}^{\mu \nu \sigma}+\hat{O}_{i j k}^{22 \mu \nu \sigma}\right\}\left(\Delta_{\epsilon}-\log \frac{\tilde{M}_{i}^{2}+\tilde{M}_{j}^{2}+\tilde{M}_{k}^{2}}{3 \mu_{o}^{2}}\right) \mathrm{E}_{\mu \nu \sigma},
\end{aligned}
$$

where $\mathrm{E}_{\mu \nu \sigma}$ represents the tree level tensor defined in eq. (46) and the operators $\hat{O}_{i j k}^{\mu \nu \sigma}$ can be found in Appendix B. Notice that the indices $i j k$ vary in accordance with the inos particles appearing in the loops, i.e, $i, j, k=1,2$ if they refer to charginos and $i, j, k=1, \ldots, 4$ if they refer to neutralinos.

The fact that this result is proportional again to the tree level tensor $\mathrm{E}_{\mu \nu \sigma}$ enables us to conclude that the inos also decouple in the three-point functions. For completeness we have worked out, in detail, the explicit expressions for the three-point functions with specific external gauge bosons that are different from zero in our limit. By using the same notation as in subsection 4.1 we have obtained,

$$
\begin{aligned}
\Delta \Gamma_{\mu \nu \sigma}^{A W^{+} W^{-}} & =\frac{e g^{2}}{16 \pi^{2}} \frac{4}{3} \mathrm{E}_{\mu \nu \sigma}\left\{\frac{3}{2} \Delta_{\epsilon}+f_{3}\left(\tilde{M}_{1}^{+}, \tilde{M}_{2}^{+}, \tilde{M}_{1}^{0}, \tilde{M}_{2}^{0}, \tilde{M}_{3}^{0}, \tilde{M}_{4}^{0}\right)\right\} \\
& +F_{3 \mu \nu \sigma}\left[O\left(\frac{p^{2}}{\Sigma \tilde{m}^{2}}, \frac{\triangle \tilde{m}^{2}}{\Sigma \tilde{m}^{2}}\right)\right], \\
\Delta \Gamma_{\mu \nu \sigma}^{Z W^{+} W^{-}} & =\frac{g^{3}}{16 \pi^{2}} \frac{4}{3 c_{W}} \mathrm{E}_{\mu \nu \sigma}\left\{\frac{3}{2} c_{W}^{2} \Delta_{\epsilon}+f_{4}\left(\tilde{M}_{1}^{+}, \tilde{M}_{2}^{+}, \tilde{M}_{1}^{0}, \tilde{M}_{2}^{0}, \tilde{M}_{3}^{0}, \tilde{M}_{4}^{0}\right)\right\} \\
& +F_{4 \mu \nu \sigma}\left[O\left(\frac{p^{2}}{\Sigma \tilde{m}^{2}}, \frac{\triangle \tilde{m}^{2}}{\Sigma \tilde{m}^{2}}\right)\right],
\end{aligned}
$$

where the functions $F_{i \mu \nu \sigma}(i=3,4)$ are finite and we have proved explicitly that they go to zero in our asymptotic limit. On the other hand, the functions $f_{i}\left(\tilde{M}_{1}^{+}, \tilde{M}_{2}^{+}, \tilde{M}_{1}^{0}, \tilde{M}_{2}^{0}, \tilde{M}_{3}^{0}, \tilde{M}_{4}^{0}\right)(i=3,4)$ are finite and different from zero in the large masses limit and therefore, they contain all the potentially non-decoupling effects of the three-point functions. Their explicit expressions can be 
found in Appendix B. However, as we have commented above, the corrections $\Delta \Gamma$ given in (55) and (56), are also proportional to the tree level tensor $\mathrm{E}_{\mu \nu \sigma}$ and therefore, the mentioned potentially non-decoupling effects can be absorbed into redefenitions of the SM parameters and the gauge bosons wave functions. The results in eqs. (55) and (56) demostrate explicitely, therefore, the decoupling of inos in the $\Gamma^{A W^{+} W^{-}}$and $\Gamma^{Z W^{+} W^{-}}$functions.

In addition, we have checked that after the proper symmetrization over the identical external fields, the $\Delta \Gamma^{A A A}, \Delta \Gamma^{A A Z}, \Delta \Gamma^{A Z Z}$ and $\Delta \Gamma^{Z Z Z}$ contributions are exactly zero in our limit as it was expected since there are no corresponding tree level vertices. As a check of the previous functional computation we have also calculated all these three point functions by diagramatic methods and we have got the same results.

\section{The four-point functions and higher}

In this section we compute the four-point Green's functions with external gauge bosons, $A, Z, W^{+}, W^{-}$, at one loop level. At the end of this section we discuss also the case of higher point functions, which completes our analysis of decoupling of SUSY particles.

Let us begin by writting the expression of the corresponding part of the effective action as a function of the 4-point functions $\Gamma_{\mu \nu \sigma \lambda}^{V_{1} V_{2} V_{3} V_{4}}$,

$$
\Gamma_{e f f}[V]_{[4]}=\frac{1}{C_{V_{1} V_{2} V_{3} V_{4}}} \int \mathrm{d} \tilde{p} \mathrm{~d} \tilde{k} \mathrm{~d} \tilde{r} \mathrm{~d} \tilde{t} \delta(p+k+r+t)(2 \pi)^{4} \Gamma_{\mu \nu \sigma \lambda}^{V_{1} V_{2} V_{3} V_{4}} V_{1}^{\mu}(p) V_{2}^{\nu}(k) V_{3}^{\sigma}(r) V_{4}^{\lambda}(t),
$$

where $C_{V_{1} V_{2} V_{3} V_{4}}$ is the appropriate combinatorial factor for the number of identical external gauge bosons.

By working in the momentum space and by following the same techniques described in the previous sections one computes the four-point functions coming from the integrations of sfermions and inos. Clearly, this computation involves to work out again the corresponding functional traces given in eqs. (18) and (23).

\subsection{Sfermions contributions}

The resulting effective action for 4-point functions that are generated from sfermions integration can be summarized in the following expression,

$$
\begin{aligned}
\Gamma_{e f f}^{\tilde{f}}[V]_{[4]}= & \pi^{2} \int \mathrm{d} \tilde{p} \mathrm{~d} \tilde{k} \mathrm{~d} \tilde{r} \mathrm{~d} \tilde{t} \delta(p+k+r+t) \sum_{\tilde{f}} \times \\
& \left(\frac{1}{2} \sum_{a, b}\left(\hat{O}^{2 \mu \nu}\right)_{a b}\left(\hat{O}^{2 \sigma \lambda}\right)_{b a} g_{\mu \nu} g_{\sigma \lambda} J_{p+k}^{a b}\left(p+k, \tilde{m}_{f_{a}}, \tilde{m}_{f_{b}}\right)\right.
\end{aligned}
$$




$$
\begin{aligned}
& -\sum_{a, b, c}\left(\hat{O}^{1 \mu}\right)_{a b}\left(\hat{O}^{1 \nu}\right)_{b c}\left(\hat{O}^{2 \sigma \lambda}\right)_{c a} g_{\sigma \lambda} J_{\mu \nu}^{a b c}\left(p, k, \tilde{m}_{f_{a}}, \tilde{m}_{f_{b}}, \tilde{m}_{f_{c}}\right) \\
& \left.+\frac{1}{4} \sum_{a, b, c, d}\left(\hat{O}^{1 \mu}\right)_{a b}\left(\hat{O}^{1 \nu}\right)_{b c}\left(\hat{O}^{1 \sigma}\right)_{c d}\left(\hat{O}^{1 \lambda}\right)_{d a} J_{\mu \nu \sigma \lambda}^{a b c d}\left(p, k, r, \tilde{m}_{f_{a}}, \tilde{m}_{f_{b}}, \tilde{m}_{f_{c}}\right)\right)
\end{aligned}
$$

where the indexes $a, b, c$ and $d$ run from one to four corresponding to the four entries of the sfermion matrix in eq.(6) and the integrals and operators appearing in this formula are the ones given in Appendix A and B respectively.

From this formula we have obtained the sfermion contributions to the 4-point functions, $\Gamma_{\mu \nu \sigma \lambda}^{V_{1} V_{2} V_{3} V_{4}}$. In the case of the squarks we have presented the exact results for the $\Delta \Gamma_{\mu \nu \sigma \lambda}^{A A W^{+} W_{\tilde{q}}^{-}}$, $\Delta \Gamma_{\mu \nu \sigma \lambda}^{Z Z W_{\tilde{q}}^{+} W^{-}}, \Delta \Gamma_{\mu \nu \sigma \lambda}^{A Z W_{\tilde{q}}^{+} W^{-}}$and $\Delta \Gamma_{\mu \nu \sigma \lambda}^{W^{+} W^{-} W^{+} W^{-}}$in Appendix C. We have checked explicitely in addition that the other 4-point functions not shown in this appendix are finite as it corresponds to the Green's functions that do not have tree level contributions.

In order to get the asymptotic expressions for the effective action given in (58), one proceeds as in the previous sections. Notice that for the sfermions contributions to the four point part of the effective action it is not possible to write, directly, an expression equivalent to the one given in (45). In the first step after substituting just the asymptotic results of the integrals in eq.(58), one does not obtain yet a result proportional to the tree level vertex for the effective action and, one could think that it may be some non-decoupling effect in the Appelquist-Carazzone sense. However, this is not the case, and in order to conclude anything about the decoupling of sfermions in the 4-point functions one needs to go a step further and to compute the different contributions to the four-point Green's functions, which involve at the same time to perform the sums in the corresponding matrix indices and over the various types of sfermions. Finally, after performing these sums one gets the results for the sfermions contributions to the four-point functions that indeed show decoupling since they turn out to be proportional to the corresponding tree level contribution.

Analogously to the previous section, we write our results as:

$$
\Gamma_{\mu \nu \sigma \lambda}^{V_{1} V_{2} V_{3} V_{4}}=\Gamma_{0 \mu \nu \sigma \lambda}^{V_{1} V_{2} V_{3} V_{4}}+\Delta \Gamma_{\mu \nu \sigma \lambda}^{V_{1} V_{2} V_{3} V_{4}}
$$

where the outgoing momenta assignements are $V_{1}^{\mu}(p), V_{2}^{\nu}(k), V_{3}^{\sigma}(r)$ and $V_{4}^{\lambda}(t)$ and the different contributions to the effective action at tree level are defined by,

$$
\begin{gathered}
\Gamma_{0 \mu \nu \sigma \lambda}^{A A W^{+} W^{-}}=-e^{2} \beta_{\mu \nu \sigma \lambda} \quad, \quad \Gamma_{0 \mu \nu \sigma \lambda}^{A Z W^{+} W^{-}}=-g^{2} s_{W} c_{W} \beta_{\mu \nu \sigma \lambda}, \\
\Gamma_{0 \mu \nu \sigma \lambda}^{Z Z W^{+} W^{-}}=-g^{2} c_{W}^{2} \beta_{\mu \nu \sigma \lambda} \quad, \quad \Gamma_{0 \mu \nu \sigma \lambda}^{W^{+} W^{-} W^{+} W^{-}}=g^{2} \beta_{\mu \sigma \nu \lambda},
\end{gathered}
$$

with,

$$
\beta_{\mu \nu \sigma \lambda} \equiv\left[2 g_{\mu \nu} g_{\sigma \lambda}-g_{\mu \sigma} g_{\nu \lambda}-g_{\mu \lambda} g_{\nu \sigma}\right]
$$

The results for the squark contributions to the four-point functions that are different from 
zero are the following,

$$
\begin{aligned}
& \Delta \Gamma_{\mu \nu \sigma \lambda}^{A A W^{+} W^{-}}=-\frac{N_{c}}{6} \frac{e^{2} g^{2}}{16 \pi^{2}} \sum_{\tilde{q}}\left\{\beta_{\mu \nu \sigma \lambda} \Delta_{\epsilon}+g_{\mu \nu} g_{\sigma \lambda} g_{1}\left(\tilde{m}_{t_{1}}^{2}, \tilde{m}_{t_{2}}^{2}, \tilde{m}_{b_{1}}^{2}, \tilde{m}_{b_{2}}^{2}\right)\right. \\
& \left.+\left(g_{\mu \sigma} g_{\nu \lambda}+g_{\mu \lambda} g_{\nu \sigma}\right) g_{2}\left(\tilde{m}_{t_{1}}^{2}, \tilde{m}_{t_{2}}^{2}, \tilde{m}_{b_{1}}^{2}, \tilde{m}_{b_{2}}^{2}\right)\right\} \\
& =-\frac{N_{c}}{6} \frac{e^{2} g^{2}}{16 \pi^{2}} \beta_{\mu \nu \sigma \lambda} \sum_{\tilde{q}}\left\{\Delta_{\epsilon}-\log \frac{\hat{M}^{2}}{\mu_{o}^{2}}\right\}+G_{1 \mu \nu \sigma \lambda}\left[O\left(\frac{p^{2}}{\Sigma \tilde{m}^{2}}, \frac{\triangle \tilde{m}^{2}}{\Sigma \tilde{m}^{2}}\right)\right] \text {, } \\
& \Delta \Gamma_{\mu \nu \sigma \lambda}^{A Z W_{\tilde{q}}^{+} W^{-}}=-\frac{N_{c}}{6} \frac{e g^{3}}{16 \pi^{2}} \sum_{\tilde{q}}\left\{c_{W} \beta_{\mu \nu \sigma \lambda} \Delta_{\epsilon}+\frac{1}{c_{W}} g_{\mu \nu} g_{\sigma \lambda} g_{3}\left(\tilde{m}_{t_{1}}^{2}, \tilde{m}_{t_{2}}^{2}, \tilde{m}_{b_{1}}^{2}, \tilde{m}_{b_{2}}^{2}\right)\right. \\
& \left.+\frac{1}{c_{W}}\left(g_{\mu \sigma} g_{\nu \lambda}+g_{\mu \lambda} g_{\nu \sigma}\right) g_{4}\left(\tilde{m}_{t_{1}}^{2}, \tilde{m}_{t_{2}}^{2}, \tilde{m}_{b_{1}}^{2}, \tilde{m}_{b_{2}}^{2}\right)\right\} \\
& =-\frac{N_{c}}{6} \frac{e g^{3}}{16 \pi^{2}} c_{W} \beta_{\mu \nu \sigma \lambda} \sum_{\tilde{q}}\left\{\Delta_{\epsilon}-\log \frac{\hat{M}^{2}}{\mu_{o}^{2}}\right\}+G_{2 \mu \nu \sigma \lambda}\left[O\left(\frac{p^{2}}{\Sigma \tilde{m}^{2}}, \frac{\triangle \tilde{m}^{2}}{\Sigma \tilde{m}^{2}}\right)\right], \\
& \Delta \Gamma_{\mu \nu \sigma \lambda}^{Z Z W_{\tilde{q}}^{+} W^{-}}=-\frac{N_{c}}{6} \frac{g^{4}}{16 \pi^{2}} \sum_{\tilde{q}}\left\{c_{W}^{2} \beta_{\mu \nu \sigma \lambda} \Delta_{\epsilon}+\frac{1}{c_{W}^{2}} g_{\mu \nu} g_{\sigma \lambda} g_{5}\left(\tilde{m}_{t_{1}}^{2}, \tilde{m}_{t_{2}}^{2}, \tilde{m}_{b_{1}}^{2}, \tilde{m}_{b_{2}}^{2}\right)\right. \\
& \left.+\frac{1}{c_{W}^{2}}\left(g_{\mu \sigma} g_{\nu \lambda}+g_{\mu \lambda} g_{\nu \sigma}\right) g_{6}\left(\tilde{m}_{t_{1}}^{2}, \tilde{m}_{t_{2}}^{2}, \tilde{m}_{b_{1}}^{2}, \tilde{m}_{b_{2}}^{2}\right)\right\} \\
& =-\frac{N_{c}}{6} \frac{g^{4}}{16 \pi^{2}} c_{W}^{2} \beta_{\mu \nu \sigma \lambda} \sum_{\tilde{q}}\left\{\Delta_{\epsilon}-\log \frac{\hat{M}^{2}}{\mu_{o}^{2}}\right\}+G_{3 \mu \nu \sigma \lambda}\left[O\left(\frac{p^{2}}{\Sigma \tilde{m}^{2}}, \frac{\triangle \tilde{m}^{2}}{\Sigma \tilde{m}^{2}}\right)\right], \\
& \Delta \Gamma_{\mu \nu \sigma \lambda}^{W^{+} W^{-} W^{+} W_{\tilde{q}}^{-}}=-\frac{N_{c}}{3} \frac{g^{4}}{16 \pi^{2}} \sum_{\tilde{q}}\left\{\beta_{\mu \sigma \nu \lambda} \Delta_{\epsilon}+g_{\mu \nu} g_{\sigma \lambda} g_{7}\left(\tilde{m}_{t_{1}}^{2}, \tilde{m}_{t_{2}}^{2}, \tilde{m}_{b_{1}}^{2}, \tilde{m}_{b_{2}}^{2}\right)\right. \\
& \left.+\left(g_{\mu \sigma} g_{\nu \lambda}+g_{\mu \lambda} g_{\nu \sigma}\right) g_{8}\left(\tilde{m}_{t_{1}}^{2}, \tilde{m}_{t_{2}}^{2}, \tilde{m}_{b_{1}}^{2}, \tilde{m}_{b_{2}}^{2}\right)\right\} \\
& =-\frac{N_{c}}{3} \frac{g^{4}}{16 \pi^{2}} \beta_{\mu \sigma \nu \lambda} \sum_{\tilde{q}}\left\{\Delta_{\epsilon}-\log \frac{\hat{M}^{2}}{\mu_{o}^{2}}\right\}+G_{4 \mu \nu \sigma \lambda}\left[O\left(\frac{p^{2}}{\Sigma \tilde{m}^{2}}, \frac{\triangle \tilde{m}^{2}}{\Sigma \tilde{m}^{2}}\right)\right] \text {. }
\end{aligned}
$$

The functions $G_{k \mu \nu \sigma \lambda}$ and $g_{k}\left(\tilde{m}_{t_{1}}^{2}, \tilde{m}_{t_{2}}^{2}, \tilde{m}_{b_{1}}^{2}, \tilde{m}_{b_{2}}^{2}\right)(k=1, \ldots 4)$ are both finite, but the first ones vanish in our asymptotic limit whereas the second ones are different from zero in this limit. Therefore the latter contain all the potentially non-decoupling effects of the four-point functions. The explicit formulae of the $g_{k}$ functions $(k=1, \ldots 8)$ are collected in Appendix B. As a check of the previous functional computation we have also calculated all these four point functions by diagramatic methods and we have got the same results.

Notice that, if one takes the sum of the corresponding SUSY squared masses involved as the large parameter in the asymptotic expansion, $\Sigma \tilde{m}^{2}$, one gets that the dominant contributions to 
these $g_{k}$ functions are logarithmic. Generically, we can write,

$$
g_{k}\left(\tilde{m}_{t_{1}}^{2}, \tilde{m}_{t_{2}}^{2}, \tilde{m}_{b_{1}}^{2}, \tilde{m}_{b_{2}}^{2}\right)=O\left(\log \frac{\Sigma \tilde{m}^{2}}{\mu_{o}^{2}}\right)+O\left(\frac{\triangle \tilde{m}^{2}}{\Sigma \tilde{m}^{2}}\right),
$$

where $\triangle \tilde{m}^{2}$ denotes the various squared mass differences and, as in the previous cases, all the contributions of the type $O\left(\frac{\Delta \tilde{m}^{2}}{\Sigma \tilde{m}^{2}}\right)$ vanish in our asymptotic limit. Notice also that in the previous expressions of the four point functions that are given in terms of these $g_{k}$ functions, the decoupling is not manifest yet since the Lorentz tensorial structure is apparently not proportional to the tree level one. However, after rewritting these results in terms of the proper variable which in this case is given by,

$$
\hat{M}^{2} \equiv \frac{1}{4}\left(\tilde{m}_{t_{1}}^{2}+\tilde{m}_{t_{2}}^{2}+\tilde{m}_{b_{1}}^{2}+\tilde{m}_{b_{2}}^{2}\right)
$$

one finds out that the one loop corrections to the four point functions, $\Delta \Gamma$, in the asymptotic limit of large $\hat{M}^{2}$ are indeed proportional to the tree level contribution. This can be seen in the last lines of eqs. (62) to (65) respectively. Therefore the potentially non-decoupling effects in the four-point functions can also be absorbed into redefinitions of the coupling constants and wave functions.

Similar expressions are obtained for the sleptons sector doing the corresponding replacements mentioned at the end of subsection 4.1.

In summary, the results in this subsection show explicitely the decoupling of squarks and sleptons in the four-point functions.

\subsection{Inos contributions}

Here we consider the effective action for 4-point functions generated from the integration of charginos and neutralinos, which results after computing the corresponding last functional traces given in eq. (23). By inserting the operators and propagators of eqs. (12), (21) and (22) into equation (23) and after a lengthy calculation, that we do not present here for brevity, the inos contributions to the 4-point part of the effective action can be summarized as follows,

$$
\begin{aligned}
& \Gamma_{e f f}^{\tilde{\chi}}[V]_{[4]}=i \int \mathrm{d} \tilde{p} \mathrm{~d} \tilde{k} \mathrm{~d} \tilde{r} \mathrm{~d} \tilde{t}(2 \pi)^{4} \delta(p+k+r+t) \times \\
& \int d \widehat{q}\left[\frac { 1 } { 4 } \sum _ { i , j , k , l = 1 } ^ { 2 } \mathcal { G } ^ { i j k l } ( \tilde { M } _ { i } ^ { + } , \tilde { M } _ { j } ^ { + } , \tilde { M } _ { k } ^ { + } , \tilde { M } _ { l } ^ { + } ) \left\{q_{1}^{\alpha} q_{2}^{\beta} q_{3}^{\gamma} q_{4}^{\rho}(G \cdot O)_{1234}^{++++}\right.\right. \\
& +q_{1}^{\alpha} q_{2}^{\beta} \tilde{M}_{k}^{+} \tilde{M}_{l}^{+}(G \cdot O)_{12}^{++++}+q_{1}^{\alpha} q_{3}^{\gamma} \tilde{M}_{j}^{+} \tilde{M}_{l}^{+}(G \cdot O)_{13}^{++++}+q_{1}^{\alpha} q_{4}^{\rho} \tilde{M}_{j}^{+} \tilde{M}_{k}^{+}(G \cdot O)_{14}^{++++} \\
& +q_{2}^{\beta} q_{3}^{\gamma} \tilde{M}_{l}^{+} \tilde{M}_{i}^{+}(G \cdot O)_{23}^{++++}+q_{2}^{\beta} q_{4}^{\rho} \tilde{M}_{k}^{+} \tilde{M}_{i}^{+}(G \cdot O)_{24}^{++++}+q_{3}^{\gamma} q_{4}^{\rho} \tilde{M}_{i}^{+} \tilde{M}_{j}^{+}(G \cdot O)_{34}^{+++} \\
& \left.+\tilde{M}_{i}^{+} \tilde{M}_{j}^{+} \tilde{M}_{k}^{+} \tilde{M}_{l}^{+}(G \cdot O)^{++++}\right\} \\
& +\sum_{i=1}^{4} \sum_{j, k, l=1}^{2} \mathcal{G}^{i j k l}\left(\tilde{M}_{i}^{o}, \tilde{M}_{j}^{+}, \tilde{M}_{k}^{+}, \tilde{M}_{l}^{+}\right)\left\{q_{1}^{\alpha} q_{2}^{\beta} q_{3}^{\gamma} q_{4}^{\rho}(G \cdot O)_{1234}^{o+++}\right.
\end{aligned}
$$




$$
\begin{aligned}
& +q_{1}^{\alpha} q_{2}^{\beta} \tilde{M}_{k}^{+} \tilde{M}_{l}^{+}(G \cdot O)_{12}^{o+++}+q_{1}^{\alpha} q_{3}^{\gamma} \tilde{M}_{j}^{+} \tilde{M}_{l}^{+}(G \cdot O)_{13}^{o+++}+q_{1}^{\alpha} q_{4}^{\rho} \tilde{M}_{j}^{+} \tilde{M}_{k}^{+}(G \cdot O)_{14}^{o+++} \\
& +q_{2}^{\beta} q_{3}^{\gamma} \tilde{M}_{i}^{o} \tilde{M}_{l}^{+}(G \cdot O)_{23}^{o+++}+q_{2}^{\beta} q_{4}^{\rho} \tilde{M}_{i}^{o} \tilde{M}_{k}^{+}(G \cdot O)_{24}^{o+++}+q_{3}^{\gamma} q_{4}^{\rho} \tilde{M}_{i}^{o} \tilde{M}_{j}^{+}(G \cdot O)_{34}^{o+++} \\
& \left.+\tilde{M}_{i}^{o} \tilde{M}_{j}^{+} \tilde{M}_{k}^{+} \tilde{M}_{l}^{+}(G \cdot O)^{o+++}\right\} \\
& +\sum_{i, j=1}^{4} \sum_{k, l=1}^{2} \mathcal{G}^{i j k l}\left(\tilde{M}_{i}^{o}, \tilde{M}_{j}^{o}, \tilde{M}_{k}^{+}, \tilde{M}_{l}^{+}\right)\left\{q_{1}^{\alpha} q_{2}^{\beta} q_{3}^{\gamma} q_{4}^{\rho}(G \cdot O)_{1234}^{o o+++}\right. \\
& +q_{1}^{\alpha} q_{2}^{\beta} \tilde{M}_{k}^{+} \tilde{M}_{l}^{+}(G \cdot O)_{12}^{o o++}+q_{1}^{\alpha} q_{3}^{\gamma} \tilde{M}_{j}^{o} \tilde{M}_{l}^{+}(G \cdot O)_{13}^{o o++}+q_{1}^{\alpha} q_{4}^{\rho} \tilde{M}_{j}^{o} \tilde{M}_{k}^{+}(G \cdot O)_{14}^{o o++} \\
& +q_{2}^{\beta} q_{3}^{\gamma} \tilde{M}_{i}^{o} \tilde{M}_{l}^{+}(G \cdot O)_{23}^{o o++}+q_{2}^{\beta} q_{4}^{\rho} \tilde{M}_{i}^{o} \tilde{M}_{k}^{+}(G \cdot O)_{24}^{o o++}+q_{3}^{\gamma} q_{4}^{\rho} \tilde{M}_{i}^{o} \tilde{M}_{j}^{o}(G \cdot O)_{34}^{o o++} \\
& \left.+\tilde{M}_{i}^{o} \tilde{M}_{j}^{o} \tilde{M}_{k}^{+} \tilde{M}_{l}^{+}(G \cdot O)^{o o++}\right\} \\
& +\sum_{i, j, k=1}^{4} \sum_{l=1}^{2} \mathcal{G}^{i j k l}\left(\tilde{M} \tilde{M}_{i}^{o}, \tilde{M}_{j}^{o}, \tilde{M}_{k}^{o}, \tilde{M}_{l}^{+}\right)\left\{q_{1}^{\alpha} q_{2}^{\beta} q_{3}^{\gamma} q_{4}^{\rho}(G \cdot O)_{1234}^{o o o+}\right. \\
& +q_{1}^{\alpha} q_{2}^{\beta} \tilde{M}_{k}^{+} \tilde{M}_{l}^{+}(G \cdot O)_{12}^{o o o+}+q_{1}^{\alpha} q_{3}^{\gamma} \tilde{M}_{j}^{o} \tilde{M}_{l}^{+}(G \cdot O)_{13}^{o o o+}+q_{1}^{\alpha} q_{4}^{\rho} \tilde{M}_{j}^{o} \tilde{M}_{k}^{o}(G \cdot O)_{14}^{o o o+} \\
& +q_{2}^{\beta} q_{3}^{\gamma} \tilde{M}_{i}^{o} \tilde{M}_{l}^{+}(G \cdot O)_{23}^{o o o+}+q_{2}^{\beta} q_{4}^{\rho} \tilde{M}_{i}^{o} \tilde{M}_{k}^{o}(G \cdot O)_{24}^{o o o+}+q_{3}^{\gamma} q_{4}^{\rho} \tilde{M}_{i}^{o} \tilde{M}_{j}^{o}(G \cdot O)_{34}^{o o o+} \\
& \left.+\tilde{M}_{i}^{o} \tilde{M}_{j}^{o} \tilde{M}_{k}^{o} \tilde{M}_{l}^{+}(G \cdot O)^{o o o+}\right\} \\
& +\frac{1}{8} \sum_{i, j, k, l=1}^{4} \mathcal{G}^{i j k l}\left(\tilde{M}_{i}^{o}, \tilde{M}_{j}^{o}, \tilde{M}_{k}^{o}, \tilde{M}_{l}^{o}\right)\left\{q_{1}^{\alpha} q_{2}^{\beta} q_{3}^{\gamma} q_{4}^{\rho}(G \cdot O)_{1234}^{o o o o}\right. \\
& +q_{1}^{\alpha} q_{2}^{\beta} \tilde{M}_{k}^{o} \tilde{M}_{l}^{o}(G \cdot O)_{12}^{o o o o}+q_{1}^{\alpha} q_{3}^{\gamma} \tilde{M}_{j}^{o} \tilde{M}_{l}^{o}(G \cdot O)_{13}^{o o o o}+q_{1}^{\alpha} q_{4}^{\rho} \tilde{M}_{j}^{o} \tilde{M}_{k}^{o}(G \cdot O)_{14}^{o o o o} \\
& +q_{2}^{\beta} q_{3}^{\gamma} \tilde{M}_{i}^{o} \tilde{M}_{l}^{o}(G \cdot O)_{23}^{o o o o}+q_{2}^{\beta} q_{4}^{\rho} \tilde{M}_{i}^{o} \tilde{M}_{k}^{o}(G \cdot O)_{24}^{o o o o}+q_{3}^{\gamma} q_{4}^{\rho} \tilde{M}_{i}^{o} \tilde{M}_{j}^{o}(G \cdot O)_{34}^{o o o o} \\
& \left.+\tilde{M}_{i}^{o} \tilde{M}_{j}^{o} \tilde{M}_{k}^{o} \tilde{M}_{l}^{o}(G \cdot O)^{o o o o}\right\} \\
& +
\end{aligned}
$$

Analogously to eq. (52) we have used here the shorthand notation $(G \cdot O)$ for the various products of traces and operators whose explicit expressions are collected in Appendix B. Notice that there are some terms without subscripts which means there is no momentum contracted with the results of the traces. For example, in $(G \cdot O)^{++++}$, the superscripts denote the four charginos in the loop and the absence of subscripts indicates there is no contraction with any momenta. The definitions of the $q_{1}, q_{2}, q_{3}$ and $q_{4}$ momenta, as well as the generic function $\mathcal{G}^{i j k l}\left(\tilde{M}_{i}, \tilde{M}_{j}, \tilde{M}_{k}, \tilde{M}_{l}\right)$ are given by,

$$
q_{1} \equiv q, q_{2} \equiv q+p, q_{3} \equiv q+p+k, q_{4} \equiv q+p+k+r
$$

and

$$
\mathcal{G}^{i j k l}\left(\tilde{M}_{i}, \tilde{M}_{j}, \tilde{M}_{k}, \tilde{M}_{l}\right)=\frac{1}{\left[q_{1}^{2}-\tilde{M}_{i}^{2}\right]\left[q_{2}^{2}-\tilde{M}_{j}^{2}\right]\left[q_{3}^{2}-\tilde{M}_{k}^{2}\right]\left[q_{4}^{2}-\tilde{M}_{l}^{2}\right]}
$$

In order to obtain the exact contributions to one loop level from the inos sector, one must work out the corresponding Dirac traces in (67) and then write down the results in terms of the standard one-loop Feynman integrals. We have performed such computation but due to the length of the final expressions we prefer not to present these exact results here and to restrict ourselves to the presentation and discussion of just the corresponding asymptotic results. 
By starting with the exact result given in formula (67) and by inserting the asymptotic results of the corresponding integrals and coupling matrices we have derived the 4-point Green's functions from inos sector in the large masses limit. The results of these integrals have been given in Appendix A. After a lengthy calculation we can summarize the result for the four point part of the effective action in the asymptotic limit by the following expression:

$$
\begin{aligned}
& \Gamma_{e f f}^{\tilde{\chi}}[V]_{[4]}=\frac{4}{3} \pi^{2} \int \mathrm{d} \tilde{p} \mathrm{~d} \tilde{k} \mathrm{~d} \tilde{r} \mathrm{~d} \tilde{t} \delta(p+k+r+t) \times \\
& \sum_{i, j, k, l}\left\{\frac{1}{4}\left(\check{O}^{1}+\check{O}^{2}+\check{O}^{4}+\check{O}^{6}+\check{O}^{8}+\check{O}^{10}+\check{O}^{12}+\check{O}^{14}+\check{O}^{18}+\check{O}^{22}+\check{O}^{26}+\check{O}^{30}\right)_{i j k l}^{\mu \nu \sigma \lambda}\right. \\
& \left.+\frac{1}{8} \check{O}_{i j k l}^{38 \mu \nu \sigma \lambda}+\left(\check{O}^{46}+\check{O}^{50}+\check{O}^{54}+\check{O}^{58}+\check{O}^{60}+\check{O}^{68}+\check{O}^{76}+\check{O}^{84}\right)_{i j k l}^{\mu \nu \sigma \lambda}\right\} \times \\
& \left(\Delta_{\epsilon}-\log \frac{\tilde{M}_{i}^{2}+\tilde{M}_{j}^{2}+\tilde{M}_{k}^{2}+\tilde{M}_{l}^{2}}{4 \mu_{o}^{2}}\right) B_{\sigma \mu \nu \lambda},
\end{aligned}
$$

where $\beta_{\sigma \mu \nu \lambda}$ is the tree level tensor defined in eq. (61) but with the Lorentz indices being interchanged, and the operators $\check{O}_{i j k l}^{\mu \nu \sigma \lambda}$ are given in Appendix B.

At this point, we can already see that the asymptotic result from the inos sector is proportional to the tree level tensor after the proper symmetrization over the identical external fields and therefore, we can conclude that the inos decouple in the 4-point functions.

For completeness we present in the following the corresponding asymptotic results for the 4-point functions with specific external gauge bosons. After a lengthy computation we get,

$$
\begin{aligned}
\Delta \Gamma_{\mu \nu \sigma \lambda}^{A A W^{+} W_{\tilde{\chi}}^{-}} & =\frac{e^{2} g^{2}}{12 \pi^{2}} \beta_{\mu \nu \sigma \lambda}\left\{-\frac{3}{2} \Delta_{\epsilon}+g_{9}\left(\tilde{M}_{1}^{+}, \tilde{M}_{2}^{+}, \tilde{M}_{1}^{0}, \tilde{M}_{2}^{0}, \tilde{M}_{3}^{0}, \tilde{M}_{4}^{0}\right)\right\} \\
& +G_{5 \mu \nu \sigma \lambda}\left[O\left(\frac{p^{2}}{\Sigma \tilde{m}^{2}}, \frac{\triangle \tilde{m}^{2}}{\Sigma \tilde{m}^{2}}\right)\right], \\
\Delta \Gamma_{\mu \nu \sigma \lambda}^{A Z W_{\tilde{\chi}}^{+} W_{\tilde{\chi}}^{-}} & =\frac{e g^{3}}{12 \pi^{2}} \frac{1}{c_{W}} \beta_{\mu \nu \sigma \lambda}\left\{-\frac{3}{2} c_{W}^{2} \Delta_{\epsilon}+g_{10}\left(\tilde{M}_{1}^{+}, \tilde{M}_{2}^{+}, \tilde{M}_{1}^{0}, \tilde{M}_{2}^{0}, \tilde{M}_{3}^{0}, \tilde{M}_{4}^{0}\right)\right\} \\
& +G_{6 \mu \nu \sigma \lambda}\left[O\left(\frac{p^{2}}{\Sigma \tilde{m}^{2}}, \frac{\Delta \tilde{m}^{2}}{\Sigma \tilde{m}^{2}}\right)\right], \\
\Delta \Gamma_{\mu \nu \sigma \lambda}^{Z Z W^{+} W_{\tilde{\chi}}^{-}} & =-\frac{g^{4}}{48 \pi^{2}} \frac{1}{c_{W}^{2}} \beta_{\mu \nu \sigma \lambda}\left\{6 c_{W}^{4} \Delta_{\epsilon}+g_{11}\left(\tilde{M}_{1}^{+}, \tilde{M}_{2}^{+}, \tilde{M}_{1}^{0}, \tilde{M}_{2}^{0}, \tilde{M}_{3}^{0}, \tilde{M}_{4}^{0}\right)\right\} \\
& +G_{7 \mu \nu \sigma \lambda}\left[O\left(\frac{p^{2}}{\Sigma \tilde{m}^{2}}, \frac{\triangle \tilde{m}^{2}}{\Sigma \tilde{m}^{2}}\right)\right], \\
\Delta \Gamma_{\mu \nu \sigma \lambda}^{W^{+} W^{-} W^{+} W^{-}} \tilde{\chi}^{-} & =\frac{g^{4}}{96 \pi^{2}} \beta_{\mu \sigma \nu \lambda}\left\{10 \Delta_{\epsilon}+g_{12}\left(\tilde{M}_{1}^{+}, \tilde{M}_{2}^{+}, \tilde{M}_{1}^{0}, \tilde{M}_{2}^{0}, \tilde{M}_{3}^{0}, \tilde{M}_{4}^{0}\right)\right\} \\
& +G_{8 \mu \nu \sigma \lambda}\left[O\left(\frac{p^{2}}{\Sigma \tilde{m}^{2}}, \frac{\triangle \tilde{m}^{2}}{\Sigma \tilde{m}^{2}}\right)\right],
\end{aligned}
$$


where the functions $G_{k \mu \nu \sigma \lambda}$ and $g_{k}\left(\tilde{m}_{t_{1}}^{2}, \tilde{m}_{t_{2}}^{2}, \tilde{m}_{b_{1}}^{2}, \tilde{m}_{b_{2}}^{2}\right)(k=5, \ldots 8)$ are both finite, but the first ones vanish in our asymptotic limit whereas the second ones are different from zero in this limit. The explicit form of the latter can be found in Appendix B. In principle, their contain all the potentially non-decoupling effects of these four-point functions. As a check of the previous functional computation we have also calculated all these four point functions by diagramatic methods and we have got the same results.

As in the previous n-point Green's functions the one-loop corrections in eqs. (70) to (73) are also proportional to the tree level vertex and at the end, we can conclude that those potentially non-decoupling effects in the four-point functions can be reabsorbed into redefinitions of the various SM parameters. Therefore, we can guarantee that the decoupling of the inos in the 4-point Green's functions take place as well.

In addition, we have checked that after the proper symmetrization over the indices and momenta of the identical external fields, the $\Delta \Gamma^{A A A A}, \Delta \Gamma^{A A A Z}, \Delta \Gamma^{A A Z Z}, \Delta \Gamma^{A Z Z Z}$ and $\triangle \Gamma^{Z Z Z Z}$ contributions are exactly zero in our limit as it was expected since there are no corresponding tree level vertices. This is a rather non trivial check of our computation.

As can be seen from all the results in the present article and those obtained and discussed in [16], we have proved explicitely that the decoupling of sfermions, charginos and neutralinos in the two, three and four point functions with external gauge bosons do indeed occur and this decoupling proceeds by assuming that all the sparticle masses are large as compared to the electroweak scale but close to each other.

Once we have shown the decoupling of SUSY particles in the two, three and four point functions we can ask about the decoupling in the n-point functions, with $n>4$. In this case two important observations are in order. First, due to the renormalizability of the MSSM there are no divergent contributions to the five or higher point functions since those functions vanish at the tree level and we are working in renormalizable gauges. Thus, those Green's functions are finite and so are the sum of the Feynman integrals corresponding to each given Green's function. In this case their asymptotic behaviour in the above defined region can trivially be obtained. Then it is inmediate to check that the decoupling of the SUSY particles also takes place.

\section{Conclusions}

In this work we have studied the decoupling properties of the SUSY particles appearing in the MSSM. In particular we have shown that the SM can be considered as the low energy effective theory of the MSSM in the limit where the sparticle masses are large. Our proof of decoupling in the Green's functions with external gauge bosons is quite general and does not depend on the particular form of the soft breaking terms since it is performed completly in terms of the SUSY masses. The decoupling is shown in the sense of the Appelquist-Carazzone Theorem. By this we mean that in the appropriate asymptotic region of large SUSY masses considered in this work, 
the effect of the SUSY particles on the gauge boson Green's functions can be absorbed in the SM parameters and gauge boson wave functions or else they correspond to new terms which are suppressed by negative powers of the SUSY masses.

However, in addition to the SUSY particles, the MSSM has also other particles which are not present in the SM. These are the extra Higgs scalar doublets that must be added to the MSSM in order to produce fermion masses and that through their SUSY partners give rise to an anomaly free theory. In order to provide a complete proof that the SM is really the low energy effective theory of the MSSM one should show that these extra scalars also decouple in the above mentioned sense. There are some indications that this could be the case. In addition, one must also study not just the Green's functions with external gauge bosons but also with all the possible SM particles in the external legs. Particularly interesting in the context of decoupling could be the Green's functions with external heavy fermions where due to the enhacement effect of the heavy fermion masses the decoupling of the SUSY particles could either not occur or to proceed much slowly. Work in progress in these directions is being done but the results will be presented elsewhere.

\section{Acknowledgements.}

This work has been partially supported by the Spanish Ministerio de Educación y Cultura under projects CICYT AEN97-1678 and AEN93-0776, and the fellowship AP95 00503301.

\section{Appendix A.}

In this appendix we give the definition of the one-loop integrals that have been used in the computation of the three and four-point functions and their results in the large masses limit. The one-loop integrals contributing to the two-point functions were presented in our previous work [16] to which we refer the reader for completeness. As all these integrals can be written in terms of the standard scalar and tensor integrals [17], we start by reviewing the definition of these standard 2-, 3- and 4-point integrals in the following. From now on, we denote:

$$
\int d \widehat{q} \equiv \int \frac{d^{D} q}{(2 \pi)^{D}} \mu_{o}^{4-D} .
$$

- Standard integrals.

$$
\begin{aligned}
A_{0}\left(m_{1}\right) & \equiv-i 16 \pi^{2} \int d \widehat{q} \frac{1}{D_{1}} \\
B_{0, \mu, \mu \nu}\left(p, m_{1}, m_{2}\right) & \equiv-i 16 \pi^{2} \int d \widehat{q} \frac{\left\{1, q_{\mu}, q_{\mu} q_{\nu}\right\}}{D_{1} D_{2}}
\end{aligned}
$$




$$
\begin{aligned}
C_{0, \mu, \mu \nu, \mu \nu \sigma}\left(p, k, m_{1}, m_{2}, m_{3}\right) & \equiv-i 16 \pi^{2} \int d \widehat{q} \frac{\left\{1, q_{\mu}, q_{\mu} q_{\nu}, q_{\mu} q_{\nu} q_{\sigma}\right\}}{D_{1} D_{2} D_{3}}, \\
D_{0, \mu, \mu \nu, \mu \nu \sigma, \mu \nu \sigma \lambda}\left(p, k, r, m_{1}, m_{2}, m_{3}, m_{4}\right) & \equiv-i 16 \pi^{2} \int d \widehat{q} \frac{\left\{1, q_{\mu}, q_{\mu} q_{\nu}, q_{\mu} q_{\nu} q_{\sigma}, q_{\mu} q_{\nu} q_{\sigma} q_{\lambda}\right\}}{D_{1} D_{2} D_{3} D_{4}},
\end{aligned}
$$

with the denominators given by,

$$
\begin{aligned}
& D_{1}=\left[q^{2}-m_{1}^{2}\right] \\
& D_{2}=\left[(q+p)^{2}-m_{2}^{2}\right] \\
& D_{3}=\left[(q+p+k)^{2}-m_{3}^{2}\right] \\
& D_{4}=\left[(q+p+k+r)^{2}-m_{4}^{2}\right]
\end{aligned}
$$

- One-loop integrals.

The 3-point integrals appearing in eqs. (44), (C.7) and (C.8) are given in terms of the standard integrals by,

$$
\begin{aligned}
& T_{\mu}^{a b}\left(p, \tilde{m}_{f_{a}}, \tilde{m}_{f_{b}}\right)=2 B_{\mu}\left(p, \tilde{m}_{f_{a}}, \tilde{m}_{f_{b}}\right)+p_{\mu} B_{0}\left(p, \tilde{m}_{f_{a}}, \tilde{m}_{f_{b}}\right), \\
& T_{\mu \nu \sigma}^{a b c}\left(p, k, \tilde{m}_{f_{a}}, \tilde{m}_{f_{b}}, \tilde{m}_{f_{c}}\right)=\left\{8 C_{\mu \nu \sigma}+4\left[(p+k)_{\sigma} C_{\mu \nu}+(2 p+k)_{\nu} C_{\mu \sigma}+p_{\mu} C_{\nu \sigma}\right]\right. \\
& +2\left[(p+k)_{\sigma}(2 p+k)_{\nu} C_{\mu}+p_{\mu}(p+k)_{\sigma} C_{\nu}+p_{\mu}(2 p+k)_{\nu} C_{\sigma}\right] \\
& \left.+p_{\mu}(2 p+k)_{\nu}(p+k)_{\sigma} C_{0}\right\}\left(p, k, \tilde{m}_{f_{a}}, \tilde{m}_{f_{b}}, \tilde{m}_{f_{c}}\right) \text {, } \\
& \mathcal{T}_{\mu \nu \sigma}^{i j k}\left(p, k, \tilde{m}_{i}, \tilde{m}_{j}, \tilde{m}_{k}\right)=\left\{4 C_{\mu \nu \sigma}+2(k+p)_{\sigma} C_{\mu \nu}+2(k+2 p)_{\nu} C_{\mu \sigma}+2 p_{\mu} C_{\nu \sigma}\right. \\
& +\left(k_{\nu} p_{\mu}+k_{\mu} p_{\nu}+2 p_{\mu} p_{\nu}\right) C_{\sigma}+\left(k_{\nu} p_{\sigma}+k_{\sigma} p_{\nu}+2 p_{\nu} p_{\sigma}\right) C_{\mu} \\
& +\left(k_{\sigma} p_{\nu}-k_{\mu} p_{\sigma}\right) C_{\nu} \\
& \text { - } g_{\alpha \beta}\left[g_{\mu \nu} C_{\alpha \beta \sigma}+g_{\sigma \nu} C_{\alpha \beta \mu}+g_{\sigma \mu} C_{\alpha \beta \nu}+2 g_{\sigma \nu} C_{\beta \mu}(k+p)_{\alpha}\right. \\
& +C_{\alpha \beta}\left(g_{\sigma \mu}(k+2 p)_{\nu}+g_{\mu \nu} k_{\sigma}-g_{\sigma \nu} k_{\mu}\right)+2 g_{\mu \nu} C_{\beta \sigma} p_{\alpha} \\
& +C_{\beta}\left(g_{\sigma \mu} p_{\alpha}(k+2 p)_{\nu}+g_{\sigma \mu} k_{\alpha} p_{\nu}\right. \\
& \left.+g_{\mu \nu}\left(k_{\sigma} p_{\alpha}-k_{\alpha} p_{\sigma}\right)+g_{\sigma \nu}\left(k_{\alpha} p_{\mu}-k_{\mu} p_{\alpha}\right)\right) \\
& \left.\left.+p_{\beta}(k+p)_{\alpha}\left(g_{\mu \nu} C_{\sigma}+g_{\sigma \nu} C_{\mu}-g_{\sigma \mu} C_{\nu}\right)\right]\right\}\left(p, k, \tilde{m}_{i}, \tilde{m}_{j}, \tilde{m}_{k}\right), \\
& \mathcal{I}_{\mu \nu \sigma}^{i j k}\left(p, k, \tilde{m}_{i}, \tilde{m}_{j}, \tilde{m}_{k}\right)=\left\{g_{\mu \nu} C_{\sigma}+g_{\sigma \nu} C_{\mu}-g_{\sigma \mu} C_{\nu}\right\}\left(p, k, \tilde{m}_{i}, \tilde{m}_{j}, \tilde{m}_{k}\right) \\
& \mathcal{P}_{\mu \nu \sigma}^{i j k}\left(p, k, \tilde{m}_{i}, \tilde{m}_{j}, \tilde{m}_{k}\right)=\left\{g_{\sigma \nu}\left(p_{\mu} C_{0}+C_{\mu}\right)+g_{\sigma \mu}\left(p_{\nu} C_{0}+C_{\nu}\right)\right. \\
& \left.-g_{\mu \nu}\left(p_{\sigma} C_{0}+C_{\sigma}\right)\right\}\left(p, k, \tilde{m}_{i}, \tilde{m}_{j}, \tilde{m}_{k}\right) \text {, } \\
& \mathcal{J}_{\mu \nu \sigma}^{i j k}\left(p, k, \tilde{m}_{i}, \tilde{m}_{j}, \tilde{m}_{k}\right)=\left\{g_{\mu \nu}\left(C_{\sigma}+(k+p)_{\sigma} C_{0}\right)-g_{\sigma \nu}\left(C_{\mu}+(k+p)_{\mu} C_{0}\right)\right. \\
& \left.+g_{\sigma \mu}\left(C_{\nu}+(k+p)_{\nu} C_{0}\right)\right\}\left(p, k, \tilde{m}_{i}, \tilde{m}_{j}, \tilde{m}_{k}\right) \text {, }
\end{aligned}
$$


where the variables within the last parentheses correspond to the arguments of the corresponding integrals.

Now, we present the 4-point integrals appearing in the computation of the four-point functions. Let us begin for those involved in the computation of sfermions contributions that is, in eqs. (58):

$$
\begin{aligned}
J_{p+k}^{a b}\left(p+k, \tilde{m}_{f_{a}}, \tilde{m}_{f_{b}}\right) & =B_{0}\left(p+k, \tilde{m}_{f_{a}}, \tilde{m}_{f_{b}}\right), \\
J_{\mu \nu}^{a b c}\left(p, k, \tilde{m}_{f_{a}}, \tilde{m}_{f_{b}}, \tilde{m}_{f_{c}}\right) & =\left\{4 C_{\mu \nu}+2(k+2 p)_{\nu} C_{\mu}\right. \\
& \left.+2 p_{\mu} C_{\nu}+p_{\mu}(k+2 p)_{\nu} C_{0}\right\}\left(p, k, \tilde{m}_{f_{a}}, \tilde{m}_{f_{b}}, \tilde{m}_{f_{c}}\right), \quad(\mathrm{A} .1 \\
J_{\mu \nu \sigma \lambda}^{a b c d}\left(p, k, r, \tilde{m}_{f_{a}}, \tilde{m}_{f_{b}}, \tilde{m}_{f_{c}}, \tilde{m}_{f_{d}}\right) & =\left\{16 D_{\mu \nu \sigma \lambda}+8(k+p+r)_{\lambda} D_{\mu \nu \sigma}+8 p_{\mu} D_{\nu \sigma \lambda}\right. \\
& +8(2 k+2 p+r)_{\sigma} D_{\mu \nu \lambda}+8(k+2 p)_{\nu} D_{\mu \sigma \lambda} \\
& +4(2 k+2 p+r)_{\sigma}(k+p+r)_{\lambda}\left(D_{\mu \nu}+p_{\mu} D_{\nu}\right) \\
& +2(2 k+2 p+r)_{\sigma}(k+2 p)_{\nu}\left(2 D_{\mu \lambda}+p_{\mu} D_{\lambda}\right) \\
& +2(k+2 p)_{\nu}(k+p+r)_{\lambda}\left(2 D_{\mu \sigma}+p_{\mu} D_{\sigma}\right) \\
& +2(2 k+2 p+r)_{\sigma}(k+2 p)_{\nu}(k+p+r)_{\lambda} D_{\mu} \\
& +p_{\mu}(k+2 p)_{\nu}(k+p+r)_{\lambda}(2 k+2 p+r)_{\sigma} D_{0} \\
& +4 p_{\mu}(k+p+r)_{\lambda} D_{\nu \sigma}+4 p_{\mu}(k+2 p)_{\nu} D_{\sigma \lambda} \\
& \left.+4 p_{\mu}(2 k+2 p+r)_{\sigma} D_{\nu \lambda}\right\}\left(p, k, \tilde{m}_{f_{a}}, \tilde{m}_{f_{b}}, \tilde{m}_{f_{c}}, \tilde{m}_{f_{d}}\right) .
\end{aligned}
$$

- Asymptotic results.

As we said before, we compute all the integrals in the large masses limit by using the mTheorem [15]. Some examples of the applicability of this theorem to the present context of decoupling of SUSY particles can be found in ref. [16].

We present in the following the results for the standard one-loop integrals in the limit of heavy SUSY particles. In taking this limit we require in addition that the differences of masses be always smaller than their sums, i.e $\tilde{m}^{2} \gg k^{2}$ and $\left|\tilde{m}_{i}^{2}-\tilde{m}_{j}^{2}\right| \ll\left|\tilde{m}_{i}^{2}+\tilde{m}_{j}^{2}\right|$. The results of the standard integrals in our asymptotic limit are as follows,

$$
\begin{aligned}
A_{0}\left(m_{1}\right) & =\left(\Delta_{\epsilon}+1-\log \frac{m_{1}^{2}}{\mu_{o}^{2}}\right) m_{1}^{2}, \\
B_{0}\left(p, m_{1}, m_{2}\right) & =\left(\Delta_{\epsilon}-\log \frac{m_{1}^{2}+m_{2}^{2}}{2 \mu_{o}^{2}}\right), \\
B_{\mu}\left(p, m_{1}, m_{2}\right) & =-\frac{1}{2} p_{\mu}\left(\Delta_{\epsilon}-\log \frac{m_{1}^{2}+m_{2}^{2}}{2 \mu_{o}^{2}}\right),
\end{aligned}
$$




$$
\begin{aligned}
& B_{\mu \nu}\left(p, m_{1}, m_{2}\right)=\frac{1}{4}\left(m_{1}^{2}+m_{2}^{2}\right)\left(\Delta_{\epsilon}+1-\log \frac{m_{1}^{2}+m_{2}^{2}}{2 \mu_{o}^{2}}\right) g_{\mu \nu} \\
& -\frac{1}{12} p^{2}\left(\Delta_{\epsilon}-\log \frac{m_{1}^{2}+m_{2}^{2}}{2 \mu_{o}^{2}}\right) g_{\mu \nu} \\
& +\frac{1}{3} p_{\mu} p_{\nu}\left(\Delta_{\epsilon}-\log \frac{m_{1}^{2}+m_{2}^{2}}{2 \mu_{o}^{2}}\right) \text {, } \\
& C_{0}\left(p, k, m_{1}, m_{2}, m_{3}\right)=0, C_{\mu}\left(p, k, m_{1}, m_{2}, m_{3}\right)=0, \\
& C_{\mu \nu}\left(p, k, m_{1}, m_{2}, m_{3}\right)=\frac{1}{4}\left(\Delta_{\epsilon}-\log \frac{m_{1}^{2}+m_{2}^{2}+m_{3}^{2}}{3 \mu_{o}^{2}}\right) g_{\mu \nu}, \\
& C_{\mu \nu \sigma}\left(p, k, m_{1}, m_{2}, m_{3}\right)=-\frac{1}{12}(2 p+k)_{\rho}\left(\Delta_{\epsilon}-\log \frac{m_{1}^{2}+m_{2}^{2}+m_{3}^{2}}{3 \mu_{o}^{2}}\right) \times \\
& {\left[g_{\mu \nu} g_{\sigma \rho}+g_{\mu \sigma} g_{\nu \rho}+g_{\mu \rho} g_{\nu \sigma}\right]} \\
& D_{0}\left(p, k, r, m_{1}, m_{2}, m_{3}, m_{4}\right)=0, D_{\mu}\left(p, k, r, m_{1}, m_{2}, m_{3}, m_{4}\right)=0, \\
& D_{\mu \nu}\left(p, k, r, m_{1}, m_{2}, m_{3}, m_{4}\right)=0, D_{\mu \nu \sigma}\left(p, k, r, m_{1}, m_{2}, m_{3}, m_{4}\right)=0 \text {, } \\
& D_{\mu \nu \sigma \lambda}\left(p, k, r, m_{1}, m_{2}, m_{3}, m_{4}\right)=\frac{1}{24}\left(\Delta_{\epsilon}-\log \frac{m_{1}^{2}+m_{2}^{2}+m_{3}^{2}+m_{4}^{2}}{4 \mu_{o}^{2}}\right) \times \\
& {\left[g_{\mu \nu} g_{\sigma \lambda}+g_{\mu \sigma} g_{\nu \lambda}+g_{\mu \lambda} g_{\nu \sigma}\right] \text {. }}
\end{aligned}
$$

The corrections to these formulae are suppressed by inverse powers of the sums of the corresponding squared masses and vanish in the asymptotic large masses limit.

Finally, notice that the results for the 3- and 4-point integrals appearing in our calculations can be easily obtained from the above formulae by substitution in eqs. (A.3) to (A.11) correspondingly. We will not present here these results for brevity.

This complete our analysis and results of the 3 and 4-point integrals that have appeared in the present work.

\section{Appendix B.}

In this appendix we collect the definitions of all the operators that have been introduced in this work as well as the different functions, $f_{i}(i=1 \ldots 4)$, and $g_{i}(i=1 \ldots 12)$, appearing in the asymptotic results for the 3 and 4-point Green's functions respectively. Since we work in 
the momentum space all these operators are functions of the corresponding momenta. Thus, for instance, the three-point-function operator given by $\hat{O}^{\mu \nu \sigma} \sim V_{1}^{\mu} V_{2}^{\nu} V_{3}^{\sigma}$ really means $\hat{O}^{\mu \nu \sigma} \sim$ $V_{1}^{\mu}(p) V_{2}^{\nu}(k) V_{3}^{\sigma}(r)$ and similarly for the other operators. In the following we omit this explicit momentum dependence for brevity.

The operators in eqs. (44) are defined by,

$$
\begin{aligned}
\hat{O}^{1 \mu} & =e A^{\mu} \hat{Q}_{f}+\frac{g}{c_{w}} Z^{\mu} \hat{G}_{f}+\frac{g}{\sqrt{2}} W^{+\mu} \Sigma_{f}^{t b}+\frac{g}{\sqrt{2}} W^{-\mu} \Sigma_{f}^{b t}, \\
\hat{O}^{2 \mu \nu} & =e^{2} \hat{Q}_{f}^{2} A^{\mu} A^{\nu}+\frac{2 g e}{c_{w}} A^{\mu} Z^{\nu} \hat{Q}_{f} \hat{G}_{f}+\frac{g^{2}}{c_{w}^{2}} \hat{G}_{f}^{2} Z^{\mu} Z^{\nu}+\frac{g^{2}}{2} \Sigma_{f} W^{\mu+} W^{\nu-} \\
& +\frac{e g}{\sqrt{2}} y_{\tilde{f}} A^{\mu}\left(W^{\nu+} \Sigma_{f}^{t b}+W^{\nu-} \Sigma_{f}^{b t}\right)-\frac{g^{2}}{\sqrt{2}} y_{\tilde{f}} \frac{s_{w}^{2}}{c_{w}} Z^{\mu}\left(W^{\nu+} \Sigma_{f}^{t b}+W^{\nu-} \Sigma_{f}^{b t}\right) .
\end{aligned}
$$

In order to write a general expression for the three and four-point functions from inos contributions that have been presented in eqs. (52) and (67), we have introduced the shorthand notation $(G \cdot O)$, which we give explicitely in the following. For this purpose we use the compact notation:

$$
\begin{aligned}
G_{\mu \nu \alpha \sigma} & \equiv \operatorname{Tr}\left[\gamma_{\mu} \gamma_{\nu} \gamma_{\alpha} \gamma_{\sigma}\right] \\
G_{\alpha \mu \beta \nu \gamma \sigma} & \equiv \operatorname{Tr}\left[\gamma_{\alpha} \gamma_{\mu} \gamma_{\beta} \gamma_{\nu} \gamma_{\gamma} \gamma_{\sigma}\right] \\
G_{\alpha \mu \beta \nu \gamma \sigma \rho \lambda} & \equiv \operatorname{Tr}\left[\gamma_{\alpha} \gamma_{\mu} \gamma_{\beta} \gamma_{\nu} \gamma_{\gamma} \gamma_{\sigma} \gamma_{\rho} \gamma_{\lambda}\right]
\end{aligned}
$$

The expressions for each $(G \cdot O)$ term in eq. (52) for the three-point functions are:

$$
\begin{aligned}
& (G \cdot O)_{123}^{+++}=G_{\alpha \mu \beta \nu \gamma \sigma}\left(\hat{O}^{1}+\hat{O}^{2}+\hat{O}^{4}+\hat{O}^{6}+\hat{O}^{8}\right) \\
& (G \cdot O)_{1}^{+++}=G_{\alpha \mu \nu \sigma}\left(\hat{O}^{1}+\hat{O}^{3}+\hat{O}^{5}+\hat{O}^{6}+\hat{O}^{9}\right), \\
& (G \cdot O)_{2}^{+++}=G_{\mu \alpha \nu \sigma}\left(\hat{O}^{1}+\hat{O}^{3}+\hat{O}^{4}+\hat{O}^{7}+\hat{O}^{10}\right), \\
& (G \cdot O)_{3}^{+++}=G_{\mu \nu \alpha \sigma}\left(\hat{O}^{1}+\hat{O}^{2}+\hat{O}^{5}+\hat{O}^{7}+\hat{O}^{11}\right), \\
& (G \cdot O)_{123}^{o++}=G_{\alpha \mu \beta \nu \gamma \sigma}\left(\hat{O}^{16}+\hat{O}^{18}\right),(G \cdot O)_{1}^{o++}=G_{\alpha \mu \nu \sigma}\left(\hat{O}^{16}+\hat{O}^{19}\right) \\
& (G \cdot O)_{2}^{o++}=G_{\mu \alpha \nu \sigma}\left(\hat{O}^{17}+\hat{O}^{20}\right),(G \cdot O)_{3}^{o++}=G_{\mu \nu \alpha \sigma}\left(\hat{O}^{17}+\hat{O}^{21}\right) \\
& (G \cdot O)_{123}^{o o+}=G_{\alpha \mu \beta \nu \gamma \sigma} \hat{O}^{22},(G \cdot O)_{1}^{o o+}=G_{\alpha \mu \nu \sigma} \hat{O}^{23}, \\
& (G \cdot O)_{2}^{o o+}=G_{\mu \alpha \nu \sigma} \hat{O}^{24},(G \cdot O)_{3}^{o o+}=G_{\mu \nu \alpha \sigma} \hat{O}^{25}, \\
& (G \cdot O)_{123}^{o o o}=G_{\alpha \mu \beta \nu \gamma \sigma} \hat{O}^{12},(G \cdot O)_{1}^{o o o}=G_{\alpha \mu \nu \sigma} \hat{O}^{13}, \\
& (G \cdot O)_{2}^{o o o}=G_{\mu \alpha \nu \sigma} \hat{O}^{14},(G \cdot O)_{3}^{o o o}=G_{\mu \nu \alpha \sigma} \hat{O}^{15},
\end{aligned}
$$

where the traces are given in $(\mathbb{B . 2})$ and the operators whose indices have been omitted here for shortness are given by,

$$
\hat{O}_{i j k}^{1 \mu \nu \sigma}=-e^{3} A_{\mu} A_{\nu} A_{\sigma} \delta_{i j} \delta_{j k} \delta_{k i}+e^{2} \frac{g}{2 c_{W}}\left[A_{\mu} A_{\nu} Z_{\sigma} \delta_{i j} \delta_{j k}\left(O^{\prime}{ }_{L}+O^{\prime}\right)_{k i}\right.
$$




$$
\begin{aligned}
& \left.+A_{\mu} Z_{\nu} A_{\sigma} \delta_{i j} \delta_{k i}\left(O_{L}^{\prime}+O_{R}^{\prime}\right)_{j k}+Z_{\mu} A_{\nu} A_{\sigma} \delta_{j k} \delta_{k i}\left(O_{L}^{\prime}+O_{R}^{\prime}\right)_{i j}\right] \\
& \hat{O}_{i j k}^{2(3) \mu \nu \sigma}=-e \frac{g^{2}}{2 c_{W}^{2}} A_{\mu} Z_{\nu} Z_{\sigma} \delta_{i j}\left(O_{L_{k i}}^{\prime} O_{L(R)_{j k}}^{\prime}+O_{R_{k i}}^{\prime} O_{R(L)_{j k}}^{\prime}\right) \text {, } \\
& \hat{O}_{i j k}^{4(5) \mu \nu \sigma}=-e \frac{g^{2}}{2 c_{W}^{2}} A_{\sigma} Z_{\mu} Z_{\nu} \delta_{k i}\left(O_{L_{i j}}^{\prime} O_{L(R)_{j k}}^{\prime}+O_{R_{i j}}^{\prime} O_{R(L)_{j k}}^{\prime}\right) \text {, } \\
& \hat{O}_{i j k}^{6(7) \mu \nu \sigma}=-e \frac{g^{2}}{2 c_{W}^{2}} A_{\nu} Z_{\mu} Z_{\sigma} \delta_{j k}\left(O_{L_{i j}}^{\prime} O_{L(R)_{k i}}^{\prime}+O_{R_{i j}}^{\prime} O_{R(L)_{k i}}^{\prime}\right) \text {, } \\
& \hat{O}_{i j k}^{8(9) \mu \nu \sigma}=\frac{g^{3}}{2 c_{W}{ }^{3}} Z_{\mu} Z_{\nu} Z_{\sigma}\left(O_{L_{i j}}^{\prime} O_{L(R)_{j k}}^{\prime} O_{L_{k i}}^{\prime}+O_{R_{i j}}^{\prime} O_{R(L)_{j k}}^{\prime} O_{R_{k i}}^{\prime}\right) \text {, }
\end{aligned}
$$

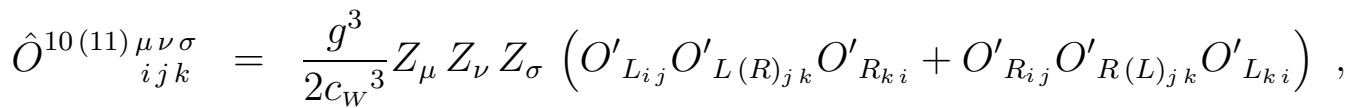

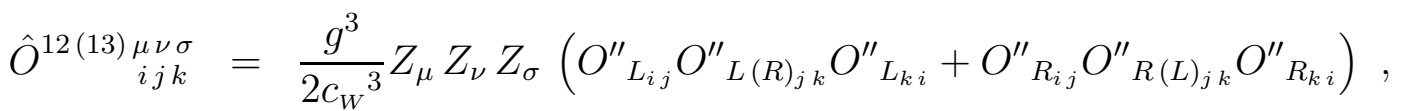

$$
\begin{aligned}
& \hat{O}^{14(15) \underset{i j k}{\mu \nu \sigma}}=\frac{g^{3}}{2 c_{W}{ }^{3}} Z_{\mu} Z_{\nu} Z_{\sigma}\left(O^{\prime \prime}{ }_{i j} O^{\prime \prime}{ }_{L(R)_{j}} O^{\prime \prime}{ }_{R_{k i}}+O^{\prime \prime}{ }_{R_{i j}} O^{\prime \prime}{ }_{R}(L)_{j k} O^{\prime \prime} L_{k i}\right) \\
& \hat{O}^{16(17) \mu \nu \sigma} \underset{i j k}{\mu \nu}=-e \frac{g^{2}}{2} A_{\nu} W_{\mu}^{-} W_{\sigma}^{+} \delta_{j k}\left(O_{L_{i j}} O_{L(R)_{k i}}^{+}+O_{R_{i j}} O_{R(L)_{k i}}^{+}\right), \\
& \hat{O}^{18(19) \underset{i j k}{\mu \nu \sigma}}=\frac{g^{3}}{2 c_{W}} Z_{\nu} W_{\mu}^{-} W_{\sigma}^{+}\left(O_{L_{i j}} O_{L(R)_{j k}}^{\prime} O_{L_{k i}}^{+}+O_{R_{i j}} O_{R(L)_{j k}}^{\prime} O_{R_{k i}}^{+}\right) \\
& \hat{O}^{20(21) \underset{i j k}{\mu \nu \sigma}}=\frac{g^{3}}{2 c_{W}} Z_{\nu} W_{\mu}^{-} W_{\sigma}^{+}\left(O_{L_{i j}} O_{L(R)_{j k}}^{\prime} O_{R_{k i}}^{+}+O_{R_{i j}} O_{R(L)_{j k}}^{\prime} O_{L_{k i}}^{+}\right) \\
& \hat{O}^{22(23) \underset{i j k}{\mu \nu \sigma}}=\frac{g^{3}}{2 c_{W}} Z_{\mu} W_{\nu}^{-} W_{\sigma}^{+}\left(O^{\prime \prime}{ }_{L_{i j}} O_{L(R)_{j k}} O_{L_{k i}}^{+}+O^{\prime \prime}{ }_{R_{i j}} O_{R(L)_{j k}} O_{R_{k i}}^{+}\right) \text {, }
\end{aligned}
$$

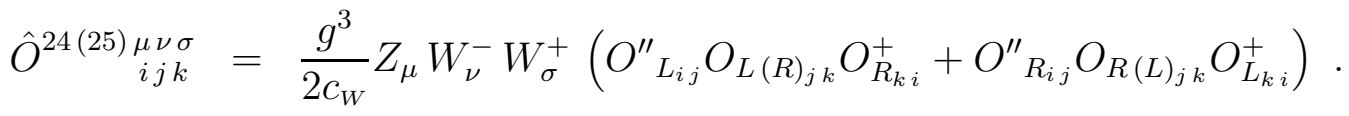

The generic terms $(G \cdot O)$ in the inos contributions to the four-point functions given in eq. (67) can be written as:

$$
\begin{aligned}
(G \cdot O)_{1234}^{++++} & =G_{\alpha \mu \beta \nu \gamma \sigma \rho \lambda}\left(\check{O}^{1}+\check{O}^{2}+\check{O}^{4}+\check{O}^{6}+\check{O}^{8}+\check{O}^{10}+\check{O}^{12}+\check{O}^{14}+\check{O}^{18}\right. \\
& \left.+\check{O}^{22}+\check{O}^{26}+\check{O}^{30}\right) \\
(G \cdot O)_{14}^{++++} & =G_{\alpha \mu \nu \sigma \rho \lambda}\left(\check{O}^{1}+\check{O}^{2}+\check{O}^{5}+\check{O}^{6}+\check{O}^{9}+\check{O}^{10}+\check{O}^{13}+\check{O}^{17}+\check{O}^{18}\right. \\
& \left.+\check{O}^{23}+\check{O}^{27}+\check{O}^{31}\right), \\
(G \cdot O)_{13}^{++++} & =G_{\alpha \mu \nu \gamma \sigma \lambda}\left(\check{O}^{1}+\check{O}^{3}+\check{O}^{5}+\check{O}^{6}+\check{O}^{8}+\check{O}^{11}+\check{O}^{13}+\check{O}^{16}+\check{O}^{19}\right. \\
& \left.+\check{O}^{23}+\check{O}^{29}+\check{O}^{34}\right), \\
(G \cdot O)_{12}^{++++} & =G_{\alpha \mu \beta \nu \sigma \lambda}\left(\check{O}^{1}+\check{O}^{3}+\check{O}^{4}+\check{O}^{6}+\check{O}^{9}+\check{O}^{11}+\check{O}^{12}+\check{O}^{15}+\check{O}^{19}\right. \\
& \left.+\check{O}^{22}+\check{O}^{28}+\check{O}^{32}\right),
\end{aligned}
$$




$$
\begin{aligned}
& (G \cdot O)_{34}^{++++}=G_{\mu \nu \gamma \sigma \rho \lambda}\left(\check{O}^{1}+\check{O}^{2}+\check{O}^{4}+\check{O}^{7}+\check{O}^{8}+\check{O}^{11}+\check{O}^{13}+\check{O}^{14}+\check{O}^{21}\right. \\
& \left.+\check{O}^{25}+\check{O}^{29}+\check{O}^{35}\right) \text {, } \\
& (G \cdot O)_{24}^{++++}=G_{\mu \beta \nu \sigma \rho \lambda}\left(\check{O}^{1}+\check{O}^{2}+\check{O}^{5}+\check{O}^{7}+\check{O}^{9}+\check{O}^{11}+\check{O}^{12}+\check{O}^{17}+\check{O}^{21}\right. \\
& \left.+\check{O}^{24}+\check{O}^{28}+\check{O}^{33}\right) \text {, } \\
& (G \cdot O)_{23}^{++++}=G_{\mu \beta \nu \gamma \sigma \lambda}\left(\check{O}^{1}+\check{O}^{3}+\check{O}^{5}+\check{O}^{7}+\check{O}^{8}+\check{O}^{10}+\check{O}^{12}+\check{O}^{16}+\check{O}^{20}\right. \\
& \left.+\check{O}^{24}+\check{O}^{26}+\check{O}^{36}\right) \text {, } \\
& (G \cdot O)^{++++}=G_{\mu \nu \sigma \lambda}\left(\check{O}^{1}+\check{O}^{3}+\check{O}^{4}+\check{O}^{7}+\check{O}^{9}+\check{O}^{10}+\check{O}^{13}+\check{O}^{15}+\check{O}^{20}\right. \\
& \left.+\check{O}^{25}+\check{O}^{27}+\check{O}^{37}\right) \text {, } \\
& (G \cdot O)_{1234}^{o+++}=G_{\alpha \mu \beta \nu \gamma \sigma \rho \lambda}\left(\check{O}^{46}+\check{O}^{50}+\check{O}^{58}+\check{O}^{60}\right) \\
& (G \cdot O)_{14}^{o+++}=G_{\alpha \mu \nu \sigma \rho \lambda}\left(\check{O}^{46}+\check{O}^{51}+\check{O}^{58}+\check{O}^{61}\right) \text {, } \\
& (G \cdot O)_{13}^{o+++}=G_{\alpha \mu \nu \gamma \sigma \lambda}\left(\check{O}^{47}+\check{O}^{51}+\check{O}^{58}+\check{O}^{64}\right) \text {, } \\
& (G \cdot O)_{12}^{o+++}=G_{\alpha \mu \beta \nu \sigma \lambda}\left(\check{O}^{47}+\check{O}^{50}+\check{O}^{58}+\check{O}^{62}\right) \text {, } \\
& (G \cdot O)_{34}^{o+++}=G_{\mu \nu \gamma \sigma \rho \lambda}\left(\check{O}^{49}+\check{O}^{53}+\check{O}^{59}+\check{O}^{65}\right) \text {, } \\
& (G \cdot O)_{24}^{o+++}=G_{\mu \beta \nu \sigma \rho \lambda}\left(\check{O}^{49}+\check{O}^{52}+\check{O}^{59}+\check{O}^{63}\right) \text {, } \\
& (G \cdot O)_{23}^{o+++}=G_{\mu \beta \nu \gamma \sigma \lambda}\left(\check{O}^{48}+\check{O}^{52}+\check{O}^{59}+\check{O}^{66}\right) \text {, } \\
& (G \cdot O)^{o+++}=G_{\mu \nu \sigma \lambda}\left(\check{O}^{48}+\check{O}^{53}+\check{O}^{59}+\check{O}^{67}\right) \text {, } \\
& (G \cdot O)_{1234}^{o o+++}=G_{\alpha \mu \beta \nu \gamma \sigma \rho \lambda}\left(\check{O}^{54}+\check{O}^{68}+\check{O}^{84}\right), \\
& (G \cdot O)_{14}^{o o++}=G_{\alpha \mu \nu \sigma \rho \lambda}\left(\check{O}^{55}+\check{O}^{69}+\check{O}^{85}\right), \\
& (G \cdot O)_{13}^{o o++}=G_{\alpha \mu \nu \gamma \sigma \lambda}\left(\check{O}^{55}+\check{O}^{72}+\check{O}^{88}\right), \\
& (G \cdot O)_{12}^{o o++}=G_{\alpha \mu \beta \nu \sigma \lambda}\left(\check{O}^{54}+\check{O}^{70}+\check{O}^{86}\right) \text {, } \\
& (G \cdot O)_{34}^{o o++}=G_{\mu \nu \gamma \sigma \rho \lambda}\left(\check{O}^{57}+\check{O}^{73}+\check{O}^{89}\right) \text {, } \\
& (G \cdot O)_{24}^{o o++}=G_{\mu \beta \nu \sigma \rho \lambda}\left(\check{O}^{56}+\check{O}^{71}+\check{O}^{87}\right) \text {, } \\
& (G \cdot O)_{23}^{o o++}=G_{\mu \beta \nu \gamma \sigma \lambda}\left(\check{O}^{56}+\check{O}^{74}+\check{O}^{90}\right), \\
& (G \cdot O)^{o o++}=G_{\mu \nu \sigma \lambda}\left(\check{O}^{57}+\check{O}^{75}+\check{O}^{91}\right) \text {, } \\
& (G \cdot O)_{1234}^{o o o+}=G_{\alpha \mu \beta \nu \gamma \sigma \rho \lambda} \check{O}^{76},(G \cdot O)_{14}^{o o o+}=G_{\alpha \mu \nu \sigma \rho \lambda} \check{O}^{77}, \\
& (G \cdot O)_{13}^{\text {ooot }}=G_{\alpha \mu \nu \gamma \sigma \lambda} \check{O}^{80},(G \cdot O)_{12}^{\text {ooo+ }}=G_{\alpha \mu \beta \nu \sigma \lambda} \check{O}^{78}, \\
& (G \cdot O)_{34}^{\text {ooo+ }}=G_{\mu \nu \gamma \sigma \rho \lambda} \check{O}^{81},(G \cdot O)_{24}^{\text {ooo+ }}=G_{\mu \beta \nu \sigma \rho \lambda} \check{O}^{79},
\end{aligned}
$$




$$
\begin{aligned}
& (G \cdot O)_{23}^{o o o+}=G_{\mu \beta \nu \gamma \sigma \lambda} \check{O}^{82} \quad,(G \cdot O)^{o o o+}=G_{\mu \nu \sigma \lambda} \check{O}^{83} \\
& (G \cdot O)_{1234}^{o 00 o}=G_{\alpha \mu \beta \nu \gamma \sigma \rho \lambda} \check{O}^{38},(G \cdot O)_{14}^{o o o o}=G_{\alpha \mu \nu \sigma \rho \lambda} \check{O}^{39}, \\
& (G \cdot O)_{13}^{o 0 o o}=G_{\alpha \mu \nu \gamma \sigma \lambda} \check{O}^{42},(G \cdot O)_{12}^{o 00 o}=G_{\alpha \mu \beta \nu \sigma \lambda} \check{O}^{40}, \\
& (G \cdot O)_{34}^{\text {oooo }}=G_{\mu \nu \gamma \sigma \rho \lambda} \check{O}^{43},(G \cdot O)_{24}^{\text {oooo }}=G_{\mu \beta \nu \sigma \rho \lambda} \check{O}^{41} \text {, } \\
& (G \cdot O)_{23}^{o 0 o o}=G_{\mu \beta \nu \gamma \sigma \lambda} \check{O}^{44},(G \cdot O)^{o 00 o o}=G_{\mu \nu \sigma \lambda} \check{O}^{45},
\end{aligned}
$$

where we have assumed again the notation for the traces given in eq.(B.2) and the corresponding operators introduced here are,

$$
\begin{aligned}
& \check{O}_{i j k l}^{1 \mu \nu \sigma \lambda}=e^{4} A_{\mu} A_{\nu} A_{\sigma} A_{\lambda} \delta_{i j} \delta_{j k} \delta_{k l} \delta_{l i}-e^{3} \frac{g}{2 c_{W}}\left[Z_{\mu} A_{\nu} A_{\sigma} A_{\lambda} \delta_{j k} \delta_{k l} \delta_{l i}\left(O_{L}^{\prime}+O_{R}^{\prime}\right)_{i j}\right. \\
& +A_{\mu} Z_{\nu} A_{\sigma} A_{\lambda} \delta_{i j} \delta_{k l} \delta_{l i}\left(O_{L}^{\prime}{ }_{L}+O^{\prime}{ }_{R}\right)_{j k}+A_{\mu} A_{\nu} Z_{\sigma} A_{\lambda} \delta_{i j} \delta_{j k} \delta_{l i}\left(O^{\prime}{ }_{L}+O^{\prime}{ }_{R}\right)_{k l} \\
& \left.+A_{\mu} A_{\nu} A_{\sigma} Z_{\lambda} \delta_{i j} \delta_{j k} \delta_{k l}\left(O_{L}^{\prime}+O_{R}^{\prime}\right)_{l i}\right] \text {, } \\
& \check{O}^{2(3) \mu \nu \sigma \lambda}=e^{2} \frac{g^{2}}{2 c_{W}{ }^{2}} A_{\mu} A_{\nu} Z_{\sigma} Z_{\lambda} \delta_{i j} \delta_{j k}\left(O_{L_{k l}}^{\prime} O_{L(R)_{l i}}^{\prime}+O_{R_{k l}}^{\prime} O^{\prime}{ }_{R(L)}\right) \text {, } \\
& \check{O}^{4(5) \mu \nu \sigma \lambda}=e^{2} \frac{g^{2}}{2 c_{W}{ }^{2}} A_{\mu} A_{\sigma} Z_{\nu} Z_{\lambda} \delta_{i j} \delta_{k l}\left(O_{L_{j k}}^{\prime} O_{L(R)_{l i}}^{\prime}+O_{R_{j k}}^{\prime} O_{R(L)}^{\prime}\right) \text {, } \\
& \check{O}_{i j k l}^{6(7) \mu \nu \sigma \lambda}=e^{2} \frac{g^{2}}{2 c_{W}^{2}} A_{\nu} A_{\sigma} Z_{\mu} Z_{\lambda} \delta_{j k} \delta_{k l}\left(O_{L_{i j}}^{\prime} O_{L(R)_{l i}}^{\prime}+O_{R_{i j}}^{\prime} O_{R(L)_{l i}}^{\prime}\right) \text {, } \\
& \check{O}_{i j k l}^{8(9) \mu \nu \sigma \lambda}=e^{2} \frac{g^{2}}{2 c_{W}^{2}} A_{\mu} A_{\lambda} Z_{\nu} Z_{\sigma} \delta_{i j} \delta_{l i}\left(O_{L_{j k}}^{\prime} O_{L(R)_{k l}}^{\prime}+O_{R_{j k}}^{\prime} O_{R(L)_{k l}}^{\prime}\right) \text {, } \\
& \check{O}^{10(11) \mu \nu \sigma \lambda}=e^{2} \frac{g^{2}}{2 c_{W}{ }^{2}} A_{\nu} A_{\lambda} Z_{\mu} Z_{\sigma} \delta_{j k} \delta_{l i}\left(O_{L_{i j}}^{\prime} O_{L(R)_{k l}}^{\prime}+O_{R_{i j}}^{\prime} O_{R(L)_{k l}}^{\prime}\right) \text {, } \\
& \check{O}^{12(13) \mu \nu \sigma \lambda}{ }_{i j k l}=e^{2} \frac{g^{2}}{2 c_{W}{ }^{2}} A_{\sigma} A_{\lambda} Z_{\mu} Z_{\nu} \delta_{k l} \delta_{l i}\left(O_{L_{i j}}^{\prime} O_{L(R)_{j k}}^{\prime}+O_{R_{i j}}^{\prime} O_{R(L)_{j k}}^{\prime}\right) \text {, } \\
& \check{O}_{i j k l}^{14(15) \mu \nu \sigma \lambda}=-e \frac{g^{3}}{2 c_{W}{ }^{3}} A_{\mu} Z_{\nu} Z_{\sigma} Z_{\lambda} \delta_{i j}\left(O_{L_{j k}}^{\prime} O_{L(R)_{k l}}^{\prime} O_{L_{l i}}^{\prime}+O_{R_{j k}}^{\prime} O_{R(L)_{k l}}^{\prime} O_{R_{l i}}^{\prime}\right) \text {, } \\
& \check{O}^{16(17) \mu \nu \sigma \lambda}=-e \frac{g^{3}}{2 c_{W}{ }^{3}} A_{\mu} Z_{\nu} Z_{\sigma} Z_{\lambda} \delta_{i j}\left(O_{L_{j k}}^{\prime} O_{L(R)_{k l}}^{\prime} O_{R_{l i}}^{\prime}+O_{R_{j k}}^{\prime} O_{R(L)_{k l}}^{\prime} O_{L_{l i}}^{\prime}\right) \text {, } \\
& \check{O}_{i j k l}^{18(19) \mu \nu \sigma \lambda}=-e \frac{g^{3}}{2 c_{W}{ }^{3}} A_{\nu} Z_{\mu} Z_{\sigma} Z_{\lambda} \delta_{j k}\left(O_{L_{i j}}^{\prime} O_{L(R)_{k l}}^{\prime} O_{L_{l i}}^{\prime}+O_{R_{i j}}^{\prime} O_{R(L)_{k l}}^{\prime} O_{R_{l i}}^{\prime}\right) \text {, } \\
& \check{O}^{20(21) \mu \nu \sigma \lambda} \underset{i j k l}{i j k}=-e \frac{g^{3}}{2 c_{W}{ }^{3}} A_{\nu} Z_{\mu} Z_{\sigma} Z_{\lambda} \delta_{j k}\left(O_{L_{i j}}^{\prime} O_{L(R)_{k l}}^{\prime} O_{R_{l i}}^{\prime}+O_{R_{i j}}^{\prime} O^{\prime}{ }_{R(L)_{k l}} O_{L_{l i}}^{\prime}\right) \text {, } \\
& \check{O}_{i j k l}^{22(23) \mu \nu \sigma \lambda}=-e \frac{g^{3}}{2 c_{W}{ }^{3}} A_{\sigma} Z_{\mu} Z_{\nu} Z_{\lambda} \delta_{k l}\left(O_{L_{i j}}^{\prime} O_{L(R)_{j k}}^{\prime} O_{L_{l i}}^{\prime}+O_{R_{i j}}^{\prime} O_{R(L)_{j k}}^{\prime} O_{R_{l i}}^{\prime}\right) \text {, } \\
& \check{O}^{24(25) \mu \nu \sigma \lambda} \underset{i j k l}{i j k}=-e \frac{g^{3}}{2 c_{W}{ }^{3}} A_{\sigma} Z_{\mu} Z_{\nu} Z_{\lambda} \delta_{k l}\left(O_{L_{i j}}^{\prime} O^{\prime}{ }_{L(R)_{j k}} O_{R_{l i}}^{\prime}+O_{R_{i j}}^{\prime} O^{\prime}{ }_{R(L)_{j k}} O_{L_{l i}}^{\prime}\right) \text {, } \\
& \check{O}^{26(27) \mu \nu \sigma \lambda}=-e \frac{g^{3}}{2 c_{W}{ }^{3}} A_{\lambda} Z_{\mu} Z_{\nu} Z_{\sigma} \delta_{l i}\left(O^{\prime}{ }_{L_{i j}} O^{\prime}{ }_{L(R)_{j k}} O_{L_{k l}}^{\prime}+O^{\prime}{ }_{R_{i j}} O^{\prime}{ }_{R(L)_{j k}} O_{R_{k l}}^{\prime}\right) \text {, }
\end{aligned}
$$




$$
\begin{aligned}
& \check{O}^{28(29) \mu \nu \sigma \lambda} \underset{i j k l}{i j k}=-e \frac{g^{3}}{2 c_{W}{ }^{3}} A_{\lambda} Z_{\mu} Z_{\nu} Z_{\sigma} \delta_{l i}\left(O_{L_{i j}}^{\prime} O_{L(R)_{j k}}^{\prime} O_{R_{k l}}^{\prime}+O_{R_{i j}}^{\prime} O_{R(L)_{j k}}^{\prime} O_{L_{k l} l}^{\prime}\right), \\
& \check{O}^{30(31) \mu \nu \sigma \lambda}=\frac{g^{4}}{2 c_{W}^{4}} Z_{\mu} Z_{\nu} Z_{\sigma} Z_{\lambda}\left(O_{L_{i j}}^{\prime} O_{L(R)_{j k}}^{\prime} O_{L_{k l}}^{\prime} O_{L_{l i}}^{\prime}+O_{R_{i j}}^{\prime} O_{R(L)_{j k}}^{\prime} O_{R_{k l}}^{\prime} O_{R_{l i}}^{\prime}\right) \\
& \check{O}^{32(33) \mu \nu \sigma \lambda}=\frac{g^{4}}{2 c_{W}^{4}} Z_{\mu} Z_{\nu} Z_{\sigma} Z_{\lambda}\left(O^{\prime}{ }_{i j} O^{\prime}{ }_{L_{j k}} O^{\prime}{ }_{R_{k l}} O^{\prime}{ }_{L(R)_{l i}}+O^{\prime}{ }_{R_{i j}} O_{R_{j k}}^{\prime} O^{\prime} L_{k l} O^{\prime}{ }_{R(L)_{l i}}\right) \text {, } \\
& \check{O}^{34(35) \mu \nu \sigma \lambda} \underset{i j k l}{\sin }=\frac{g^{4}}{2 c_{W}^{4}} Z_{\mu} Z_{\nu} Z_{\sigma} Z_{\lambda}\left(O_{L_{i j}}^{\prime} O_{R_{j k}}^{\prime} O_{R_{k l}}^{\prime} O_{L(R)_{l i}}^{\prime}+O_{R_{i j}}^{\prime} O_{L_{j k}}^{\prime} O_{L_{k l}}^{\prime} O_{R}^{\prime}{ }_{R(L)_{l i}}\right) \text {, }
\end{aligned}
$$

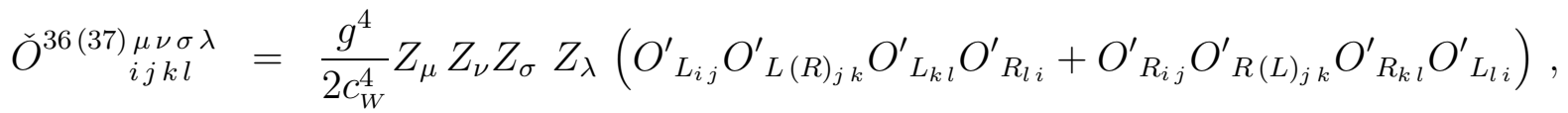

$$
\begin{aligned}
& \check{O}_{i j k l}^{38(39) \mu \nu \sigma \lambda}=\frac{g^{4}}{2 c_{W}^{4}} Z_{\mu} Z_{\nu} Z_{\sigma} Z_{\lambda}\left(O^{\prime \prime}{ }_{i j} O^{\prime \prime}{ }_{L(R)_{j k}} O^{\prime \prime}{ }_{L_{k l}} O^{\prime \prime}{ }_{l i}+O^{\prime \prime}{ }_{R_{i j}} O^{\prime \prime}{ }_{R(L) j k} O^{\prime \prime}{ }_{R_{k l}} O^{\prime \prime}{ }_{R_{l i}}\right) \text {, } \\
& \check{O}_{i j k l}^{40(41) \mu \nu \sigma \lambda}=\frac{g^{4}}{2 c_{W}^{4}} Z_{\mu} Z_{\nu} Z_{\sigma} Z_{\lambda}\left(O^{\prime \prime}{ }_{i j} O^{\prime \prime}{ }_{L_{j k}} O_{R_{k l}} O^{\prime \prime}{ }_{L(R)_{l i}}+O^{\prime \prime}{ }_{R_{i j}} O^{\prime \prime}{ }_{R_{j k}} O^{\prime \prime} L_{k l} O^{\prime \prime}{ }_{R(L)_{l i}}\right) \text {, } \\
& \check{O}^{42(43) \mu \nu \sigma \lambda} \underset{i j k l}{i j k}=\frac{g^{4}}{2 c_{W}^{4}} Z_{\mu} Z_{\nu} Z_{\sigma} Z_{\lambda}\left(O^{\prime \prime}{ }_{L_{i j}} O^{\prime \prime}{ }_{R_{j k}} O^{\prime \prime}{ }_{R_{k l}} O^{\prime \prime}{ }_{L(R)_{l i}}+O^{\prime \prime}{ }_{R_{i j}} O^{\prime \prime}{ }_{L_{j k}} O^{\prime \prime}{ }_{L_{k l}} O^{\prime \prime}{ }_{R(L)_{l i}}\right) \text {, } \\
& \check{O}^{44(45) \mu \nu \sigma \lambda} \underset{i j k l}{i j k l}=\frac{g^{4}}{2 c_{W}^{4}} Z_{\mu} Z_{\nu} Z_{\sigma} Z_{\lambda}\left(O^{\prime \prime \prime}{ }_{i j} O^{\prime \prime}{ }_{L(R)_{j k}} O^{\prime \prime}{ }_{L_{k l}} O^{\prime \prime}{ }_{R_{l i}}+O^{\prime \prime}{ }_{R_{i j}} O^{\prime \prime}{ }_{R}(L)_{j k} O^{\prime \prime}{ }_{R_{k l}} O^{\prime \prime}{ }_{l i}\right) \text {, } \\
& \check{O}^{46(47) \mu \nu \sigma \lambda}=-e \frac{g^{3}}{2 c_{W}} W_{\mu}^{-} A_{\nu} Z_{\sigma} W_{\lambda}^{+} \delta_{j k}\left(O_{L_{i j}} O_{L(R)_{k l}}^{\prime} O_{L_{l i}}^{+}+O_{R_{i j}} O_{R(L)_{k l}}^{\prime} O_{R_{l i}}^{+}\right), \\
& \check{O}_{i j k l}^{48(49) \mu \nu \sigma \lambda}=-e \frac{g^{3}}{2 c_{W}} W_{\mu}^{-} A_{\nu} Z_{\sigma} W_{\lambda}^{+} \delta_{j k}\left(O_{L_{i j}} O_{L(R)_{k l}}^{\prime} O_{R_{l i}}^{+}+O_{R_{i j}} O_{R(L)_{k l}}^{\prime} O_{L_{l i}}^{+}\right), \\
& \check{O}^{50(51) \mu \nu \sigma \lambda}=-e \frac{g^{3}}{2 c_{W}} W_{\mu}^{-} Z_{\nu} A_{\sigma} W_{\lambda}^{+} \delta_{k l}\left(O_{L_{i j}} O_{L(R)_{j k}}^{\prime} O_{L_{l i}}^{+}+O_{R_{i j}} O_{R(L)_{j k}}^{\prime} O_{R_{l i}}^{+}\right), \\
& \check{O}_{i j k l}^{52(53) \mu \nu \sigma \lambda}=-e \frac{g^{3}}{2 c_{W}} W_{\mu}^{-} Z_{\nu} A_{\sigma} W_{\lambda}^{+} \delta_{k l}\left(O_{L_{i j}} O_{L(R)_{j k}}^{\prime} O_{R_{l i}}^{+}+O_{R_{i j}} O_{R(L)_{j k}}^{\prime} O_{L_{l i}}^{+}\right) \text {, } \\
& \check{O}^{54(55) \mu \nu \sigma \lambda} \underset{i j k l}{\ln }=-e \frac{g^{3}}{2 c_{W}} W_{\nu}^{-} Z_{\mu} A_{\sigma} W_{\lambda}^{+} \delta_{k l}\left(O^{\prime \prime}{ }_{i j} O_{L(R)_{j k}} O_{L_{l i}}^{+}+O^{\prime \prime}{ }_{R_{i j}} O_{R(L)_{j k}} O_{R_{l i}}^{+}\right) \text {, } \\
& \check{O}^{56(57) \mu \nu \sigma \lambda}=-e \frac{g^{3}}{2 c_{W}} W_{\nu}^{-} Z_{\mu} A_{\sigma} W_{\lambda}^{+} \delta_{k l}\left(O^{\prime \prime}{ }_{L_{i j}} O_{L(R)_{j k}} O_{R_{l i}}^{+}+O^{\prime \prime}{ }_{R_{i j}} O_{R(L)_{j k}} O_{L_{l i}}^{+}\right) \text {, } \\
& \check{O}^{58(59) \mu \nu \sigma \lambda} \underset{i j k l}{\sin }=\frac{e^{2} g^{2}}{2} W_{\mu}^{-} A_{\nu} A_{\sigma} W_{\lambda}^{+} \delta_{j k} \delta_{k l}\left(O_{L_{i j}} O_{L(R)_{l i}}^{+}+O_{R_{i j}} O_{R(L)_{l i}}^{+}\right) \text {, } \\
& \check{O}_{i j k l}^{60(61) \mu \nu \sigma \lambda}=\frac{g^{4}}{2 c_{W}^{2}} W_{\mu}^{-} Z_{\nu} Z_{\sigma} W_{\lambda}^{+}\left(O_{L_{i j}} O_{L(R)_{j k}}^{\prime} O_{L_{k l}}^{\prime} O_{L_{l i}}^{+}+O_{R_{i j}} O_{R(L)_{j k}}^{\prime} O_{R_{k l}}^{\prime} O_{R_{l i}}^{+}\right) \text {, } \\
& \check{O}^{62(63) \mu \nu \sigma \lambda}=\frac{g^{4}}{2 c_{W}^{2}} W_{\mu}^{-} Z_{\nu} Z_{\sigma} W_{\lambda}^{+}\left(O_{L_{i j}} O_{L_{j k}}^{\prime} O_{R_{k l}}^{\prime} O_{L(R)_{l i}}^{+}+O_{R_{i j}} O_{R_{j k}}^{\prime} O_{L_{k l}}^{\prime} O_{R(L)_{l i}}^{+}\right) \text {, } \\
& \check{O}_{i j k l}^{64(65) \mu \nu \sigma \lambda}=\frac{g^{4}}{2 c_{W}^{2}} W_{\mu}^{-} Z_{\nu} Z_{\sigma} W_{\lambda}^{+}\left(O_{L_{i j}} O_{R_{j k}}^{\prime} O_{R_{k l}}^{\prime} O_{L(R)_{l i}}^{+}+O_{R_{i j}} O_{L_{j k}}^{\prime} O_{L_{k l}}^{\prime} O_{R(L)_{l i}}^{+}\right) \text {, }
\end{aligned}
$$




$$
\begin{aligned}
& \check{O}^{66(67) \mu \nu \sigma \lambda}=\frac{g^{4}}{2 c_{W}^{2}} W_{\mu}^{-} Z_{\nu} Z_{\sigma} W_{\lambda}^{+}\left(O_{L_{i j}} O_{L(R)_{j k}}^{\prime} O_{L_{k l}}^{\prime} O_{R_{l i}}^{+}+O_{R_{i j}} O^{\prime}{ }_{R(L)_{j k}} O_{R_{k l}}^{\prime} O_{L_{l i}}^{+}\right) \\
& \check{O}^{68(69) \mu \nu \sigma \lambda}=\frac{g^{4}}{2 c_{W}^{2}} W_{\nu}^{-} Z_{\mu} Z_{\sigma} W_{\lambda}^{+}\left(O^{\prime \prime}{ }_{L_{i j}} O_{L(R)_{j k}} O_{L_{k l}}^{\prime} O_{L_{l i}}^{+}+O^{\prime \prime}{ }_{R_{i j}} O_{R(L))_{j k}} O_{R_{k l}}^{\prime} O_{R_{l i}}^{+}\right) \\
& \check{O}^{70(71) \mu \nu \sigma \lambda} \underset{i j k l}{\ln k l}=\frac{g^{4}}{2 c_{W}^{2}} W_{\nu}^{-} Z_{\mu} Z_{\sigma} W_{\lambda}^{+}\left(O^{\prime \prime}{ }_{L_{i j}} O_{L_{j k}} O^{\prime}{ }_{R_{k l}} O_{L(R)_{l i}}^{+}+O^{\prime \prime}{ }_{R_{i j}} O_{R_{j k}} O^{\prime}{ }_{L_{k l}} O_{R(L)_{l i}}^{+}\right) \\
& \check{O}^{72(73) \mu \nu \sigma \lambda}=\frac{g^{4}}{2 c_{W}^{2}} W_{\nu}^{-} Z_{\mu} Z_{\sigma} W_{\lambda}^{+}\left(O^{\prime \prime}{ }_{L_{i j}} O_{R_{j k}} O_{R_{k l}}^{\prime} O_{L(R)_{l i}}^{+}+O^{\prime \prime} R_{i j} O_{L_{j k}} O_{L_{k l}}^{\prime} O_{R(L)_{l i}}^{+}\right) \text {, } \\
& \check{O}^{74(75) \mu \nu \sigma \lambda} \underset{i j k l}{\mu \nu}=\frac{g^{4}}{2 c_{W}^{2}} W_{\nu}^{-} Z_{\mu} Z_{\sigma} W_{\lambda}^{+}\left(O^{\prime \prime}{ }_{L_{i j}} O_{L(R)_{j k}} O_{L_{k l}}^{\prime} O_{R_{l i}}^{+}+O^{\prime \prime}{ }_{R_{i j}} O_{R(L)_{j k}} O_{R_{k l}}^{\prime} O_{L_{l i}}^{+}\right) \\
& \check{O}^{76(77) \mu \nu \sigma \lambda} \underset{i j k l}{i j k}=\frac{g^{4}}{2 c_{W}^{2}} W_{\sigma}^{-} Z_{\mu} Z_{\nu} W_{\lambda}^{+}\left(O^{\prime \prime}{ }_{L_{i j}} O^{\prime \prime}{ }_{L(R)_{j k}} O_{L_{k l}} O_{L_{l i}}^{+}+O^{\prime \prime}{ }_{R_{i j}} O^{\prime \prime}{ }_{R(L)_{j k}} O_{R_{k l}} O_{R_{l i}}^{+}\right) \text {, }
\end{aligned}
$$

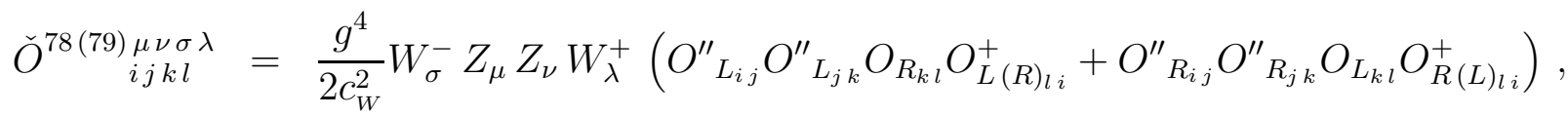

$$
\begin{aligned}
& \check{O}^{80(81) \mu \nu \sigma \lambda}=\frac{g^{4}}{2 c_{W}^{2}} W_{\sigma}^{-} Z_{\mu} Z_{\nu} W_{\lambda}^{+}\left(O_{L_{i j}}^{\prime \prime} O_{R_{j k}}^{\prime \prime} O_{R_{k l}} O_{L(R)_{l i}}^{+}+O^{\prime \prime} R_{i j} O^{\prime \prime}{ }_{L_{j k}} O_{L_{k l}} O_{R(L)_{l i}}^{+}\right) \text {, } \\
& \check{O}_{i j k l}^{82(83) \mu \nu \sigma \lambda}=\frac{g^{4}}{2 c_{W}^{2}} W_{\sigma}^{-} Z_{\mu} Z_{\nu} W_{\lambda}^{+}\left(O_{L_{i j}}^{\prime \prime} O^{\prime \prime}{ }_{L(R)_{j k}} O_{L_{k l}} O_{R_{l i}}^{+}+O_{R_{i j}}^{\prime \prime} O_{R(L)_{j k}} O_{R_{k l}} O_{L_{l i}}^{+}\right) \text {, }
\end{aligned}
$$

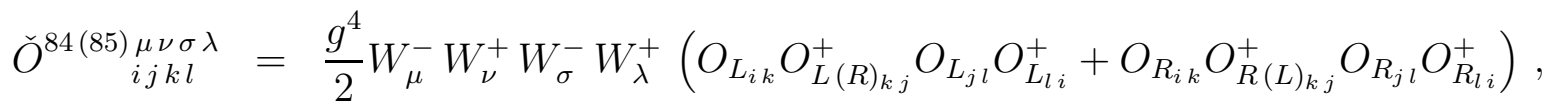

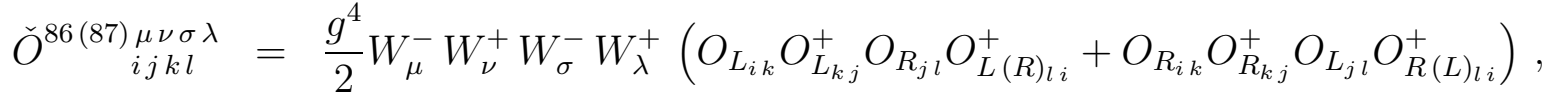

$$
\begin{aligned}
& \check{O}_{i j k l}^{88(89) \mu \nu \sigma \lambda}=\frac{g^{4}}{2} W_{\mu}^{-} W_{\nu}^{+} W_{\sigma}^{-} W_{\lambda}^{+}\left(O_{L_{i k}} O_{R_{k j}}^{+} O_{R_{j l}} O_{L(R)_{l i}}^{+}+O_{R_{i k}} O_{L_{k j}}^{+} O_{L_{j l}} O_{R(L)_{l i}}^{+}\right) \text {, } \\
& \check{O}_{i j k l}^{90(91) \mu \nu \sigma \lambda}=\frac{g^{4}}{2} W_{\mu}^{-} W_{\nu}^{+} W_{\sigma}^{-} W_{\lambda}^{+}\left(O_{L_{i k}} O_{L(R)_{k j}}^{+} O_{L_{j l}} O_{R_{l i}}^{+}+O_{R_{i k}} O_{R(L)_{k j}}^{+} O_{R_{j l}} O_{L_{l i}}^{+}\right) \text {, }
\end{aligned}
$$

The definitions of the coupling matrices, $\hat{Q}_{f}, \hat{G}_{f}, \Sigma_{f}^{t b}, \Sigma_{f}^{b t}, \Sigma_{f}, O_{L, R}, O_{L, R}^{\prime}$ and $O_{L, R}^{\prime \prime}$ of the above equations can be found in [16].

Finally, we give explicitely in the following the expressions for the $f_{i}$ and $g_{i}$ functions:

$$
\begin{aligned}
f_{1} & =-2 c_{b}^{2} c_{t}^{2} \log \frac{2 \tilde{m}_{t_{1}}^{2}+\tilde{m}_{b_{1}}^{2}}{3 \mu_{o}^{2}}-2 s_{b}^{2} c_{t}^{2} \log \frac{2 \tilde{m}_{t_{1}}^{2}+\tilde{m}_{b_{2}}^{2}}{3 \mu_{o}^{2}}-2 c_{b}^{2} s_{t}^{2} \log \frac{2 \tilde{m}_{t_{2}}^{2}+\tilde{m}_{b_{1}}^{2}}{3 \mu_{o}^{2}} \\
& -2 s_{b}^{2} s_{t}^{2} \log \frac{2 \tilde{m}_{t_{2}}^{2}+\tilde{m}_{b_{2}}^{2}}{3 \mu_{o}^{2}}+c_{b}^{2} c_{t}^{2} \log \frac{\tilde{m}_{t_{1}}^{2}+2 \tilde{m}_{b_{1}}^{2}}{3 \mu_{o}^{2}}+s_{b}^{2} c_{t}^{2} \log \frac{\tilde{m}_{t_{1}}^{2}+2 \tilde{m}_{b_{2}}^{2}}{3 \mu_{o}^{2}} \\
& +c_{b}^{2} s_{t}^{2} \log \frac{\tilde{m}_{t_{2}}^{2}+2 \tilde{m}_{b_{1}}^{2}}{3 \mu_{o}^{2}}+s_{b}^{2} s_{t}^{2} \log \frac{\tilde{m}_{t_{2}}^{2}+2 \tilde{m}_{b_{2}}^{2}}{3 \mu_{o}^{2}},
\end{aligned}
$$




$$
\begin{aligned}
& f_{2}=c_{b}^{2} c_{t}^{2}\left[\left(\frac{c_{t}^{2}}{2}-\frac{2}{3} s_{W}^{2}\right) \log \frac{2 \tilde{m}_{t_{1}}^{2}+\tilde{m}_{b_{1}}^{2}}{3 \mu_{o}^{2}}+\left(-\frac{c_{b}^{2}}{2}+\frac{1}{3} s_{W}^{2}\right) \log \frac{\tilde{m}_{t_{1}}^{2}+2 \tilde{m}_{b_{1}}^{2}}{3 \mu_{o}^{2}}\right] \\
& +s_{b}^{2} s_{t}^{2}\left[\left(\frac{s_{t}^{2}}{2}-\frac{2}{3} s_{W}^{2}\right) \log \frac{2 \tilde{m}_{t_{2}}^{2}+\tilde{m}_{b_{2}}^{2}}{3 \mu_{o}^{2}}+\left(-\frac{s_{b}^{2}}{2}+\frac{1}{3} s_{W}^{2}\right) \log \frac{\tilde{m}_{t_{2}}^{2}+2 \tilde{m}_{b_{2}}^{2}}{3 \mu_{o}^{2}}\right] \\
& +s_{b}^{2} c_{t}^{2}\left[\left(\frac{c_{t}^{2}}{2}-\frac{2}{3} s_{W}^{2}\right) \log \frac{2 \tilde{m}_{t_{1}}^{2}+\tilde{m}_{b_{2}}^{2}}{3 \mu_{o}^{2}}+\left(-\frac{s_{b}^{2}}{2}+\frac{1}{3} s_{W}^{2}\right) \log \frac{\tilde{m}_{t_{1}}^{2}+2 \tilde{m}_{b_{2}}^{2}}{3 \mu_{o}^{2}}\right] \\
& +c_{b}^{2} s_{t}^{2}\left[\left(\frac{s_{t}^{2}}{2}-\frac{2}{3} s_{W}^{2}\right) \log \frac{2 \tilde{m}_{t_{2}}^{2}+\tilde{m}_{b_{1}}^{2}}{3 \mu_{o}^{2}}+\left(-\frac{c_{b}^{2}}{2}+\frac{1}{3} s_{W}^{2}\right) \log \frac{\tilde{m}_{t_{2}}^{2}+2 \tilde{m}_{b_{1}}^{2}}{3 \mu_{o}^{2}}\right] \\
& +c_{b}^{2} c_{t}^{2} s_{t}^{2} \log \frac{\tilde{m}_{t_{1}}^{2}+\tilde{m}_{t_{2}}^{2}+\tilde{m}_{b_{1}}^{2}}{3 \mu_{o}^{2}}+s_{b}^{2} c_{t}^{2} s_{t}^{2} \log \frac{\tilde{m}_{t_{1}}^{2}+\tilde{m}_{t_{2}}^{2}+\tilde{m}_{b_{2}}^{2}}{3 \mu_{o}^{2}} \\
& -c_{b}^{2} s_{b}^{2} c_{t}^{2} \log \frac{\tilde{m}_{t_{1}}^{2}+\tilde{m}_{b_{1}}^{2}+\tilde{m}_{b_{2}}^{2}}{3 \mu_{o}^{2}}-c_{b}^{2} s_{b}^{2} s_{t}^{2} \log \frac{\tilde{m}_{t_{2}}^{2}+\tilde{m}_{b_{1}}^{2}+\tilde{m}_{b_{2}}^{2}}{3 \mu_{o}^{2}},
\end{aligned}
$$

$$
\begin{aligned}
f_{3} & =-\log \frac{2 \tilde{M}_{1}^{+2}+\tilde{M}_{2}^{o 2}}{3 \mu_{o}^{2}}-\frac{1}{4} \log \frac{2 \tilde{M}_{2}^{+2}+\tilde{M}_{3}^{o 2}}{3 \mu_{o}^{2}}-\frac{1}{4} \log \frac{2 \tilde{M}_{2}^{+2}+\tilde{M}_{4}^{o 2}}{3 \mu_{o}^{2}} \\
f_{4} & =-\left(s_{W}^{2}-1\right) \log \frac{2 \tilde{M}_{1}^{+2}+\tilde{M}_{2}^{o 2}}{3 \mu_{o}^{2}}-\frac{1}{8}\left(2 s_{W}^{2}-1\right)\left(\log \frac{2 \tilde{M}_{2}^{+2}+\tilde{M}_{3}^{o 2}}{3 \mu_{o}^{2}}+\log \frac{2 \tilde{M}_{2}^{+2}+\tilde{M}_{4}^{o 2}}{3 \mu_{o}^{2}}\right) \\
& +\frac{1}{4} \log \frac{\tilde{M}_{2}^{+2}+\tilde{M}_{3}^{o 2}+\tilde{M}_{4}^{o 2}}{3 \mu_{o}^{2}}
\end{aligned}
$$

and,

$$
\begin{aligned}
& g_{1}=-\frac{2}{3}\left\{c _ { b } ^ { 2 } c _ { t } ^ { 2 } \left[\log \frac{\tilde{m}_{t_{1}}^{2}+2 \tilde{m}_{b_{1}}^{2}}{3 \mu_{o}^{2}}+4 \log \frac{2 \tilde{m}_{t_{1}}^{2}+\tilde{m}_{b_{1}}^{2}}{3 \mu_{o}^{2}}-\frac{8}{3} \log \frac{3 \tilde{m}_{t_{1}}^{2}+\tilde{m}_{b_{1}}^{2}}{4 \mu_{o}^{2}}-\frac{2}{3} \log \frac{\tilde{m}_{t_{1}}^{2}+3 \tilde{m}_{b_{1}}^{2}}{4 \mu_{o}^{2}}\right.\right. \\
& \left.+\frac{4}{3} \log \frac{\tilde{m}_{t_{1}}^{2}+\tilde{m}_{b_{1}}^{2}}{2 \mu_{o}^{2}}\right]+s_{b}^{2} c_{t}^{2}\left[\log \frac{\tilde{m}_{t_{1}}^{2}+2 \tilde{m}_{b_{2}}^{2}}{3 \mu_{o}^{2}}+4 \log \frac{2 \tilde{m}_{t_{1}}^{2}+\tilde{m}_{b_{2}}^{2}}{3 \mu_{o}^{2}}-\frac{8}{3} \log \frac{3 \tilde{m}_{t_{1}}^{2}+\tilde{m}_{b_{2}}^{2}}{4 \mu_{o}^{2}}\right. \\
& \left.-\frac{2}{3} \log \frac{\tilde{m}_{t_{1}}^{2}+3 \tilde{m}_{b_{2}}^{2}}{4 \mu_{o}^{2}}+\frac{4}{3} \log \frac{\tilde{m}_{t_{1}}^{2}+\tilde{m}_{b_{2}}^{2}}{2 \mu_{o}^{2}}\right]+c_{b}^{2} s_{t}^{2}\left[\log \frac{\tilde{m}_{t_{2}}^{2}+2 \tilde{m}_{b_{1}}^{2}}{3 \mu_{o}^{2}}+4 \log \frac{2 \tilde{m}_{t_{2}}^{2}+\tilde{m}_{b_{1}}^{2}}{3 \mu_{o}^{2}}\right. \\
& \left.-\frac{8}{3} \log \frac{3 \tilde{m}_{t_{2}}^{2}+\tilde{m}_{b_{1}}^{2}}{4 \mu_{o}^{2}}-\frac{2}{3} \log \frac{\tilde{m}_{t_{2}}^{2}+3 \tilde{m}_{b_{1}}^{2}}{4 \mu_{o}^{2}}+\frac{4}{3} \log \frac{\tilde{m}_{t_{2}}^{2}+\tilde{m}_{b_{1}}^{2}}{2 \mu_{o}^{2}}\right]+s_{b}^{2} s_{t}^{2}\left[\log \frac{\tilde{m}_{t_{2}}^{2}+2 \tilde{m}_{b_{2}}^{2}}{3 \mu_{o}^{2}}\right. \\
& \left.\left.+4 \log \frac{2 \tilde{m}_{t_{2}}^{2}+\tilde{m}_{b_{2}}^{2}}{3 \mu_{o}^{2}}-\frac{8}{3} \log \frac{3 \tilde{m}_{t_{2}}^{2}+\tilde{m}_{b_{2}}^{2}}{4 \mu_{o}^{2}}-\frac{2}{3} \log \frac{\tilde{m}_{t_{2}}^{2}+3 \tilde{m}_{b_{2}}^{2}}{4 \mu_{o}^{2}}+\frac{4}{3} \log \frac{\tilde{m}_{t_{2}}^{2}+\tilde{m}_{b_{2}}^{2}}{2 \mu_{o}^{2}}\right]\right\} \\
& g_{2}=-\frac{2}{3}\left\{c _ { b } ^ { 2 } c _ { t } ^ { 2 } \left[-\frac{1}{2} \log \frac{\tilde{m}_{t_{1}}^{2}+\tilde{m}_{b_{1}}^{2}}{2 \mu_{o}^{2}}-\log \frac{\tilde{m}_{t_{1}}^{2}+2 \tilde{m}_{b_{1}}^{2}}{3 \mu_{o}^{2}}+2 \log \frac{2 \tilde{m}_{t_{1}}^{2}+\tilde{m}_{b_{1}}^{2}}{3 \mu_{o}^{2}}-\frac{8}{3} \log \frac{3 \tilde{m}_{t_{1}}^{2}+\tilde{m}_{b_{1}}^{2}}{4 \mu_{o}^{2}}\right.\right. \\
& \left.-\frac{2}{3} \log \frac{\tilde{m}_{t_{1}}^{2}+3 \tilde{m}_{b_{1}}^{2}}{4 \mu_{o}^{2}}+\frac{4}{3} \log \frac{\tilde{m}_{t_{1}}^{2}+\tilde{m}_{b_{1}}^{2}}{2 \mu_{o}^{2}}\right]+s_{b}^{2} c_{t}^{2}\left[\frac{1}{2} \log \frac{\tilde{m}_{t_{1}}^{2}+\tilde{m}_{b_{2}}^{2}}{2 \mu_{o}^{2}}+\log \frac{\tilde{m}_{t_{1}}^{2}+2 \tilde{m}_{b_{2}}^{2}}{3 \mu_{o}^{2}}\right. \\
& \left.-2 \log \frac{2 \tilde{m}_{t_{1}}^{2}+\tilde{m}_{b_{2}}^{2}}{3 \mu_{o}^{2}}+\frac{8}{3} \log \frac{3 \tilde{m}_{t_{1}}^{2}+\tilde{m}_{b_{2}}^{2}}{4 \mu_{o}^{2}}+\frac{2}{3} \log \frac{\tilde{m}_{t_{1}}^{2}+3 \tilde{m}_{b_{2}}^{2}}{4 \mu_{o}^{2}}-\frac{4}{3} \log \frac{\tilde{m}_{t_{1}}^{2}+\tilde{m}_{b_{2}}^{2}}{2 \mu_{o}^{2}}\right]
\end{aligned}
$$




$$
\begin{aligned}
& +c_{b}^{2} s_{t}^{2}\left[-\frac{1}{2} \log \frac{\tilde{m}_{t_{2}}^{2}+\tilde{m}_{b_{1}}^{2}}{\mu_{o}^{2}}-\log \frac{\tilde{m}_{t_{2}}^{2}+2 \tilde{m}_{b_{1}}^{2}}{3 \mu_{o}^{2}}+2 \log \frac{2 \tilde{m}_{t_{2}}^{2}+\tilde{m}_{b_{1}}^{2}}{3 \mu_{o}^{2}}-\frac{8}{3} \log \frac{3 \tilde{m}_{t_{2}}^{2}+\tilde{m}_{b_{1}}^{2}}{4 \mu_{o}^{2}}\right. \\
& \left.-\frac{2}{3} \log \frac{\tilde{m}_{t_{2}}^{2}+3 \tilde{m}_{b_{1}}^{2}}{4 \mu_{o}^{2}}+\frac{4}{3} \log \frac{\tilde{m}_{t_{2}}^{2}+\tilde{m}_{b_{1}}^{2}}{2 \mu_{o}^{2}}\right]+s_{b}^{2} s_{t}^{2}\left[-\frac{1}{2} \log \frac{\tilde{m}_{t_{2}}^{2}+\tilde{m}_{b_{2}}^{2}}{2 \mu_{o}^{2}}-\log \frac{\tilde{m}_{t_{2}}^{2}+2 \tilde{m}_{b_{2}}^{2}}{3 \mu_{o}^{2}}\right. \\
& \left.\left.+2 \log \frac{2 \tilde{m}_{t_{2}}^{2}+\tilde{m}_{b_{2}}^{2}}{3 \mu_{o}^{2}}-\frac{8}{3} \log \frac{3 \tilde{m}_{t_{2}}^{2}+\tilde{m}_{b_{2}}^{2}}{4 \mu_{o}^{2}}-\frac{2}{3} \log \frac{\tilde{m}_{t_{2}}^{2}+3 \tilde{m}_{b_{2}}^{2}}{4 \mu_{o}^{2}}+\frac{4}{3} \log \frac{\tilde{m}_{t_{2}}^{2}+\tilde{m}_{b_{2}}^{2}}{2 \mu_{o}^{2}}\right]\right\} \\
& g_{3}=c_{t}^{2} s_{t}^{2}\left[4 \log \frac{\tilde{m}_{t_{1}}^{2}+\tilde{m}_{t_{2}}^{2}}{2 \mu_{o}^{2}}-2 \log \frac{2 \tilde{m}_{t_{1}}^{2}+\tilde{m}_{t_{2}}^{2}}{3 \mu_{o}^{2}}-2 \log \frac{\tilde{m}_{t_{1}}^{2}+2 \tilde{m}_{t_{2}}^{2}}{3 \mu_{o}^{2}}\right] \\
& +c_{b}^{2} c_{t}^{2}\left[\frac{2}{3}\left(-3 c_{t}^{2}+4 s_{W}^{2}\right) \log \frac{2 \tilde{m}_{t_{1}}^{2}+\tilde{m}_{b_{1}}^{2}}{3 \mu_{o}^{2}}+\frac{1}{3}\left(-3 c_{b}^{2}+2 s_{W}^{2}\right) \log \frac{\tilde{m}_{t_{1}}^{2}+2 \tilde{m}_{b_{1}}^{2}}{3 \mu_{o}^{2}}\right. \\
& +\frac{1}{9}\left(-6 c_{b}^{2}-3 c_{t}^{2}+8 s_{W}^{2}\right) \log \frac{\tilde{m}_{t_{1}}^{2}+\tilde{m}_{b_{1}}^{2}}{2 \mu_{o}^{2}}+\frac{4}{9}\left(3 c_{t}^{2}-4 s_{W}^{2}\right) \log \frac{3 \tilde{m}_{t_{1}}^{2}+\tilde{m}_{b_{1}}^{2}}{4 \mu_{o}^{2}} \\
& \left.+\frac{4}{9}\left(3 c_{b}^{2}-2 s_{W}^{2}\right) \log \frac{\tilde{m}_{t_{1}}^{2}+3 \tilde{m}_{b_{1}}^{2}}{4 \mu_{o}^{2}}\right]+s_{b}^{2} c_{t}^{2}\left[\frac{2}{3}\left(-3 c_{t}^{2}+4 s_{W}^{2}\right) \log \frac{2 \tilde{m}_{t_{1}}^{2}+\tilde{m}_{b_{2}}^{2}}{3 \mu_{o}^{2}}\right. \\
& +\frac{1}{3}\left(-3 s_{b}^{2}+2 s_{W}^{2}\right) \log \frac{\tilde{m}_{t_{1}}^{2}+2 \tilde{m}_{b_{2}}^{2}}{3 \mu_{o}^{2}}+\frac{1}{9}\left(-6 s_{b}^{2}-3 c_{t}^{2}+8 s_{W}^{2}\right) \log \frac{\tilde{m}_{t_{1}}^{2}+\tilde{m}_{b_{2}}^{2}}{2 \mu_{o}^{2}} \\
& \left.+\frac{4}{9}\left(3 c_{t}^{2}-4 s_{W}^{2}\right) \log \frac{3 \tilde{m}_{t_{1}}^{2}+\tilde{m}_{b_{2}}^{2}}{4 \mu_{o}^{2}}+\frac{2}{9}\left(3 s_{b}^{2}-2 s_{W}^{2}\right) \log \frac{\tilde{m}_{t_{1}}^{2}+3 \tilde{m}_{b_{2}}^{2}}{4 \mu_{o}^{2}}\right] \\
& +c_{b}^{2} s_{t}^{2}\left[\frac{2}{3}\left(-3 s_{t}^{2}+4 s_{W}^{2}\right) \log \frac{2 \tilde{m}_{t_{2}}^{2}+\tilde{m}_{b_{1}}^{2}}{3 \mu_{o}^{2}}+\frac{1}{3}\left(-3 c_{b}^{2}+2 s_{W}^{2}\right) \log \frac{\tilde{m}_{t_{2}}^{2}+2 \tilde{m}_{b_{1}}^{2}}{3 \mu_{o}^{2}}\right. \\
& +\frac{1}{9}\left(-6 c_{b}^{2}-3 s_{t}^{2}+8 s_{W}^{2}\right) \log \frac{\tilde{m}_{t_{2}}^{2}+\tilde{m}_{b_{1}}^{2}}{2 \mu_{o}^{2}}+\frac{4}{9}\left(3 s_{t}^{2}-4 s_{W}^{2}\right) \log \frac{3 \tilde{m}_{t_{2}}^{2}+\tilde{m}_{b_{1}}^{2}}{4 \mu_{o}^{2}} \\
& \left.+\frac{2}{9}\left(3 c_{b}^{2}-2 s_{W}^{2}\right) \log \frac{\tilde{m}_{t_{2}}^{2}+3 \tilde{m}_{b_{1}}^{2}}{4 \mu_{o}^{2}}\right]+s_{b}^{2} s_{t}^{2}\left[\frac{2}{3}\left(-3 s_{t}^{2}+4 s_{W}^{2}\right) \log \frac{2 \tilde{m}_{t_{2}}^{2}+\tilde{m}_{b_{2}}^{2}}{3 \mu_{o}^{2}}\right. \\
& +\frac{1}{3}\left(-3 s_{b}^{2}+2 s_{W}^{2}\right) \log \frac{\tilde{m}_{t_{2}}^{2}+2 \tilde{m}_{b_{2}}^{2}}{3 \mu_{o}^{2}}+\frac{1}{9}\left(-6 s_{b}^{2}-3 s_{t}^{2}+8 s_{W}^{2}\right) \log \frac{\tilde{m}_{t_{2}}^{2}+\tilde{m}_{b_{2}}^{2}}{2 \mu_{o}^{2}} \\
& \left.+\frac{4}{9}\left(3 s_{t}^{2}-4 s_{W}^{2}\right) \log \frac{3 \tilde{m}_{t_{2}}^{2}+\tilde{m}_{b_{2}}^{2}}{4 \mu_{o}^{2}}+\frac{2}{9}\left(3 s_{b}^{2}-2 s_{W}^{2}\right) \log \frac{\tilde{m}_{t_{2}}^{2}+3 \tilde{m}_{b_{2}}^{2}}{4 \mu_{o}^{2}}\right] \\
& +c_{b}^{2} s_{b}^{2}\left[2 \log \frac{\tilde{m}_{b_{1}}^{2}+\tilde{m}_{b_{2}}^{2}}{2 \mu_{o}^{2}}-\log \frac{2 \tilde{m}_{b_{1}}^{2}+\tilde{m}_{b_{2}}^{2}}{3 \mu_{o}^{2}}-\log \frac{\tilde{m}_{b_{1}}^{2}+2 \tilde{m}_{b_{2}}^{2}}{3 \mu_{o}^{2}}\right] \\
& +c_{b}^{2} c_{t}^{2} s_{t}^{2}\left[-4 \log \frac{\tilde{m}_{t_{1}}^{2}+\tilde{m}_{t_{2}}^{2}+\tilde{m}_{b_{1}}^{2}}{3 \mu_{o}^{2}}+\frac{4}{3} \log \frac{\tilde{m}_{t_{1}}^{2}+2 \tilde{m}_{t_{2}}^{2}+\tilde{m}_{b_{1}}^{2}}{4 \mu_{o}^{2}}+\frac{4}{3} \log \frac{2 \tilde{m}_{t_{1}}^{2}+\tilde{m}_{t_{2}}^{2}+\tilde{m}_{b_{1}}^{2}}{4 \mu_{o}^{2}}\right. \\
& \left.-\frac{2}{3} \log \frac{\tilde{m}_{t_{1}}^{2}+\tilde{m}_{t_{2}}^{2}+2 \tilde{m}_{b_{1}}^{2}}{4 \mu_{o}^{2}}\right]+s_{b}^{2} c_{t}^{2} s_{t}^{2}\left[-4 \log \frac{\tilde{m}_{t_{1}}^{2}+\tilde{m}_{t_{2}}^{2}+\tilde{m}_{b_{2}}^{2}}{3 \mu_{o}^{2}}+\frac{4}{3} \log \frac{2 \tilde{m}_{t_{1}}^{2}+\tilde{m}_{t_{2}}^{2}+\tilde{m}_{b_{2}}^{2}}{4 \mu_{o}^{2}}\right. \\
& \left.+\frac{4}{3} \log \frac{\tilde{m}_{t_{1}}^{2}+2 \tilde{m}_{t_{2}}^{2}+\tilde{m}_{b_{2}}^{2}}{4 \mu_{o}^{2}}-\frac{2}{3} \log \frac{\tilde{m}_{t_{1}}^{2}+\tilde{m}_{t_{2}}^{2}+2 \tilde{m}_{b_{2}}^{2}}{4 \mu_{o}^{2}}\right]+s_{b}^{2} c_{b}^{2} c_{t}^{2}\left[-2 \log \frac{\tilde{m}_{t_{1}}^{2}+\tilde{m}_{b_{1}}^{2}+\tilde{m}_{b_{2}}^{2}}{3 \mu_{o}^{2}}\right.
\end{aligned}
$$




$$
\begin{aligned}
& \left.-\frac{4}{3} \log \frac{2 \tilde{m}_{t_{1}}^{2}+\tilde{m}_{b_{1}}^{2}+\tilde{m}_{b_{2}}^{2}}{4 \mu_{o}^{2}}+\frac{2}{3} \log \frac{\tilde{m}_{t_{1}}^{2}+2 \tilde{m}_{b_{1}}^{2}+\tilde{m}_{b_{2}}^{2}}{4 \mu_{o}^{2}}+\frac{2}{3} \log \frac{\tilde{m}_{t_{1}}^{2}+\tilde{m}_{b_{1}}^{2}+2 \tilde{m}_{b_{2}}^{2}}{4 \mu_{o}^{2}}\right] \\
& +s_{b}^{2} c_{b}^{2} s_{t}^{2}\left[-2 \log \frac{\tilde{m}_{t_{2}}^{2}+\tilde{m}_{b_{1}}^{2}+\tilde{m}_{b_{2}}^{2}}{3 \mu_{o}^{2}}+\frac{2}{3} \log \frac{\tilde{m}_{t_{2}}^{2}+2 \tilde{m}_{b_{1}}^{2}+\tilde{m}_{b_{2}}^{2}}{4 \mu_{o}^{2}}+\frac{2}{3} \log \frac{\tilde{m}_{t_{2}}^{2}+\tilde{m}_{b_{1}}^{2}+2 \tilde{m}_{b_{2}}^{2}}{4 \mu_{o}^{2}}\right. \\
& \left.\left.-\frac{4}{3} \log \frac{2 \tilde{m}_{t_{2}}^{2}+\tilde{m}_{b_{1}}^{2}+\tilde{m}_{b_{2}}^{2}}{4 \mu_{o}^{2}}\right]\right\} \\
& g_{4}=c_{b}^{2} c_{t}^{2}\left[\frac{1}{6}\left(-3 c_{t}^{2}+8 s_{W}^{2}\right) \log \frac{2 \tilde{m}_{t_{1}}^{2}+\tilde{m}_{b_{1}}^{2}}{3 \mu_{o}^{2}}+\frac{1}{6}\left(3 c_{b}^{2}-4 s_{W}^{2}\right) \log \frac{\tilde{m}_{t_{1}}^{2}+2 \tilde{m}_{b_{1}}^{2}}{3 \mu_{o}^{2}}\right. \\
& +\frac{1}{9}\left(-6 c_{b}^{2}-3 c_{t}^{2}+5 s_{W}^{2}\right) \log \frac{\tilde{m}_{t_{1}}^{2}+\tilde{m}_{b_{1}}^{2}}{2 \mu_{o}^{2}}+\frac{4}{9}\left(3 c_{t}^{2}-4 s_{W}^{2}\right) \log \frac{3 \tilde{m}_{t_{1}}^{2}+\tilde{m}_{b_{1}}^{2}}{4 \mu_{o}^{2}} \\
& \left.+\frac{2}{9}\left(3 c_{b}^{2}-2 s_{W}^{2}\right) \log \frac{\tilde{m}_{t_{1}}^{2}+3 \tilde{m}_{b_{1}}^{2}}{4 \mu_{o}^{2}}\right]+s_{b}^{2} c_{t}^{2}\left[\frac{1}{6}\left(-3 c_{t}^{2}+8 s_{W}^{2}\right) \log \frac{2 \tilde{m}_{t_{1}}^{2}+\tilde{m}_{b_{2}}^{2}}{3 \mu_{o}^{2}}\right. \\
& +\frac{1}{6}\left(3 s_{b}^{2}-4 s_{W}^{2}\right) \log \frac{\tilde{m}_{t_{1}}^{2}+2 \tilde{m}_{b_{2}}^{2}}{3 \mu_{o}^{2}}+\frac{1}{9}\left(-6 s_{b}^{2}-3 c_{t}^{2}+5 s_{W}^{2}\right) \log \frac{\tilde{m}_{t_{1}}^{2}+\tilde{m}_{b_{2}}^{2}}{2 \mu_{o}^{2}} \\
& \left.+\frac{4}{9}\left(3 c_{t}^{2}-4 s_{W}^{2}\right) \log \frac{3 \tilde{m}_{t_{1}}^{2}+\tilde{m}_{b_{2}}^{2}}{4 \mu_{o}^{2}}+\frac{2}{9}\left(3 s_{b}^{2}-2 s_{W}^{2}\right) \log \frac{\tilde{m}_{t_{1}}^{2}+3 \tilde{m}_{b_{2}}^{2}}{4 \mu_{o}^{2}}\right] \\
& +c_{b}^{2} s_{t}^{2}\left[\frac{1}{6}\left(-3 s_{t}^{2}+8 s_{W}^{2}\right) \log \frac{2 \tilde{m}_{t_{2}}^{2}+\tilde{m}_{b_{1}}^{2}}{3 \mu_{o}^{2}}+\frac{1}{6}\left(3 c_{b}^{2}-4 s_{W}^{2}\right) \log \frac{\tilde{m}_{t_{2}}^{2}+2 \tilde{m}_{b_{1}}^{2}}{3 \mu_{o}^{2}}\right. \\
& +\frac{1}{9}\left(-6 c_{b}^{2}-3 s_{t}^{2}+5 s_{W}^{2}\right) \log \frac{\tilde{m}_{t_{2}}^{2}+\tilde{m}_{b_{1}}^{2}}{2 \mu_{o}^{2}}+\frac{4}{9}\left(3 s_{t}^{2}-4 s_{W}^{2}\right) \log \frac{3 \tilde{m}_{t_{2}}^{2}+\tilde{m}_{b_{1}}^{2}}{4 \mu_{o}^{2}} \\
& \left.+\frac{2}{9}\left(3 c_{b}^{2}-2 s_{W}^{2}\right) \log \frac{\tilde{m}_{t_{2}}^{2}+3 \tilde{m}_{b_{1}}^{2}}{4 \mu_{o}^{2}}\right]+s_{b}^{2} s_{t}^{2}\left[\frac{1}{6}\left(-3 s_{t}^{2}+8 s_{W}^{2}\right) \log \frac{2 \tilde{m}_{t_{2}}^{2}+\tilde{m}_{b_{2}}^{2}}{3 \mu_{o}^{2}}\right. \\
& +\frac{1}{6}\left(3 s_{b}^{2}-4 s_{W}^{2}\right) \log \frac{\tilde{m}_{t_{2}}^{2}+2 \tilde{m}_{b_{2}}^{2}}{3 \mu_{o}^{2}}+\frac{1}{9}\left(-6 s_{b}^{2}-3 s_{t}^{2}+5 s_{W}^{2}\right) \log \frac{\tilde{m}_{t_{2}}^{2}+\tilde{m}_{b_{2}}^{2}}{2 \mu_{o}^{2}} \\
& \left.+\frac{4}{9}\left(3 s_{t}^{2}-4 s_{W}^{2}\right) \log \frac{3 \tilde{m}_{t_{2}}^{2}+\tilde{m}_{b_{2}}^{2}}{4 \mu_{o}^{2}}+\frac{2}{9}\left(3 s_{b}^{2}-2 s_{W}^{2}\right) \log \frac{\tilde{m}_{t_{2}}^{2}+3 \tilde{m}_{b_{2}}^{2}}{4 \mu_{o}^{2}}\right] \\
& +c_{b}^{2} c_{t}^{2} s_{t}^{2}\left[-\log \frac{\tilde{m}_{t_{1}}^{2}+\tilde{m}_{t_{2}}^{2}+\tilde{m}_{b_{1}}^{2}}{3 \mu_{o}^{2}}+\frac{4}{3} \log \frac{2 \tilde{m}_{t_{1}}^{2}+\tilde{m}_{t_{2}}^{2}+\tilde{m}_{b_{1}}^{2}}{4 \mu_{o}^{2}}+\frac{4}{3} \log \frac{\tilde{m}_{t_{1}}^{2}+2 \tilde{m}_{t_{2}}^{2}+\tilde{m}_{b_{1}}^{2}}{4 \mu_{o}^{2}}\right. \\
& \left.-\frac{2}{3} \log \frac{\tilde{m}_{t_{1}}^{2}+\tilde{m}_{t_{2}}^{2}+2 \tilde{m}_{b_{1}}^{2}}{4 \mu_{o}^{2}}\right]+s_{b}^{2} c_{t}^{2} s_{t}^{2}\left[-\log \frac{\tilde{m}_{t_{1}}^{2}+\tilde{m}_{t_{2}}^{2}+\tilde{m}_{b_{2}}^{2}}{3 \mu_{o}^{2}}+\frac{4}{3} \log \frac{2 \tilde{m}_{t_{1}}^{2}+\tilde{m}_{t_{2}}^{2}+\tilde{m}_{b_{2}}^{2}}{4 \mu_{o}^{2}}\right. \\
& \left.+\frac{4}{3} \log \frac{\tilde{m}_{t_{1}}^{2}+2 \tilde{m}_{t_{2}}^{2}+\tilde{m}_{b_{2}}^{2}}{4 \mu_{o}^{2}}-\frac{2}{3} \log \frac{\tilde{m}_{t_{1}}^{2}+\tilde{m}_{t_{2}}^{2}+2 \tilde{m}_{b_{2}}^{2}}{4 \mu_{o}^{2}}\right]+s_{b}^{2} c_{b}^{2} c_{t}^{2}\left[\log \frac{\tilde{m}_{t_{1}}^{2}+\tilde{m}_{b_{1}}^{2}+\tilde{m}_{b_{2}}^{2}}{3 \mu_{o}^{2}}\right. \\
& \left.-\frac{4}{3} \log \frac{2 \tilde{m}_{t_{1}}^{2}+\tilde{m}_{b_{1}}^{2}+\tilde{m}_{b_{2}}^{2}}{4 \mu_{o}^{2}}+\frac{2}{3} \log \frac{\tilde{m}_{t_{1}}^{2}+2 \tilde{m}_{b_{1}}^{2}+\tilde{m}_{b_{2}}^{2}}{4 \mu_{o}^{2}}+\frac{2}{3} \log \frac{\tilde{m}_{t_{1}}^{2}+\tilde{m}_{b_{1}}^{2}+2 \tilde{m}_{b_{2}}^{2}}{4 \mu_{o}^{2}}\right] \\
& +s_{b}^{2} c_{b}^{2} s_{t}^{2}\left[\log \frac{\tilde{m}_{t_{2}}^{2}+\tilde{m}_{b_{1}}^{2}+\tilde{m}_{b_{2}}^{2}}{3 \mu_{o}^{2}}+\frac{2}{3} \log \frac{\tilde{m}_{t_{2}}^{2}+2 \tilde{m}_{b_{1}}^{2}+\tilde{m}_{b_{2}}^{2}}{4 \mu_{o}^{2}}+\frac{2}{3} \log \frac{\tilde{m}_{t_{2}}^{2}+\tilde{m}_{b_{1}}^{2}+2 \tilde{m}_{b_{2}}^{2}}{4 \mu_{o}^{2}}\right.
\end{aligned}
$$




$$
\left.\left.-\frac{4}{3} \log \frac{2 \tilde{m}_{t_{2}}^{2}+\tilde{m}_{b_{1}}^{2}+\tilde{m}_{b_{2}}^{2}}{4 \mu_{o}^{2}}\right]\right\}
$$

$g_{5}=\frac{3}{2} s_{t}^{2} c_{t}^{4} \log \frac{\tilde{m}_{t_{1}}^{2}}{\mu_{o}^{2}}+\frac{3}{2} s_{t}^{4} c_{t}^{2} \log \frac{\tilde{m}_{t_{2}}^{2}}{\mu_{o}^{2}}+\frac{3}{2} c_{b}^{4} s_{b}^{2} \log \frac{\tilde{m}_{b_{1}}^{2}}{\mu_{o}^{2}}+\frac{3}{2} c_{b}^{2} s_{b}^{4} \log \frac{\tilde{m}_{b_{2}}^{2}}{\mu_{o}^{2}}$

$-c_{b}^{2} c_{t}^{2}\left[\left(\frac{3}{2} s_{t}^{2} c_{t}^{2}+6\left(\frac{c_{t}^{2}}{2}-\frac{2}{3} s_{W}^{2}\right)^{2}\right) \log \frac{2 \tilde{m}_{t_{1}}^{2}+\tilde{m}_{b_{1}}^{2}}{3 \mu_{o}^{2}}-4\left(\frac{c_{t}^{2}}{2}-\frac{2}{3} s_{W}^{2}\right)^{2} \log \frac{3 \tilde{m}_{t_{1}}^{2}+\tilde{m}_{b_{1}}^{2}}{4 \mu_{o}^{2}}\right.$

$+\left(\frac{3}{2} s_{b}^{2} c_{b}^{2}+\left(-\frac{c_{b}^{2}}{2}+\frac{1}{3} s_{W}^{2}\right)^{2}\right) \log \frac{\tilde{m}_{t_{1}}^{2}+2 \tilde{m}_{b_{1}}^{2}}{3 \mu_{o}^{2}}-4\left(-\frac{c_{b}^{2}}{2}+\frac{1}{3} s_{W}^{2}\right)^{2} \log \frac{\tilde{m}_{t_{1}}^{2}+3 \tilde{m}_{b_{1}}^{2}}{4 \mu_{o}^{2}}$

$\left.-\frac{1}{9}\left(3 c_{t}^{2}-4 s_{W}^{2}\right)\left(-3 c_{b}^{2}+2 s_{W}^{2}\right) \log \frac{\tilde{m}_{t_{1}}^{2}+\tilde{m}_{b_{1}}^{2}}{2 \mu_{o}^{2}}\right]-s_{b}^{2} s_{t}^{2}\left[-4\left(\frac{s_{t}^{2}}{2}-\frac{2}{3} s_{W}^{2}\right)^{2} \log \frac{3 \tilde{m}_{t_{2}}^{2}+\tilde{m}_{b_{2}}^{2}}{4 \mu_{o}^{2}}\right.$

$+\left(\frac{3}{2} s_{t}^{2} c_{t}^{2}+6\left(\frac{s_{t}^{2}}{2}-\frac{2}{3} s_{W}^{2}\right)^{2}\right) \log \frac{2 \tilde{m}_{t_{2}}^{2}+\tilde{m}_{b_{2}}^{2}}{3 \mu_{o}^{2}}+\left(\frac{3}{2} s_{b}^{2} c_{b}^{2}+6\left(-\frac{s_{b}^{2}}{2}+\frac{1}{3} s_{W}^{2}\right)^{2}\right) \log \frac{\tilde{m}_{t_{2}}^{2}+2 \tilde{m}_{b_{2}}^{2}}{3 \mu_{o}^{2}}$

$\left.-4\left(-\frac{s_{b}^{2}}{2}+\frac{1}{3} s_{W}^{2}\right)^{2} \log \frac{\tilde{m}_{t_{2}}^{2}+3 \tilde{m}_{b_{2}}^{2}}{4 \mu_{o}^{2}}-\frac{1}{9}\left(3 s_{t}^{2}-4 s_{W}^{2}\right)\left(-3 s_{b}^{2}+2 s_{W}^{2}\right) \log \frac{\tilde{m}_{t_{2}}^{2}+\tilde{m}_{b_{2}}^{2}}{2 \mu_{o}^{2}}\right]$

$-s_{b}^{2} c_{t}^{2}\left[\left(\frac{3}{2} s_{t}^{2} c_{t}^{2}+6\left(\frac{c_{t}^{2}}{2}-\frac{2}{3} s_{W}^{2}\right)^{2}\right) \log \frac{2 \tilde{m}_{t_{1}}^{2}+\tilde{m}_{b_{2}}^{2}}{3 \mu_{o}^{2}}-4\left(\frac{c_{t}^{2}}{2}-\frac{2}{3} s_{W}^{2}\right)^{2} \log \frac{3 \tilde{m}_{t_{1}}^{2}+\tilde{m}_{b_{2}}^{2}}{4 \mu_{o}^{2}}\right.$

$+\left(\frac{3}{2} s_{b}^{2} c_{b}^{2}+6\left(-\frac{s_{b}^{2}}{2}+\frac{1}{3} s_{W}^{2}\right)^{2}\right) \log \frac{\tilde{m}_{t_{1}}^{2}+2 \tilde{m}_{b_{2}}^{2}}{3 \mu_{o}^{2}}-4\left(-\frac{s_{b}^{2}}{2}+\frac{1}{3} s_{W}^{2}\right)^{2} \log \frac{\tilde{m}_{t_{1}}^{2}+3 \tilde{m}_{b_{2}}^{2}}{4 \mu_{o}^{2}}$

$\left.-\frac{1}{9}\left(3 c_{t}^{2}-4 s_{W}^{2}\right)\left(-3 s_{b}^{2}+2 s_{W}^{2}\right) \log \frac{\tilde{m}_{t_{1}}^{2}+\tilde{m}_{b_{2}}^{2}}{2 \mu_{o}^{2}}\right]-c_{b}^{2} s_{t}^{2}\left[-4\left(\frac{s_{t}^{2}}{2}-\frac{2}{3} s_{W}^{2}\right)^{2} \log \frac{3 \tilde{m}_{t_{2}}^{2}+\tilde{m}_{b_{1}}^{2}}{4 \mu_{o}^{2}}\right.$

$+\left(\frac{3}{2} s_{t}^{2} c_{t}^{2}+6\left(\frac{s_{t}^{2}}{2}-\frac{2}{3} s_{W}^{2}\right)^{2}\right) \log \frac{2 \tilde{m}_{t_{2}}^{2}+\tilde{m}_{b_{1}}^{2}}{3 \mu_{o}^{2}}+\left(\frac{3}{2} s_{b}^{2} c_{b}^{2}+6\left(-\frac{c_{b}^{2}}{2}+\frac{1}{3} s_{W}^{2}\right)^{2}\right) \log \frac{\tilde{m}_{t_{2}}^{2}+2 \tilde{m}_{b_{1}}^{2}}{3 \mu_{o}^{2}}$

$\left.-4\left(-\frac{c_{b}^{2}}{2}+\frac{1}{3} s_{W}^{2}\right)^{2} \log \frac{\tilde{m}_{t_{2}}^{2}+3 \tilde{m}_{b_{1}}^{2}}{4 \mu_{o}^{2}}-\frac{1}{9}\left(3 s_{t}^{2}-4 s_{W}^{2}\right)\left(-3 c_{b}^{2}+2 s_{W}^{2}\right) \log \frac{\tilde{m}_{t_{2}}^{2}+\tilde{m}_{b_{1}}^{2}}{2 \mu_{o}^{2}}\right]$

$-s_{t}^{2} c_{t}^{2}\left[-\left(3-8 s_{W}^{2}\right) \log \frac{\tilde{m}_{t_{1}}^{2}+\tilde{m}_{t_{2}}^{2}}{2 \mu_{o}^{2}}+\frac{1}{2}\left(9 c_{t}^{2}-8 s_{W}^{2}\right) \log \frac{2 \tilde{m}_{t_{1}}^{2}+\tilde{m}_{t_{2}}^{2}}{3 \mu_{o}^{2}}\right.$

$\left.+\frac{1}{2}\left(9 s_{t}^{2}-8 s_{W}^{2}\right) \log \frac{\tilde{m}_{t_{1}}^{2}+2 \tilde{m}_{t_{2}}^{2}}{3 \mu_{o}^{2}}\right]-s_{b}^{2} c_{b}^{2}\left[-\left(3-4 s_{W}^{2}\right) \log \frac{\tilde{m}_{b_{1}}^{2}+\tilde{m}_{b_{2}}^{2}}{2 \mu_{o}^{2}}\right.$

$\left.+\frac{1}{2}\left(9 c_{b}^{2}-4 s_{W}^{2}\right) \log \frac{2 \tilde{m}_{b_{1}}^{2}+\tilde{m}_{b_{2}}^{2}}{3 \mu_{o}^{2}}+\frac{1}{2}\left(9 s_{b}^{2}-4 s_{W}^{2}\right) \log \frac{\tilde{m}_{b_{1}}^{2}+2 \tilde{m}_{b_{2}}^{2}}{3 \mu_{o}^{2}}\right]$

$+4 c_{b}^{2} c_{t}^{2} s_{t}^{2}\left(-\frac{c_{b}^{2}}{2}+\frac{1}{3} s_{W}^{2}\right) \log \frac{\tilde{m}_{t_{1}}^{2}+\tilde{m}_{t_{2}}^{2}+2 \tilde{m}_{b_{1}}^{2}}{4 \mu_{o}^{2}}+4 s_{b}^{2} c_{t}^{2} s_{t}^{2}\left(-\frac{s_{b}^{2}}{2}+\frac{1}{3} s_{W}^{2}\right) \log \frac{\tilde{m}_{t_{1}}^{2}+\tilde{m}_{t_{2}}^{2}+2 \tilde{m}_{b_{2}}^{2}}{4 \mu_{o}^{2}}$

$-4 c_{b}^{2} c_{t}^{2} s_{b}^{2}\left(\frac{c_{t}^{2}}{2}-\frac{2}{3} s_{W}^{2}\right) \log \frac{2 \tilde{m}_{t_{1}}^{2}+\tilde{m}_{b_{1}}^{2}+\tilde{m}_{b_{2}}^{2}}{4 \mu_{o}^{2}}-4 s_{b}^{2} c_{b}^{2} s_{t}^{2}\left(\frac{s_{t}^{2}}{2}-\frac{2}{3} s_{W}^{2}\right) \log \frac{2 \tilde{m}_{t_{2}}^{2}+\tilde{m}_{b_{1}}^{2}+\tilde{m}_{b_{2}}^{2}}{4 \mu_{o}^{2}}$ 


$$
\begin{aligned}
& +\frac{1}{3} c_{b}^{2} s_{t}^{2} c_{t}^{2}\left[\left(9 c_{t}^{2}-8 s_{W}^{2}\right) \log \frac{2 \tilde{m}_{t_{1}}^{2}+\tilde{m}_{t_{2}}^{2}+\tilde{m}_{b_{1}}^{2}}{4 \mu_{o}^{2}}+\left(9 s_{t}^{2}-8 s_{W}^{2}\right) \log \frac{\tilde{m}_{t_{1}}^{2}+2 \tilde{m}_{t_{2}}^{2}+\tilde{m}_{b_{1}}^{2}}{4 \mu_{o}^{2}}\right. \\
& \left.-3\left(3-8 s_{W}^{2}\right) \log \frac{\tilde{m}_{t_{1}}^{2}+\tilde{m}_{t_{2}}^{2}+\tilde{m}_{b_{1}}^{2}}{3 \mu_{o}^{2}}\right]+\frac{1}{3} s_{b}^{2} s_{t}^{2} c_{t}^{2}\left[\left(9 c_{t}^{2}-8 s_{W}^{2}\right) \log \frac{2 \tilde{m}_{t_{1}}^{2}+\tilde{m}_{t_{2}}^{2}+\tilde{m}_{b_{2}}^{2}}{4 \mu_{o}^{2}}\right. \\
& \left.+\left(9 s_{t}^{2}-8 s_{W}^{2}\right) \log \frac{\tilde{m}_{t_{1}}^{2}+2 \tilde{m}_{t_{2}}^{2}+\tilde{m}_{b_{2}}^{2}}{4 \mu_{o}^{2}}-3\left(3-8 s_{W}^{2}\right) \log \frac{\tilde{m}_{t_{1}}^{2}+\tilde{m}_{t_{2}}^{2}+\tilde{m}_{b_{2}}^{2}}{3 \mu_{o}^{2}}\right] \\
& +\frac{1}{3} c_{b}^{2} s_{b}^{2} c_{t}^{2}\left[\left(9 c_{b}^{2}-4 s_{W}^{2}\right) \log \frac{\tilde{m}_{t_{1}}^{2}+2 \tilde{m}_{b_{1}}^{2}+\tilde{m}_{b_{2}}^{2}}{4 \mu_{o}^{2}}+\left(9 s_{b}^{2}-4 s_{W}^{2}\right) \log \frac{\tilde{m}_{t_{1}}^{2}+\tilde{m}_{b_{1}}^{2}+2 \tilde{m}_{b_{2}}^{2}}{4 \mu_{o}^{2}}\right. \\
& \left.-3\left(3-4 s_{W}^{2}\right) \log \frac{\tilde{m}_{t_{1}}^{2}+\tilde{m}_{b_{1}}^{2}+\tilde{m}_{b_{2}}^{2}}{3 \mu_{o}^{2}}\right]+\frac{1}{3} c_{b}^{2} s_{b}^{2} s_{t}^{2}\left[\left(9 c_{b}^{2}-4 s_{W}^{2}\right) \log \frac{\tilde{m}_{t_{2}}^{2}+2 \tilde{m}_{b_{1}}^{2}+\tilde{m}_{b_{2}}^{2}}{4 \mu_{o}^{2}}\right. \\
& \left.+\left(9 s_{b}^{2}-4 s_{W}^{2}\right) \log \frac{\tilde{m}_{t_{2}}^{2}+\tilde{m}_{b_{1}}^{2}+2 \tilde{m}_{b_{2}}^{2}}{4 \mu_{o}^{2}}-3\left(3-4 s_{W}^{2}\right) \log \frac{\tilde{m}_{t_{2}}^{2}+\tilde{m}_{b_{1}}^{2}+\tilde{m}_{b_{2}}^{2}}{3 \mu_{o}^{2}}\right] \\
& -4 c_{b}^{2} c_{t}^{2} s_{b}^{2} s_{t}^{2} \log \frac{\tilde{m}_{t_{1}}^{2}+\tilde{m}_{t_{2}}^{2}+\tilde{m}_{b_{1}}^{2}+\tilde{m}_{b_{2}}^{2}}{4 \mu_{o}^{2}} \\
& g_{6}=c_{b}^{2} c_{t}^{2}\left[\frac{s_{W}^{4}}{3} \log \frac{\tilde{m}_{t_{1}}^{2}+\tilde{m}_{b_{1}}^{2}}{2 \mu_{o}^{2}}+\frac{s_{W}^{2}}{3}\left(3 c_{t}^{2}-4 s_{W}^{2}\right) \log \frac{2 \tilde{m}_{t_{1}}^{2}+\tilde{m}_{b_{1}}^{2}}{3 \mu_{o}^{2}}\right. \\
& +2 s_{W}^{2}\left(-\frac{c_{b}^{2}}{2}+\frac{s_{W}^{2}}{3}\right) \log \frac{\tilde{m}_{t_{1}}^{2}+2 \tilde{m}_{b_{1}}^{2}}{3 \mu_{o}^{2}}+\frac{1}{9}\left(3 c_{t}^{2}-4 s_{W}^{2}\right)\left(-3 c_{b}^{2}+2 s_{W}^{2}\right) \log \frac{\tilde{m}_{t_{1}}^{2}+\tilde{m}_{b_{1}}^{2}}{2 \mu_{o}^{2}} \\
& \left.+4\left(\frac{c_{t}^{2}}{2}-\frac{2}{3} s_{W}^{2}\right)^{2} \log \frac{3 \tilde{m}_{t_{1}}^{2}+\tilde{m}_{b_{1}}^{2}}{4 \mu_{o}^{2}}+4\left(-\frac{c_{b}^{2}}{2}+\frac{s_{W}^{2}}{3}\right)^{2} \log \frac{\tilde{m}_{t_{1}}^{2}+3 \tilde{m}_{b_{1}}^{2}}{4 \mu_{o}^{2}}\right] \\
& +c_{b}^{2} s_{t}^{2}\left[\frac{s_{W}^{4}}{3} \log \frac{\tilde{m}_{t_{2}}^{2}+\tilde{m}_{b_{1}}^{2}}{2 \mu_{o}^{2}}+\frac{s_{W}^{2}}{3}\left(3 s_{t}^{2}-4 s_{W}^{2}\right) \log \frac{2 \tilde{m}_{t_{2}}^{2}+\tilde{m}_{b_{1}}^{2}}{3 \mu_{o}^{2}}\right. \\
& +2 s_{W}^{2}\left(-\frac{c_{b}^{2}}{2}+\frac{s_{W}^{2}}{3}\right) \log \frac{\tilde{m}_{t_{2}}^{2}+2 \tilde{m}_{b_{1}}^{2}}{3 \mu_{o}^{2}}+\frac{1}{9}\left(3 s_{t}^{2}-4 s_{W}^{2}\right)\left(-3 c_{b}^{2}+2 s_{W}^{2}\right) \log \frac{\tilde{m}_{t_{2}}^{2}+\tilde{m}_{b_{1}}^{2}}{2 \mu_{o}^{2}} \\
& \left.+4\left(\frac{s_{t}^{2}}{2}-\frac{2}{3} s_{W}^{2}\right)^{2} \log \frac{3 \tilde{m}_{t_{2}}^{2}+\tilde{m}_{b_{1}}^{2}}{4 \mu_{o}^{2}}+4\left(-\frac{c_{b}^{2}}{2}+\frac{s_{W}^{2}}{3}\right)^{2} \log \frac{\tilde{m}_{t_{2}}^{2}+3 \tilde{m}_{b_{1}}^{2}}{4 \mu_{o}^{2}}\right] \\
& +s_{b}^{2} c_{t}^{2}\left[\frac{s_{W}^{4}}{3} \log \frac{\tilde{m}_{t_{1}}^{2}+\tilde{m}_{b_{2}}^{2}}{2 \mu_{o}^{2}}+\frac{s_{W}^{2}}{3}\left(3 c_{t}^{2}-4 s_{W}^{2}\right) \log \frac{2 \tilde{m}_{t_{1}}^{2}+\tilde{m}_{b_{2}}^{2}}{3 \mu_{o}^{2}}\right. \\
& +2 s_{W}^{2}\left(-\frac{s_{b}^{2}}{2}+\frac{s_{W}^{2}}{3}\right) \log \frac{\tilde{m}_{t_{1}}^{2}+2 \tilde{m}_{b_{2}}^{2}}{3 \mu_{o}^{2}}+\frac{1}{9}\left(3 c_{t}^{2}-4 s_{W}^{2}\right)\left(-3 s_{b}^{2}+2 s_{W}^{2}\right) \log \frac{\tilde{m}_{t_{1}}^{2}+\tilde{m}_{b_{2}}^{2}}{2 \mu_{o}^{2}} \\
& \left.+4\left(\frac{c_{t}^{2}}{2}-\frac{2}{3} s_{W}^{2}\right)^{2} \log \frac{3 \tilde{m}_{t_{1}}^{2}+\tilde{m}_{b_{2}}^{2}}{4 \mu_{o}^{2}}+4\left(-\frac{s_{b}^{2}}{2}+\frac{s_{W}^{2}}{3}\right)^{2} \log \frac{\tilde{m}_{t_{1}}^{2}+3 \tilde{m}_{b_{2}}^{2}}{4 \mu_{o}^{2}}\right] \\
& +s_{b}^{2} s_{t}^{2}\left[\frac{s_{W}^{4}}{3} \log \frac{\tilde{m}_{t_{2}}^{2}+\tilde{m}_{b_{2}}^{2}}{2 \mu_{o}^{2}}+\frac{s_{W}^{2}}{3}\left(3 s_{t}^{2}-4 s_{W}^{2}\right) \log \frac{2 \tilde{m}_{t_{2}}^{2}+\tilde{m}_{b_{2}}^{2}}{3 \mu_{o}^{2}}\right. \\
& +2 s_{W}^{2}\left(-\frac{s_{b}^{2}}{2}+\frac{s_{W}^{2}}{3}\right) \log \frac{\tilde{m}_{t_{2}}^{2}+2 \tilde{m}_{b_{2}}^{2}}{3 \mu_{o}^{2}}+\frac{1}{9}\left(3 s_{t}^{2}-4 s_{W}^{2}\right)\left(-3 s_{b}^{2}+2 s_{W}^{2}\right) \log \frac{\tilde{m}_{t_{2}}^{2}+\tilde{m}_{b_{2}}^{2}}{2 \mu_{o}^{2}}
\end{aligned}
$$




$$
\begin{aligned}
& \left.+4\left(\frac{s_{t}^{2}}{2}-\frac{2}{3} s_{W}^{2}\right)^{2} \log \frac{3 \tilde{m}_{t_{2}}^{2}+\tilde{m}_{b_{2}}^{2}}{4 \mu_{o}^{2}}+4\left(-\frac{s_{b}^{2}}{2}+\frac{s_{W}^{2}}{3}\right)^{2} \log \frac{\tilde{m}_{t_{2}}^{2}+3 \tilde{m}_{b_{2}}^{2}}{4 \mu_{o}^{2}}\right] \\
& +4 c_{b}^{2} c_{t}^{2} s_{t}^{2}\left(-\frac{c_{b}^{2}}{2}+\frac{s_{W}^{2}}{3}\right) \log \frac{\tilde{m}_{t_{1}}^{2}+\tilde{m}_{t_{2}}^{2}+2 \tilde{m}_{b_{1}}^{2}}{4 \mu_{o}^{2}}+4 s_{b}^{2} c_{t}^{2} s_{t}^{2}\left(-\frac{s_{b}^{2}}{2}+\frac{s_{W}^{2}}{3}\right) \log \frac{\tilde{m}_{t_{1}}^{2}+\tilde{m}_{t_{2}}^{2}+2 \tilde{m}_{b_{2}}^{2}}{4 \mu_{o}^{2}} \\
& -4 c_{b}^{2} c_{t}^{2} s_{b}^{2}\left(\frac{c_{t}^{2}}{2}-\frac{2}{3} s_{W}^{2}\right) \log \frac{2 \tilde{m}_{t_{1}}^{2}+\tilde{m}_{b_{1}}^{2}+\tilde{m}_{b_{2}}^{2}}{4 \mu_{o}^{2}}-4 s_{b}^{2} c_{b}^{2} s_{t}^{2}\left(\frac{s_{t}^{2}}{2}-\frac{2}{3} s_{W}^{2}\right) \log \frac{2 \tilde{m}_{t_{2}}^{2}+\tilde{m}_{b_{1}}^{2}+\tilde{m}_{b_{2}}^{2}}{4 \mu_{o}^{2}} \\
& +\frac{1}{3} c_{b}^{2} s_{t}^{2} c_{t}^{2}\left[6 s_{W}^{2} \log \frac{\tilde{m}_{t_{1}}^{2}+\tilde{m}_{t_{2}}^{2}+\tilde{m}_{b_{1}}^{2}}{3 \mu_{o}^{2}}+\left(9 c_{t}^{2}-8 s_{W}^{2}\right) \log \frac{2 \tilde{m}_{t_{1}}^{2}+\tilde{m}_{t_{2}}^{2}+\tilde{m}_{b_{1}}^{2}}{4 \mu_{o}^{2}}\right. \\
& \left.+\left(9 s_{t}^{2}-8 s_{W}^{2}\right) \log \frac{\tilde{m}_{t_{1}}^{2}+2 \tilde{m}_{t_{2}}^{2}+\tilde{m}_{b_{1}}^{2}}{4 \mu_{o}^{2}}\right]+\frac{1}{3} s_{b}^{2} s_{t}^{2} c_{t}^{2}\left[6 s_{W}^{2} \log \frac{\tilde{m}_{t_{1}}^{2}+\tilde{m}_{t_{2}}^{2}+\tilde{m}_{b_{2}}^{2}}{3 \mu_{o}^{2}}\right. \\
& \left.+\left(9 c_{t}^{2}-8 s_{W}^{2}\right) \log \frac{2 \tilde{m}_{t_{1}}^{2}+\tilde{m}_{t_{2}}^{2}+\tilde{m}_{b_{2}}^{2}}{4 \mu_{o}^{2}}+\left(9 s_{t}^{2}-8 s_{W}^{2}\right) \log \frac{\tilde{m}_{t_{1}}^{2}+2 \tilde{m}_{t_{2}}^{2}+\tilde{m}_{b_{2}}^{2}}{4 \mu_{o}^{2}}\right] \\
& +\frac{1}{3} c_{b}^{2} s_{b}^{2} c_{t}^{2}\left[-6 s_{W}^{2} \log \frac{\tilde{m}_{t_{1}}^{2}+\tilde{m}_{b_{1}}^{2}+\tilde{m}_{b_{2}}^{2}}{3 \mu_{o}^{2}}+\left(9 c_{b}^{2}-4 s_{W}^{2}\right) \log \frac{\tilde{m}_{t_{1}}^{2}+2 \tilde{m}_{b_{1}}^{2}+\tilde{m}_{b_{2}}^{2}}{4 \mu_{o}^{2}}\right. \\
& \left.+\left(9 s_{b}^{2}-4 s_{W}^{2}\right) \log \frac{\tilde{m}_{t_{1}}^{2}+\tilde{m}_{b_{1}}^{2}+2 \tilde{m}_{b_{2}}^{2}}{4 \mu_{o}^{2}}\right]+\frac{1}{3} c_{b}^{2} s_{b}^{2} s_{t}^{2}\left[-6 s_{W}^{2} \log \frac{\tilde{m}_{t_{2}}^{2}+\tilde{m}_{b_{1}}^{2}+\tilde{m}_{b_{2}}^{2}}{3 \mu_{o}^{2}}\right. \\
& \left.+\left(9 c_{b}^{2}-4 s_{W}^{2}\right) \log \frac{\tilde{m}_{t_{2}}^{2}+2 \tilde{m}_{b_{1}}^{2}+\tilde{m}_{b_{2}}^{2}}{4 \mu_{o}^{2}}+\left(9 s_{b}^{2}-4 s_{W}^{2}\right) \log \frac{\tilde{m}_{t_{2}}^{2}+\tilde{m}_{b_{1}}^{2}+2 \tilde{m}_{b_{2}}^{2}}{4 \mu_{o}^{2}}\right] \\
& -4 c_{b}^{2} c_{t}^{2} s_{b}^{2} s_{t}^{2} \log \frac{\tilde{m}_{t_{1}}^{2}+\tilde{m}_{t_{2}}^{2}+\tilde{m}_{b_{1}}^{2}+\tilde{m}_{b_{2}}^{2}}{4 \mu_{o}^{2}} \\
& g_{7}=\frac{3}{2} c_{t}^{4} \log \frac{\tilde{m}_{t_{1}}^{2}}{\mu_{o}^{2}}+\frac{3}{2} s_{t}^{4} \log \frac{\tilde{m}_{t_{2}}^{2}}{\mu_{o}^{2}}+\frac{3}{2} c_{b}^{4} \log \frac{\tilde{m}_{b_{1}}^{2}}{\mu_{o}^{2}}+\frac{3}{2} s_{b}^{4} \log \frac{\tilde{m}_{b_{2}}^{2}}{\mu_{o}^{2}}+3 s_{t}^{2} c_{t}^{2} \log \frac{\tilde{m}_{t_{1}}^{2}+\tilde{m}_{t_{2}}^{2}}{2 \mu_{o}^{2}} \\
& +c_{t}^{4} c_{b}^{4} \log \frac{\tilde{m}_{t_{1}}^{2}+\tilde{m}_{b_{1}}^{2}}{2 \mu_{o}^{2}}+s_{t}^{4} c_{b}^{4} \log \frac{\tilde{m}_{t_{2}}^{2}+\tilde{m}_{b_{1}}^{2}}{2 \mu_{o}^{2}}-3 c_{b}^{2} c_{t}^{4} \log \frac{2 \tilde{m}_{t_{1}}^{2}+\tilde{m}_{b_{1}}^{2}}{3 \mu_{o}^{2}}-3 s_{t}^{4} c_{b}^{2} \log \frac{2 \tilde{m}_{t_{2}}^{2}+\tilde{m}_{b_{1}}^{2}}{3 \mu_{o}^{2}} \\
& -3 c_{t}^{2} c_{b}^{4} \log \frac{\tilde{m}_{t_{1}}^{2}+2 \tilde{m}_{b_{1}}^{2}}{3 \mu_{o}^{2}}-3 s_{t}^{2} c_{b}^{4} \log \frac{\tilde{m}_{t_{2}}^{2}+2 \tilde{m}_{b_{1}}^{2}}{3 \mu_{o}^{2}}-6 c_{b}^{2} s_{t}^{2} c_{t}^{2} \log \frac{\tilde{m}_{t_{1}}^{2}+\tilde{m}_{t_{2}}^{2}+\tilde{m}_{b_{1}}^{2}}{3 \mu_{o}^{2}} \\
& +2 c_{b}^{4} s_{t}^{2} c_{t}^{2} \log \frac{\tilde{m}_{t_{1}}^{2}+\tilde{m}_{t_{2}}^{2}+2 \tilde{m}_{b_{1}}^{2}}{4 \mu_{o}^{2}}+c_{t}^{4} s_{b}^{4} \log \frac{\tilde{m}_{t_{1}}^{2}+\tilde{m}_{b_{2}}^{2}}{2 \mu_{o}^{2}}-3 c_{t}^{4} s_{b}^{2} \log \frac{2 \tilde{m}_{t_{1}}^{2}+\tilde{m}_{b_{2}}^{2}}{3 \mu_{o}^{2}} \\
& +s_{t}^{4} s_{b}^{4} \log \frac{\tilde{m}_{t_{2}}^{2}+\tilde{m}_{b_{2}}^{2}}{2 \mu_{o}^{2}}-3 s_{t}^{4} s_{b}^{2} \log \frac{2 \tilde{m}_{t_{2}}^{2}+\tilde{m}_{b_{2}}^{2}}{3 \mu_{o}^{2}}-6 c_{t}^{2} s_{t}^{2} s_{b}^{2} \log \frac{\tilde{m}_{t_{1}}^{2}+\tilde{m}_{t_{2}}^{2}+\tilde{m}_{b_{2}}^{2}}{3 \mu_{o}^{2}} \\
& +3 c_{b}^{2} s_{b}^{2} \log \frac{\tilde{m}_{b_{1}}^{2}+\tilde{m}_{b_{2}}^{2}}{2 \mu_{o}^{2}}-3 c_{t}^{2} s_{b}^{4} \log \frac{\tilde{m}_{t_{1}}^{2}+2 \tilde{m}_{b_{2}}^{2}}{3 \mu_{o}^{2}}-3 s_{b}^{4} s_{t}^{2} \log \frac{\tilde{m}_{t_{2}}^{2}+2 \tilde{m}_{b_{2}}^{2}}{3 \mu_{o}^{2}} \\
& -6 c_{b}^{2} s_{b}^{2} c_{t}^{2} \log \frac{\tilde{m}_{t_{1}}^{2}+\tilde{m}_{b_{1}}^{2}+\tilde{m}_{b_{2}}^{2}}{3 \mu_{o}^{2}}-6 c_{b}^{2} s_{b}^{2} s_{t}^{2} \log \frac{\tilde{m}_{t_{2}}^{2}+\tilde{m}_{b_{1}}^{2}+\tilde{m}_{b_{2}}^{2}}{3 \mu_{o}^{2}} \\
& +2 c_{b}^{2} s_{b}^{2} c_{t}^{4} \log \frac{2 \tilde{m}_{t_{1}}^{2}+\tilde{m}_{b_{1}}^{2}+\tilde{m}_{b_{2}}^{2}}{4 \mu_{o}^{2}}+2 c_{b}^{2} s_{b}^{2} s_{t}^{4} \log \frac{2 \tilde{m}_{t_{2}}^{2}+\tilde{m}_{b_{1}}^{2}+\tilde{m}_{b_{2}}^{2}}{4 \mu_{o}^{2}}
\end{aligned}
$$




$$
\begin{aligned}
& +2 c_{t}^{2} s_{t}^{2} s_{b}^{4} \log \frac{\tilde{m}_{t_{1}}^{2}+\tilde{m}_{t_{2}}^{2}+2 \tilde{m}_{b_{2}}^{2}}{4 \mu_{o}^{2}}+4 c_{b}^{2} s_{b}^{2} c_{t}^{2} s_{t}^{2} \log \frac{\tilde{m}_{t_{1}}^{2}+\tilde{m}_{t_{2}}^{2}+\tilde{m}_{b_{1}}^{2}+\tilde{m}_{b_{2}}^{2}}{4 \mu_{o}^{2}} \\
& g_{8}=c_{t}^{4} c_{b}^{4} \log \frac{\tilde{m}_{t_{1}}^{2}+\tilde{m}_{b_{1}}^{2}}{2 \mu_{o}^{2}}+s_{t}^{4} c_{b}^{4} \log \frac{\tilde{m}_{t_{2}}^{2}+\tilde{m}_{b_{1}}^{2}}{2 \mu_{o}^{2}}+s_{b}^{4} c_{t}^{4} \log \frac{\tilde{m}_{t_{1}}^{2}+\tilde{m}_{b_{2}}^{2}}{2 \mu_{o}^{2}}+s_{t}^{4} s_{b}^{4} \log \frac{\tilde{m}_{t_{2}}^{2}+\tilde{m}_{b_{2}}^{2}}{4 \mu_{o}^{2}} \\
& +2 c_{b}^{4} s_{t}^{2} c_{t}^{2} \log \frac{\tilde{m}_{t_{1}}^{2}+\tilde{m}_{t_{2}}^{2}+2 \tilde{m}_{b_{1}}^{2}}{4 \mu_{o}^{2}}+2 c_{t}^{2} s_{t}^{2} s_{b}^{4} \log \frac{\tilde{m}_{t_{1}}^{2}+\tilde{m}_{t_{2}}^{2}+2 \tilde{m}_{b_{2}}^{2}}{4 \mu_{o}^{2}} \\
& +2 c_{b}^{2} s_{b}^{2} c_{t}^{4} \log \frac{2 \tilde{m}_{t_{1}}^{2}+\tilde{m}_{b_{1}}^{2}+\tilde{m}_{b_{2}}^{2}}{4 \mu_{o}^{2}}+2 c_{b}^{2} s_{b}^{2} s_{t}^{4} \log \frac{2 \tilde{m}_{t_{2}}^{2}+\tilde{m}_{b_{1}}^{2}+\tilde{m}_{b_{2}}^{2}}{4 \mu_{o}^{2}} \\
& +4 c_{b}^{2} s_{b}^{2} c_{t}^{2} s_{t}^{2} \log \frac{\tilde{m}_{t_{1}}^{2}+\tilde{m}_{t_{2}}^{2}+\tilde{m}_{b_{1}}^{2}+\tilde{m}_{b_{2}}^{2}}{4 \mu_{o}^{2}}, \\
& g_{9}=\log \frac{3 \tilde{M}_{1}^{+2}+\tilde{M}_{2}^{o 2}}{4 \mu_{o}^{2}}+\frac{1}{4} \log \frac{3 \tilde{M}_{2}^{+2}+\tilde{M}_{3}^{o 2}}{4 \mu_{o}^{2}}+\frac{1}{4} \log \frac{3 \tilde{M}_{2}^{+2}+\tilde{M}_{4}^{o 2}}{4 \mu_{o}^{2}} \\
& g_{10}=c_{W}^{2} \log \frac{3 \tilde{M}_{1}^{+2}+\tilde{M}_{2}^{o 2}}{4 \mu_{o}^{2}}+\frac{1}{4} \log \frac{2 \tilde{M}_{2}^{+2}+\tilde{M}_{3}^{o 2}+\tilde{M}_{4}^{o 2}}{4 \mu_{o}^{2}} \\
& -\frac{1}{8}\left(2 s_{W}^{2}-1\right)\left(\log \frac{3 \tilde{M}_{2}^{+2}+\tilde{M}_{3}^{o 2}}{4 \mu_{o}^{2}}+\log \frac{3 \tilde{M}_{2}^{+2}+\tilde{M}_{4}^{o 2}}{4 \mu_{o}^{2}}\right), \\
& g_{11}=-4 c_{W}^{4} \log \frac{3 \tilde{M}_{1}^{+2}+\tilde{M}_{2}^{o 2}}{4 \mu_{o}^{2}}-\frac{1}{4}\left(2 s_{W}^{2}-1\right)^{2}\left(\log \frac{3 \tilde{M}_{2}^{+2}+\tilde{M}_{3}^{o 2}}{4 \mu_{o}^{2}}+\log \frac{3 \tilde{M}_{2}^{+2}+\tilde{M}_{4}^{o 2}}{4 \mu_{o}^{2}}\right) \\
& +\left(2 s_{W}^{2}-1\right) \log \frac{2 \tilde{M}_{2}^{+2}+\tilde{M}_{3}^{o 2}+\tilde{M}_{4}^{o 2}}{4 \mu_{o}^{2}}-\frac{1}{4} \log \frac{\tilde{M}_{2}^{+2}+2 \tilde{M}_{3}^{o 2}+\tilde{M}_{4}^{o 2}}{4 \mu_{o}^{2}} \\
& -\frac{1}{4} \log \frac{\tilde{M}_{2}^{+2}+\tilde{M}_{3}^{o 2}+2 \tilde{M}_{4}^{o 2}}{4 \mu_{o}^{2}} \\
& g_{12}=-8 \log \frac{\tilde{M}_{1}^{+2}+\tilde{M}_{2}^{o 2}}{2 \mu_{o}^{2}}-\frac{1}{2} \log \frac{\tilde{M}_{2}^{+2}+\tilde{M}_{3}^{o 2}}{2 \mu_{o}^{2}}-\frac{1}{2} \log \frac{\tilde{M}_{2}^{+2}+\tilde{M}_{4}^{o 2}}{2 \mu_{o}^{2}} \\
& -\log \frac{2 \tilde{M}_{2}^{+2}+\tilde{M}_{3}^{o 2}+\tilde{M}_{4}^{o 2}}{4 \mu_{o}^{2}} .
\end{aligned}
$$

\section{Appendix C.}

This appendix is devoted to present the exact results to one loop for the three and four point sfermions contributions as well as the three point inos contributions which are denoted in the text by $\Delta \Gamma_{\mu \nu \sigma}^{A W^{+} W_{\tilde{q}}^{-}}, \Delta \Gamma_{\mu \nu \sigma}^{Z W^{+} W_{\tilde{q}}^{-}}$, and $\Delta \Gamma_{\mu \nu \sigma \lambda}^{A A W^{+} W_{\tilde{q}}^{-}}, \Delta \Gamma_{\mu \nu \sigma \lambda}^{A Z W_{\tilde{q}}^{+} W_{\tilde{q}}^{-}}, \Delta \Gamma_{\mu \nu \sigma \lambda}^{Z Z W_{\tilde{q}}^{+} W_{\tilde{q}}^{-}}, \Delta \Gamma_{\mu \nu \sigma \lambda}^{W^{+} W^{-} W_{\tilde{q}}^{+} W^{-}}$and $\Delta \Gamma_{\mu \nu \sigma}^{A W^{+} W_{\tilde{\chi}}^{-}}, \Delta \Gamma_{\mu \nu \sigma}^{Z W^{+} W_{\tilde{\chi}}^{-}}$respectively. The exact formulae for the four point inos contributions have 
also been computed by us and are available upon request, but they are not shown here due to their extreme length. The momenta assignements for the external gauge bosons are $V_{1}^{\mu}(p) V_{2}^{\nu}(k) V_{3}^{\sigma}(r)$ in the three point functions, $\Delta \Gamma_{\mu \nu \sigma}^{V_{1} V_{2} V_{3}}$, and $V_{1}^{\mu}(p) V_{2}^{\nu}(q) V_{3}^{\sigma}(r) V_{4}^{\lambda}(t)$ in the four point functions, $\Delta \Gamma_{\mu \nu \sigma \lambda}^{V_{1} V_{2} V_{3} V_{4}}$, and the convention is with all the external momenta outgoing. For shortness we omit here the arguments in the Feynman integrals since the notation we have chosen for them is self-explanatory. Thus, for instance,

$$
J_{\mu \nu \sigma \lambda}^{a b c d} \equiv J_{\mu \nu \sigma \lambda}^{a b c d}\left(p, k, r, t, \tilde{m}_{f_{a}}, \tilde{m}_{f_{b}}, \tilde{m}_{f_{c}}\right), J_{\lambda \nu \sigma \mu}^{a b c d} \equiv J_{\lambda \nu \sigma \mu}^{a b c d}\left(t, k, r, p, \tilde{m}_{f_{a}}, \tilde{m}_{f_{b}}, \tilde{m}_{f_{c}}\right),
$$

and so on.

- Three point sfermions contributions.

$$
\begin{aligned}
& \Delta \Gamma_{\mu \nu \sigma}^{A W^{+} W_{\tilde{q}}^{-}}=-e g^{2} N_{c} \frac{\pi^{2}}{2} \sum_{\tilde{f}}\left\{\sum _ { a , b } \left[\left(\widehat{Q}_{f}\right)_{a b}\left(\Sigma_{f}\right)_{b a} T_{\mu}^{a b} g_{\nu \sigma}+\frac{1}{3}\left(\left(\Sigma_{f}^{t b}\right)_{a b}\left(\Sigma_{f}^{b t}\right)_{b a} T_{\sigma}^{a b} g_{\mu \sigma}\right.\right.\right. \\
& \left.\left.+\left(\sum_{f}^{b t}\right)_{a b}\left(\sum_{f}^{t b}\right)_{b a} T_{\nu}^{a b} g_{\mu \nu}\right)\right]-\frac{1}{3} \sum_{a, b, c}\left[\left(\widehat{Q}_{f}\right)_{a b}\left(\sum_{f}^{t b}\right)_{b c}\left(\sum_{f}^{b t}\right)_{c a} T_{\mu \nu \sigma}^{a b c}\right. \\
& +\left(\widehat{Q}_{f}\right)_{a b}\left(\Sigma_{f}^{b t}\right)_{b c}\left(\Sigma_{f}^{t b}\right)_{c a} T_{\mu \sigma \nu}^{a b c}+\left(\Sigma_{f}^{t b}\right)_{a b}\left(\widehat{Q}_{f}\right)_{b c}\left(\Sigma_{f}^{b t}\right)_{c a} T_{\nu \mu \sigma}^{a b c}+\left(\Sigma_{f}^{b t}\right)_{a b}\left(\widehat{Q}_{f}\right)_{b c}\left(\Sigma_{f}^{t b}\right)_{c a} T_{\sigma \mu \nu}^{a b c} \\
& \left.\left.+\left(\sum_{f}^{t b}\right)_{a b}\left(\sum_{f}^{b t}\right)_{b c}\left(\widehat{Q}_{f}\right)_{c a} T_{\nu \sigma \mu}^{a b c}+\left(\sum_{f}^{b t}\right)_{a b}\left(\Sigma_{f}^{t b}\right)_{a b}\left(\widehat{Q}_{f}\right)_{a b} T_{\sigma \nu \mu}^{a b c}\right]\right\} \\
& \Delta \Gamma_{\mu \nu \sigma}^{Z W^{+} W^{-}}=-g^{3} N_{c} \frac{\pi^{2}}{2 c_{W}} \sum_{\tilde{f}}\left\{\sum _ { a , b } \left[\left(\widehat{G}_{f}\right)_{a b}\left(\Sigma_{f}\right)_{a b} T_{\mu}^{a b} g_{\nu \sigma}-\frac{1}{3} s_{W}^{2}\left(\left(\Sigma_{f}^{t b}\right)_{a b}\left(\Sigma_{f}^{b t}\right)_{b a} T_{\sigma}^{a b} g_{\mu \sigma}\right.\right.\right. \\
& \left.\left.+\left(\sum_{f}^{b t}\right)_{a b}\left(\Sigma_{f}^{t b}\right)_{b a} T_{\nu}^{a b} g_{\mu \nu}\right)\right]-\frac{1}{3} \sum_{a, b, c}\left[\left(\widehat{G}_{f}\right)_{a b}\left(\Sigma_{f}^{t b}\right)_{b c}\left(\Sigma_{f}^{b t}\right)_{c a} T_{\mu \nu \sigma}^{a b c}\right. \\
& +\left(\widehat{G}_{f}\right)_{a b}\left(\sum_{f}^{b t}\right)_{b c}\left(\sum_{f}^{t b}\right)_{c a} T_{\sigma \mu \nu}^{a b c}+\left(\sum_{f}^{t b}\right)_{a b}\left(\widehat{G}_{f}\right)_{b c}\left(\sum_{f}^{b t}\right)_{c a} T_{\nu \mu \sigma}^{a b c}+\left(\sum_{f}^{b t}\right)_{a b}\left(\widehat{G}_{f}\right)_{b c}\left(\Sigma_{f}^{t b}\right)_{c a} T_{\sigma \mu \nu}^{a b c} \\
& \left.\left.+\left(\Sigma_{f}^{t b}\right)_{a b}\left(\Sigma_{f}^{b t}\right)_{b c}\left(\widehat{G}_{f}\right)_{c a} T_{\nu \sigma \mu}^{a b c}+\left(\Sigma_{f}^{b t}\right)_{a b}\left(\Sigma_{f}^{t b}\right)_{b c}\left(\widehat{G}_{f}\right)_{c a}\right] T_{\sigma \nu \mu}^{a b c}\right\}
\end{aligned}
$$

- Four point sfermions contributions.

$$
\begin{aligned}
& \Delta \Gamma_{\mu \nu \sigma \lambda}^{A A W_{\tilde{q}}^{+} W^{-}}=e^{2} g^{2} \pi^{2} N_{c} \sum_{\tilde{f}}\left\{\frac { 1 } { 2 } \sum _ { a , b } \left[\left(\left(\widehat{Q}_{f}^{2}\right)_{a b}\left(\Sigma_{f}\right)_{b a}+\left(\Sigma_{f}\right)_{a b}\left(\widehat{Q}_{f}^{2}\right)_{b a}\right) g_{\mu \nu} g_{\sigma \lambda} J_{p+k}^{a b}\right.\right. \\
& \left.+\frac{1}{9}\left(\left(\Sigma_{f}^{t b}\right)_{a b}\left(\sum_{f}^{b t}\right)_{b a} g_{\mu \sigma} g_{\nu \lambda} J_{p+r}^{a b}+\left(\Sigma_{f}^{b t}\right)_{a b}\left(\Sigma_{f}^{t b}\right)_{b a} g_{\mu \lambda} g_{\nu \sigma} J_{p+t}^{a b}\right)\right] \\
& -\sum_{a, b, c}\left[\left(\widehat{Q}_{f}\right)_{a b}\left(\widehat{Q}_{f}\right)_{b c}\left(\Sigma_{f}\right)_{c a} J_{\mu \nu}^{a b c} g_{\sigma \lambda}+\left(\sum_{f}^{t b}\right)_{a b}\left(\sum_{f}^{b t}\right)_{b c}\left(\widehat{Q}_{f}^{2}\right)_{c a} J_{\lambda \sigma}^{a b c} g_{\mu \nu}\right. \\
& +\left(\sum_{f}^{b t}\right)_{a b}\left(\Sigma_{f}^{t b}\right)_{b c}\left(\widehat{Q}_{f}^{2}\right)_{c a} J_{\sigma \lambda}^{a b c} g_{\mu \nu}+\frac{1}{3}\left(\left(\widehat{Q}_{f}\right)_{a b}\left(\Sigma_{f}^{b t}\right)_{b c}\left(\sum_{f}^{t b}\right)_{c a} J_{\mu \lambda}^{a b c} g_{\nu \sigma}\right. \\
& \left.\left.+\left(\sum_{f}^{t b}\right)_{a b}\left(\widehat{Q}_{f}\right)_{b c}\left(\Sigma_{f}^{b t}\right)_{c a} J_{\sigma \nu}^{a b c} g_{\mu \lambda}+\left(\Sigma_{f}^{b t}\right)_{a b}\left(\widehat{Q}_{f}\right)_{b c}\left(\Sigma_{f}^{t b}\right)_{c a} J_{\lambda \nu}^{a b c} g_{\mu \sigma}+\left(\widehat{Q}_{f}\right)_{a b}\left(\Sigma_{f}^{t b}\right)_{b c}\left(\Sigma_{f}^{b t}\right)_{c a} J_{\mu \sigma}^{a b c} g_{\nu \lambda}\right)\right]
\end{aligned}
$$




$$
\begin{aligned}
& +\frac{1}{4} \sum_{a, b, c, d}\left[\left(\widehat{Q}_{f}\right)_{a b}\left(\widehat{Q}_{f}\right)_{b c}\left(\left(\Sigma_{f}^{t b}\right)_{c d}\left(\sum_{f}^{b t}\right)_{d a} J_{\mu \nu \sigma \lambda}^{a b c d}+\left(\sum_{f}^{b t}\right)_{c d}\left(\Sigma_{f}^{t b}\right)_{d a} J_{\mu \nu \lambda \sigma}^{a b c d}\right)\right. \\
& +\left(\widehat{Q}_{f}\right)_{a b}\left(\Sigma_{f}^{t b}\right)_{b c}\left(\widehat{Q}_{f}\right)_{c d}\left(\Sigma_{f}^{b t}\right)_{d a} J_{\mu \sigma \nu \lambda}^{a b c d}+\left(\widehat{Q}_{f}\right)_{a b}\left(\sum_{f}^{b t}\right)_{b c}\left(\widehat{Q}_{f}\right)_{c d}\left(\Sigma_{f}^{t b}\right)_{d a} J_{\mu \lambda \nu \sigma}^{a b c d} \\
& +\left(\widehat{Q}_{f}\right)_{a b}\left(\left(\Sigma_{f}^{t b}\right)_{b c}\left(\sum_{f}^{b t}\right)_{c d} J_{\mu \sigma \lambda \nu}^{a b c d}+\left(\sum_{f}^{b t}\right)_{b c}\left(\Sigma_{f}^{t b}\right)_{c d} J_{\mu \lambda \sigma \nu}^{a b c d}\right)\left(\widehat{Q}_{f}\right)_{d a} \\
& +\left(\sum_{f}^{t b}\right)_{a b}\left(\widehat{Q}_{f}\right)_{b c}\left(\Sigma_{f}^{b t}\right)_{c d}\left(\widehat{Q}_{f}\right)_{d a} J_{\sigma \nu \lambda \mu}^{a b c d}+\left(\Sigma_{f}^{b t}\right)_{a b}\left(\widehat{Q}_{f}\right)_{b c}\left(\Sigma_{f}^{t b}\right)_{c d}\left(\widehat{Q}_{f}\right)_{d a} J_{\lambda \nu \sigma \mu}^{a b c d} \\
& \left(\left(\Sigma_{f}^{t b}\right)_{a b}\left(\sum_{f}^{b t}\right)_{b c} J_{\sigma \lambda \mu \nu}^{a b c d}+\left(\sum_{f}^{b t}\right)_{a b}\left(\Sigma_{f}^{t b}\right)_{b c} J_{\lambda \sigma \mu \nu}^{a b c d}\right)\left(\widehat{Q}_{f}\right)_{c d}\left(\widehat{Q}_{f}\right)_{d a} \\
& \left.\left.+\left(\Sigma_{f}^{t b}\right)_{a b}\left(\widehat{Q}_{f}\right)_{b c}\left(\widehat{Q}_{f}\right)_{c d}\left(\sum_{f}^{b t}\right)_{d a} J_{\sigma \nu \mu \lambda}^{a b c d}+\left(\Sigma_{f}^{b t}\right)_{a b}\left(\widehat{Q}_{f}\right)_{b c}\left(\widehat{Q}_{f}\right)_{c d}\left(\Sigma_{f}^{t b}\right)_{d a} J_{\lambda \nu \mu \sigma}^{a b c d}\right]\right\} \\
& \Delta \Gamma_{\mu \nu \sigma \lambda}^{A Z W_{\tilde{q}}^{+} W^{-}}=\frac{e g^{3}}{c_{W}} \frac{\pi^{2}}{2} N_{c} \sum_{\tilde{f}}\left\{\sum _ { a , b } \left[\left(\left(\widehat{Q}_{f} \widehat{G}_{f}\right)_{a b}\left(\Sigma_{f}\right)_{b a} J_{p+k}^{a b}+\left(\Sigma_{f}\right)_{a b}\left(\widehat{Q}_{f} \widehat{G}_{f}\right)_{b a} J_{r+t}^{a b}\right) g_{\mu \nu} g_{\sigma \lambda}\right.\right. \\
& -\frac{s_{W}^{2}}{18}\left(\left(\left(\Sigma_{f}^{t b}\right)_{a b}\left(\sum_{f}^{b t}\right)_{b a} J_{p+r}^{a b}+\left(\Sigma_{f}^{b t}\right)_{a b}\left(\Sigma_{f}^{t b}\right)_{b a} J_{k+r}^{a b}\right) g_{\mu \sigma} g_{\nu \lambda}\right. \\
& \left.\left.+\left(\left(\sum_{f}^{t b}\right)_{a b}\left(\sum_{f}^{b t}\right)_{b a} J_{k+r}^{a b}+\left(\sum_{f}^{b t}\right)_{a b}\left(\Sigma_{f}^{t b}\right)_{b a} J_{p+t}^{a b}\right) g_{\mu \lambda} g_{\nu \sigma}\right)\right] \\
& -\sum_{a, b, c}\left[\left(\left(\widehat{Q}_{f}\right)_{a b}\left(\widehat{G}_{f}\right)_{b c} J_{\mu \nu}^{a b c} g_{\sigma \lambda}+\left(\widehat{G}_{f}\right)_{a b}\left(\widehat{Q}_{f}\right)_{b c} J_{\nu \mu}^{a b c} g_{\sigma \lambda}\right)\left(\Sigma_{f}\right)_{c a}\right. \\
& \left.+2\left(\left(\sum_{f}^{t b}\right)_{a b}\left(\Sigma_{f}^{b t}\right)_{b c} J_{\sigma \lambda}^{a b c} g_{\mu \nu}+\left(\sum_{f}^{b t}\right)_{a b} J_{\lambda \sigma}^{a b c} g_{\nu \mu}\right)\left(\Sigma_{f}^{t b}\right)_{b c}\right)\left(\widehat{Q}_{f} \widehat{G}_{f}\right)_{b a} \\
& -\frac{s_{W}^{2}}{3}\left(\left(\widehat{Q}_{f}\right)_{a b}\left(\Sigma_{f}^{b t}\right)_{b c}\left(\Sigma_{f}^{t b}\right)_{c a} J_{\mu \lambda}^{a b c} g_{\nu \sigma}+\left(\Sigma_{f}^{b t}\right)_{a b}\left(\widehat{Q}_{f}\right)_{b c}\left(\Sigma_{f}^{t b}\right)_{c a} J_{\lambda \mu}^{a b c} g_{\nu \sigma}\right. \\
& \left.+\left(\widehat{Q}_{f}\right)_{a b}\left(\Sigma_{f}^{t b}\right)_{b c}\left(\Sigma_{f}^{b t}\right)_{c a} J_{\mu \sigma}^{a b c} g_{\nu \lambda}+\left(\Sigma_{f}^{t b}\right)_{a b}\left(\widehat{Q}_{f}\right)_{b c}\left(\sum_{f}^{b t}\right)_{c a} J_{\sigma \mu}^{a b c} g_{\nu \lambda}\right) \\
& +\frac{1}{3}\left(\left(\widehat{G}_{f}\right)_{a b}\left(\Sigma_{f}^{t b}\right)_{b c}\left(\Sigma_{f}^{b t}\right)_{c a} J_{\nu \sigma}^{a b c} g_{\mu \lambda}+\left(\Sigma_{f}^{t b}\right)_{a b}\left(\widehat{G}_{f}\right)_{b c}\left(\sum_{f}^{b t}\right)_{c a} J_{\sigma \nu}^{a b c} g_{\mu \lambda}\right. \\
& \left.\left.+\left(\widehat{G}_{f}\right)_{a b}\left(\sum_{f}^{b t}\right)_{b c}\left(\sum_{f}^{t b}\right)_{c a} J_{\nu \lambda}^{a b c} g_{\mu \sigma}+\left(\Sigma_{f}^{b t}\right)_{a b}\left(\widehat{G}_{f}\right)_{b c}\left(\Sigma_{f}^{t b}\right)_{c a} J_{\lambda \nu}^{a b c} g_{\mu \sigma}\right)\right] \\
& +\frac{1}{4} \sum_{a, b, c, d}\left[\left(\left(\widehat{Q}_{f}\right)_{a b}\left(\widehat{G}_{f}\right)_{b c} J_{\mu \nu \sigma \lambda}^{a b c d}+\left(\widehat{G}_{f}\right)_{a b}\left(\widehat{Q}_{f}\right)_{b c} J_{\nu \mu \sigma \lambda}^{a b c d}\right)\left(\Sigma_{f}^{t b}\right)_{c d}\left(\sum_{f}^{b t}\right)_{d a}\right. \\
& +\left(\left(\widehat{Q}_{f}\right)_{a b}\left(\widehat{G}_{f}\right)_{b c} J_{\mu \nu \lambda \sigma}^{a b c d}+\left(\widehat{G}_{f}\right)_{a b}\left(\widehat{Q}_{f}\right)_{b c} J_{\nu \mu \lambda \sigma}^{a b c d}\right)\left(\Sigma_{f}^{b t}\right)_{c d}\left(\Sigma_{f}^{t b}\right)_{d a} \\
& +\left(\widehat{Q}_{f}\right)_{a b}\left(\Sigma_{f}^{t b}\right)_{b c}\left(\widehat{G}_{f}\right)_{c d}\left(\Sigma_{f}^{b t}\right)_{d a} J_{\mu \sigma \nu \lambda}^{a b c d}+\left(\widehat{G}_{f}\right)_{a b}\left(\Sigma_{f}^{t b}\right)_{b c}\left(\widehat{Q}_{f}\right)_{c d}\left(\Sigma_{f}^{b t}\right)_{d a} J_{\nu \sigma \mu \lambda}^{a b c d} \\
& +\left(\widehat{Q}_{f}\right)_{a b}\left(\sum_{f}^{b t}\right)_{b c}\left(\widehat{G}_{f}\right)_{c d}\left(\Sigma_{f}^{t b}\right)_{d a} J_{\mu \lambda \nu \sigma}^{a b c d}+\left(\widehat{G}_{f}\right)_{a b}\left(\Sigma_{f}^{b t}\right)_{b c}\left(\widehat{Q}_{f}\right)_{c d}\left(\Sigma_{f}^{t b}\right)_{d a} J_{\nu \lambda \mu \sigma}^{a b c d} \\
& +\left(\widehat{Q}_{f}\right)_{a b}\left(\left(\Sigma_{f}^{t b}\right)_{b c}\left(\sum_{f}^{b t}\right)_{c d} J_{\mu \sigma \lambda \nu}^{a b c d}+\left(\Sigma_{f}^{b t}\right)_{b c}\left(\Sigma_{f}^{t b}\right)_{c d} J_{\mu \lambda \sigma \nu}^{a b c d}\right)\left(\widehat{G}_{f}\right)_{d a} \\
& +\left(\widehat{G}_{f}\right)_{a b}\left(\left(\Sigma_{f}^{t b}\right)_{b c}\left(\Sigma_{f}^{b t}\right)_{c d} J_{\nu \sigma \lambda \mu}^{a b c d}+\left(\Sigma_{f}^{b t}\right)_{b c}\left(\Sigma_{f}^{t b}\right)_{c d} J_{\nu \lambda \sigma \mu}^{a b c d}\right)\left(\widehat{Q}_{f}\right)_{d a} \\
& +\left(\Sigma_{f}^{t b}\right)_{a b}\left(\widehat{Q}_{f}\right)_{b c}\left(\Sigma_{f}^{b t}\right)_{c d}\left(\widehat{G}_{f}\right)_{d a} J_{\sigma \mu \lambda \nu}^{a b c d}+\left(\Sigma_{f}^{t b}\right)_{a b}\left(\widehat{G}_{f}\right)_{b c}\left(\Sigma_{f}^{b t}\right)_{c d}\left(\widehat{Q}_{f}\right)_{d a} J_{\sigma \nu \lambda \mu}^{a b c d} \\
& +\left(\Sigma_{f}^{b t}\right)_{a b}\left(\widehat{Q}_{f}\right)_{b c}\left(\Sigma_{f}^{t b}\right)_{c d}\left(\widehat{G}_{f}\right)_{d a} J_{\lambda \mu \sigma \nu}^{a b c d}+\left(\Sigma_{f}^{b t}\right)_{a b}\left(\widehat{G}_{f}\right)_{b c}\left(\Sigma_{f}^{t b}\right)_{c d}\left(\widehat{Q}_{f}\right)_{d a} J_{\lambda \nu \sigma \mu}^{a b c d} \\
& +\left(\left(\sum_{f}^{t b}\right)_{a b}\left(\sum_{f}^{b t}\right)_{b c} J_{\sigma \lambda \nu \mu}^{a b c d}+\left(\sum_{f}^{b t}\right)_{a b}\left(\Sigma_{f}^{t b}\right)_{b c} J_{\lambda \sigma \nu \mu}^{a b c d}\right)\left(\widehat{Q}_{f}\right)_{c d}\left(\widehat{G}_{f}\right)_{d a} \\
& +\left(\left(\sum_{f}^{t b}\right)_{a b}\left(\sum_{f}^{b t}\right)_{b c} J_{\sigma \lambda \mu \nu}^{a b c d}+\left(\sum_{f}^{b t}\right)_{a b}\left(\Sigma_{f}^{t b}\right)_{b c} J_{\lambda \sigma \mu \nu}^{a b c d}\right)\left(\widehat{G}_{f}\right)_{c d}\left(\widehat{Q}_{f}\right)_{d a}
\end{aligned}
$$




$$
\begin{aligned}
& +\left(\Sigma_{f}^{t b}\right)_{a b}\left(\left(\widehat{Q}_{f}\right)_{c d}\left(\widehat{G}_{f}\right)_{d a} J_{\sigma \mu \nu \lambda}^{a b c d}+\left(\widehat{G}_{f}\right)_{c d}\left(\widehat{Q}_{f}\right)_{d a} J_{\sigma \nu \mu \lambda}^{a b c d}\right)\left(\Sigma_{f}^{b t}\right)_{d a} \\
& \left.\left.+\left(\sum_{f}^{b t}\right)_{a b}\left(\left(\widehat{Q}_{f}\right)_{c d}\left(\widehat{G}_{f}\right)_{d a} J_{\lambda \mu \nu \sigma}^{a b c d}+\left(\widehat{G}_{f}\right)_{c d}\left(\widehat{Q}_{f}\right)_{d a} J_{\lambda \nu \mu \sigma}^{a b c d}\right)\left(\Sigma_{f}^{t b}\right)_{d a}\right]\right\} \\
& \Delta \Gamma_{\mu \nu \sigma \lambda}^{Z Z W_{\tilde{q}}^{+} W^{-}}=\frac{g^{4}}{c_{W}^{2}} \pi^{2} N_{c} \sum_{\tilde{f}}\left\{\frac { 1 } { 2 } \sum _ { a , b } \left[\left(\left(\widehat{G}_{f}^{2}\right)_{a b}\left(\Sigma_{f}\right)_{b a}+\left(\Sigma_{f}\right)_{a b}\left(\widehat{G}_{f}^{2}\right)_{b a}\right) g_{\mu \nu} g_{\sigma \lambda} J_{p+k}^{a b}\right.\right. \\
& \left.+\frac{s_{W}^{4}}{9}\left(\left(\Sigma_{f}^{t b}\right)_{a b}\left(\sum_{f}^{b t}\right)_{b a} g_{\mu \sigma} g_{\nu \lambda} J_{p+r}^{a b}+\left(\Sigma_{f}^{b t}\right)_{a b}\left(\Sigma_{f}^{t b}\right)_{b a} g_{\mu \lambda} g_{\nu \sigma} J_{p+t}^{a b}\right)\right] \\
& -\sum_{a, b, c}\left[\left(\widehat{G}_{f}\right)_{a b}\left(\widehat{G}_{f}\right)_{b c}\left(\Sigma_{f}\right)_{c a} J_{\mu \nu}^{a b c} g_{\sigma \lambda}+\left(\Sigma_{f}^{t b}\right)_{a b}\left(\Sigma_{f}^{b t}\right)_{b c}\left(\widehat{G}_{f}^{2}\right)_{c a} J_{\lambda \sigma}^{a b c} g_{\mu \nu}\right. \\
& +\left(\sum_{f}^{b t}\right)_{a b}\left(\sum_{f}^{t b}\right)_{b c}\left(\widehat{G}_{f}^{2}\right)_{c a} J_{\sigma \lambda}^{a b c} g_{\mu \nu}-\frac{s_{W}^{2}}{3}\left(\left(\widehat{G}_{f}\right)_{a b}\left(\Sigma_{f}^{b t}\right)_{b c}\left(\sum_{f}^{t b}\right)_{c a} J_{\mu \lambda}^{a b c} g_{\nu \sigma}\right. \\
& \left.\left.+\left(\Sigma_{f}^{t b}\right)_{a b}\left(\widehat{G}_{f}\right)_{b c}\left(\Sigma_{f}^{b t}\right)_{c a} J_{\sigma \nu}^{a b c} g_{\mu \lambda}+\left(\sum_{f}^{b t}\right)_{a b}\left(\widehat{G}_{f}\right)_{b c}\left(\sum_{f}^{t b}\right)_{c a} J_{\lambda \nu}^{a b c} g_{\mu \sigma}+\left(\widehat{G}_{f}\right)_{a b}\left(\Sigma_{f}^{t b}\right)_{b c}\left(\Sigma_{f}^{b t}\right)_{c a} J_{\mu \sigma}^{a b c} g_{\nu \lambda}\right)\right] \\
& +\frac{1}{4} \sum_{a, b, c, d}\left[\left(\widehat{G}_{f}\right)_{a b}\left(\widehat{G}_{f}\right)_{b c}\left(\left(\Sigma_{f}^{t b}\right)_{c d}\left(\Sigma_{f}^{b t}\right)_{d a} J_{\mu \nu \sigma \lambda}^{a b c d}+\left(\sum_{f}^{b t}\right)_{c d}\left(\Sigma_{f}^{t b}\right)_{d a} J_{\mu \nu \lambda \sigma}^{a b c d}\right)\right. \\
& +\left(\widehat{G}_{f}\right)_{a b}\left(\Sigma_{f}^{t b}\right)_{b c}\left(\widehat{G}_{f}\right)_{c d}\left(\Sigma_{f}^{b t}\right)_{d a} J_{\mu \sigma \nu \lambda}^{a b c d}+\left(\widehat{G}_{f}\right)_{a b}\left(\sum_{f}^{b t}\right)_{b c}\left(\widehat{G}_{f}\right)_{c d}\left(\Sigma_{f}^{t b}\right)_{d a} J_{\mu \lambda \nu \sigma}^{a b c d} \\
& +\left(\widehat{G}_{f}\right)_{a b}\left(\left(\Sigma_{f}^{t b}\right)_{b c}\left(\sum_{f}^{b t}\right)_{c d} J_{\mu \sigma \lambda \nu}^{a b c d}+\left(\sum_{f}^{b t}\right)_{b c}\left(\Sigma_{f}^{t b}\right)_{c d} J_{\mu \lambda \sigma \nu}^{a b c d}\right)\left(\widehat{G}_{f}\right)_{d a} \\
& +\left(\Sigma_{f}^{t b}\right)_{a b}\left(\widehat{G}_{f}\right)_{b c}\left(\Sigma_{f}^{b t}\right)_{c d}\left(\widehat{G}_{f}\right)_{d a} J_{\sigma \nu \lambda \mu}^{a b c d}+\left(\Sigma_{f}^{b t}\right)_{a b}\left(\widehat{G}_{f}\right)_{b c}\left(\Sigma_{f}^{t b}\right)_{c d}\left(\widehat{G}_{f}\right)_{d a} J_{\lambda \nu \sigma \mu}^{a b c d} \\
& \left(\left(\Sigma_{f}^{t b}\right)_{a b}\left(\sum_{f}^{b t}\right)_{b c} J_{\sigma \lambda \mu \nu}^{a b c d}+\left(\sum_{f}^{b t}\right)_{a b}\left(\Sigma_{f}^{t b}\right)_{b c} J_{\lambda \sigma \mu \nu}^{a b c d}\right)\left(\widehat{G}_{f}\right)_{c d}\left(\widehat{G}_{f}\right)_{d a} \\
& \left.\left.+\left(\Sigma_{f}^{t b}\right)_{a b}\left(\widehat{G}_{f}\right)_{b c}\left(\widehat{G}_{f}\right)_{c d}\left(\sum_{f}^{b t}\right)_{d a} J_{\sigma \nu \mu \lambda}^{a b c d}+\left(\Sigma_{f}^{b t}\right)_{a b}\left(\widehat{G}_{f}\right)_{b c}\left(\widehat{G}_{f}\right)_{c d}\left(\Sigma_{f}^{t b}\right)_{d a} J_{\lambda \nu \mu \sigma}^{a b c d}\right]\right\} \\
& \Delta \Gamma_{\mu \nu \sigma \lambda}^{W_{\tilde{q}}^{+} W^{-}} W_{\tilde{q}}^{+} W^{-}=g^{4} \pi^{2} N_{c} \sum_{\tilde{f}}\left\{\frac{1}{2} \sum_{a, b}\left[\left(\Sigma_{f}\right)_{a b}\left(\Sigma_{f}\right)_{b a} g_{\mu \lambda} g_{\nu \sigma}\right] J_{p+k}^{a b}\right. \\
& -\sum_{a, b, c}\left[\left(\left(\Sigma_{f}^{t b}\right)_{a b}\left(\sum_{f}^{b t}\right)_{b c} J_{\mu \nu}^{a b c} g_{\sigma \lambda}++\left(\Sigma_{f}^{b t}\right)_{a b}\left(\Sigma_{f}^{t b}\right)_{b c} J_{\nu \mu}^{a b c} g_{\sigma \lambda}\right)\left(\Sigma_{f}\right)_{c a}\right] \\
& +\frac{1}{4} \sum_{a, b, c, d}\left[\left(\Sigma_{f}^{t b}\right)_{a b}\left(\Sigma_{f}^{t b}\right)_{b c}\left(\Sigma_{f}^{b t}\right)_{c d}\left(\Sigma_{f}^{b t}\right)_{d a} J_{\mu \sigma \nu \lambda}^{a b c d}+\left(\sum_{f}^{b t}\right)_{a b}\left(\Sigma_{f}^{b t}\right)_{b c}\left(\Sigma_{f}^{t b}\right)_{c d}\left(\Sigma_{f}^{t b}\right)_{d a} J_{\lambda \nu \sigma \mu}^{a b c d}\right. \\
& +\left(\Sigma_{f}^{t b}\right)_{a b}\left(\sum_{f}^{b t}\right)_{b c}\left(\left(\Sigma_{f}^{t b}\right)_{c d}\left(\Sigma_{f}^{b t}\right)_{d a} J_{\mu \nu \sigma \lambda}^{a b c d}+\left(\Sigma_{f}^{b t}\right)_{c d}\left(\Sigma_{f}^{t b}\right)_{d a} J_{\mu \nu \lambda \sigma}^{a b c d}\right) \\
& \left.\left.+\left(\sum_{f}^{b t}\right)_{a b}\left(\Sigma_{f}^{t b}\right)_{b c}\left(\left(\Sigma_{f}^{t b}\right)_{c d}\left(\Sigma_{f}^{b t}\right)_{d a} J_{\nu \mu \sigma \lambda}^{a b c d}+\left(\sum_{f}^{b t}\right)_{c d}\left(\Sigma_{f}^{t b}\right)_{d a} J_{\nu \mu \lambda \sigma}^{a b c d}\right)\right]\right\}
\end{aligned}
$$

- Three point inos contributions.

$$
\begin{aligned}
\Delta \Gamma_{\mu \nu \sigma}^{A W^{+} W^{-}} & =-\frac{e g^{2}}{8 \pi^{2}} \sum_{i=1}^{4} \sum_{j, k=1}^{2} \delta_{j k}\left\{\left(O_{L_{i j}} O_{L_{k i}}^{+}+O_{R_{i j}} O_{R_{k i}}^{+}\right)\left[\mathcal{T}_{\sigma \mu \nu}^{i j k}+\tilde{M}_{j}^{+} \tilde{M}_{k}^{+} \mathcal{I}_{\sigma \mu \nu}^{i j k}\right]\right. \\
& \left.+\left(O_{L_{i j}} O_{R_{k i}}^{+}+O_{R_{i j}} O_{L_{k i}}^{+}\right)\left[\tilde{M}_{i}^{o} \tilde{M}_{k}^{+} \mathcal{P}_{\sigma \mu \nu}^{i j k}+\tilde{M}_{i}^{o} \tilde{M}_{j}^{+} \mathcal{J}_{\sigma \mu \nu}^{i j k}\right]\right\}
\end{aligned}
$$




$$
\begin{aligned}
\Delta \Gamma_{\mu \nu \sigma}^{Z W^{+} W^{-}} & =\frac{g^{3}}{8 \pi^{2}} \frac{1}{c_{W}}\left\{\sum _ { i = 1 } ^ { 4 } \sum _ { j , k = 1 } ^ { 2 } \left[\left(O_{L_{i j}} O_{L_{j k}}^{\prime} O_{L_{k i}}^{+}+O_{R_{i j}} O_{R_{j k}}^{\prime} O_{R_{k i}}^{+}\right) \mathcal{T}_{\sigma \mu \nu}^{i j k}\right.\right. \\
& +\left(O_{L_{i j}} O_{R_{j k}}^{\prime} O_{R_{k i}}^{+}+O_{R_{i j}} O_{L_{j k}}^{\prime} O_{L_{k i}}^{+}\right) \tilde{M}_{i}^{o} \tilde{M}_{j}^{+} \mathcal{J}_{\sigma \mu \nu}^{i j k} \\
& +\left(O_{L_{i j}} O_{R_{j k}}^{\prime} O_{L_{k i}}^{+}+O_{R_{i j}} O_{L_{j k}}^{\prime} O_{R_{k i}}^{+}\right) \tilde{M}_{j}^{+} \tilde{M}_{k}^{+} \mathcal{I}_{\sigma \mu \nu}^{i j k} \\
& \left.+\left(O_{L_{i j}} O_{L_{j k}}^{\prime} O_{R_{k i}}^{+}+O_{R_{i j}} O_{R_{j k}}^{\prime} O_{L_{k i}}^{+}\right) \tilde{M}_{i}^{o} \tilde{M}_{k}^{+} \mathcal{P}_{\sigma \mu \nu}^{i j k}\right] \\
& \quad+\sum_{i, j=1}^{4} \sum_{k=1}^{2}\left[\left(O_{L_{i j}}^{\prime \prime} O_{L_{j k}} O_{L_{k i}}^{+}+O_{R_{i j}}^{\prime \prime} O_{R_{j k}} O_{R_{k i}}^{+}\right) \mathcal{T}_{\mu \sigma \nu}^{i j k}\right. \\
& +\left(O_{L_{i j}^{\prime \prime}}^{\prime \prime} O_{R_{j k}} O_{R_{k i}}^{+}+O_{R_{i j}}^{\prime \prime} O_{L_{j k}} O_{R_{k i}}^{+}\right) \tilde{M}_{i}^{o} \tilde{M}_{j}^{o} \mathcal{J}_{\mu \sigma \nu}^{i j k} \\
+ & \left(O_{L_{i j}}^{\prime \prime} O_{R_{j k}} O_{L_{k i}}^{+}+O_{R_{i j}}^{\prime \prime} O_{L_{j k}} O_{R_{k i}}^{+}\right) \tilde{M}_{j}^{o} \tilde{M}_{k}^{+} \mathcal{I}_{\mu \sigma \nu}^{i j k} \\
+ & \left.\left.\left(O_{L_{i j}}^{\prime \prime} O_{L_{j k}} O_{R_{k i}}^{+}+O_{R_{i j}}^{\prime \prime} O_{R_{j k}} O_{L_{k i}}^{+}\right) \tilde{M}_{i}^{o} \tilde{M}_{k}^{+} \mathcal{P}_{\mu \sigma \nu}^{i j k}\right]\right\}
\end{aligned}
$$

The integrals appearing in the above formula are given in terms of the standard one-loop integrals in appendix A and we refer once more to [16] in order to find the explicit expressions of the coupling matrices.

\section{References}

[1] For an introduction to supersymmetry see, e.g

J.Wess and J.Bagger, Supersymmetry and Supergravity, Princenton University Press, (1983),

N.P.Nilles, Phys. Rep. 110 (1984), 1,

D.Bailin and A.Love,Supersymmetric Gauge Field Theory and String Theory, Institute of Physics Publishing, (1996).

[2] For a pedagogical introduction to the MSSM see,

H.Haber, Introductory low-energy supersymmetry. Published in Boulder TASI-92:589-688, Univ. of Colorado, SCIPP-92/93, hep-ph/9306207.

H.Haber and G.L.Kane, Phys. Rep. 117 (1985), 75.

J.F.Gunion and H.Haber, Nucl. Phys. B272 (1986), 1, Nucl. Phys. B278 (1986), 449.

[3] L.Girardello and M.T.Grisaru, Nucl. Phys. B194 (1982), 65.

[4] P.H.Chankowski et al, Nucl. Phys. B417 (1994), 101.

[5] H.Haber, When are radiative corrections important in the Minimal Supersymmetric Model. (UC, Santa Cruz). SCIPP-93-06, Mar 1993. 52pp. Proceedings of INFN Eloisatron Project: 23rd Workshop: The Decay Properties of SUSY Particles, Erice, Italy, 28 Sep - 4 Oct 1992. In *Erice 1992, 23rd Eloisatron workshop, Properties of SUSY particles*321-372, hepph/9305248. 
[6] D.García and J.Solà, Mod. Phys. Lett. A9 (1994), 211, D.García, R.A.Jiménez,J.Solà and W.Hollik, Nucl. Phys. B427 (1994),53, J.A.Coarasa et al., Eur.Phys.J. C2 (1998), 373,

A.Djouadi et al., Eur.Phys.J. C1 (1998), 149.

[7] T.Inami, C.S.Lim and A.Yamada, Mod. Phys. Lett. A7 (1992), 2789.

[8] H.Haber, Higgs bosons in the Minimal Supersymmetric Model: The influence of radiative corrections. (UC, Santa Cruz). SCIPP-92/31, Published in Perspectives on Higgs Physics, World Scientific Publ., (1992) pp.79-125.

[9] M.Carena, M.Quirós and C.E.M.Wagner, Nucl.Phys. B461 (1996) 407.

[10] T.Appelquist and J.Carazzone, Phys. Rev. D11 (1975), 2856.

[11] For an introduction to the subject of integration of heavy fields and computation of effective actions see, for instance,

A.Dobado et al., Effective Lagrangians for the Standard Model, Springer-Verlag, (1997).

[12] M.Veltman, Act.Phys.Pol. B8 (1977), 475, Nucl. Phys. B123 (1977) 89.

D.R.T.Jones and M.Veltman, Nucl. Phys. B19 (1981) 146.

M.Chanowitz, M.Furman and I.Hinchliffe, Phys. Lett. 78B (1978) 285,Nucl. Phys. B153 (1979) 402.

[13] G.Lin, H.Steger and Y.Yao Phys.Rev. D49 (1994) 2414,

F.Feruglio, L.Maiani and A.Masiero, Nucl. Phys. B387 (1992), 523.

[14] T.Appelquist and C.Bernard, Phys. Rev. D22 (1980) 200,

A.C.Longhitano, Nucl. Phys. B188 (1981), 118; Phys. Rev. D22 (1980), 1166,

M.J.Herrero and E.R.Morales Nucl. Phys. B418 (1994), 431, Nucl. Phys. B437 (1995), 319,

D. Espriu and J.Matias, Phys. Lett. 341B (1995), 332;

S.Dittmaier and C.Grosse-Knetter, Phys. Rev. D52 (1995), 7276, Nucl. Phys. B459 (1996), 497.

[15] G.Giavarini, C.P. Martin and F.Ruiz Ruiz, Nucl. Phys. B381 (1992), 222.

[16] A. Dobado, M.J. Herrero and S.Peñaranda, hep-ph/9710313, (Eur.Phys.J.C (1998) to appear), URL: http://dx.doi.org/10.1007/s100529800993.

[17] G.Passarino and M.Veltman,Nucl. Phys. B160 (1979), 151. 

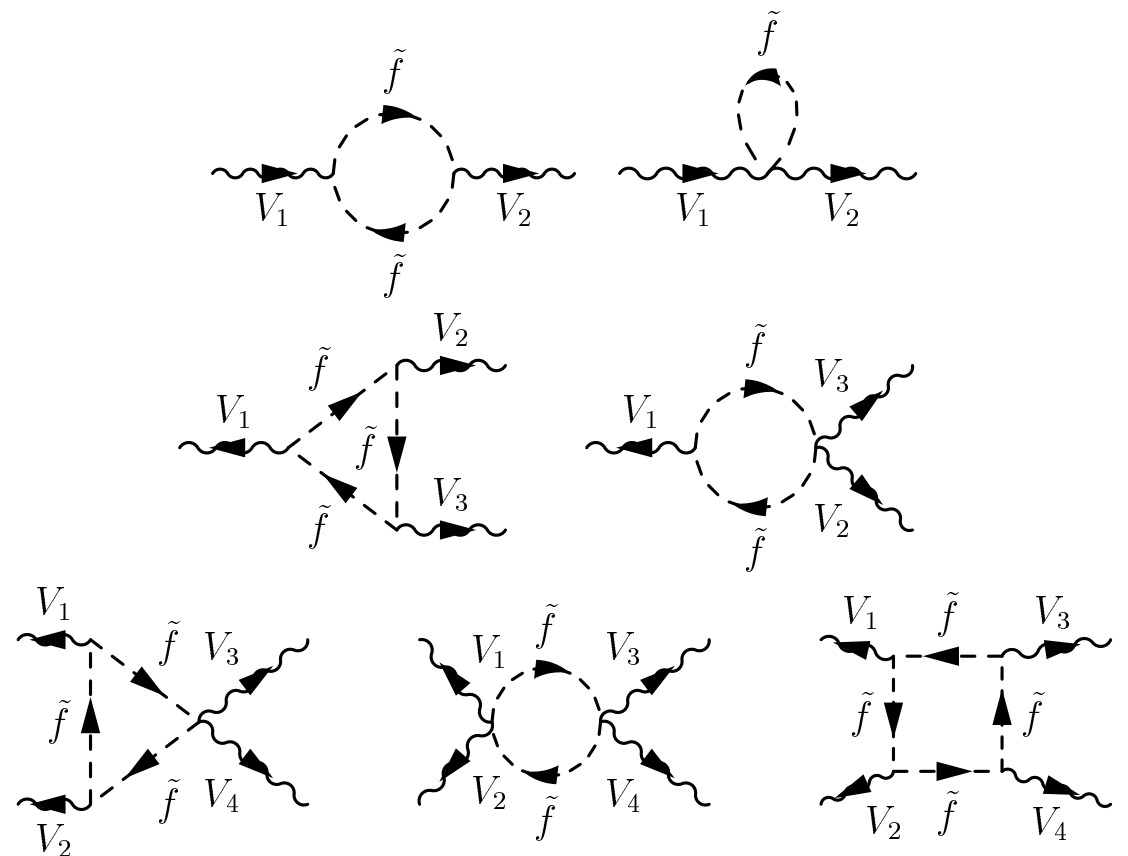

(a)
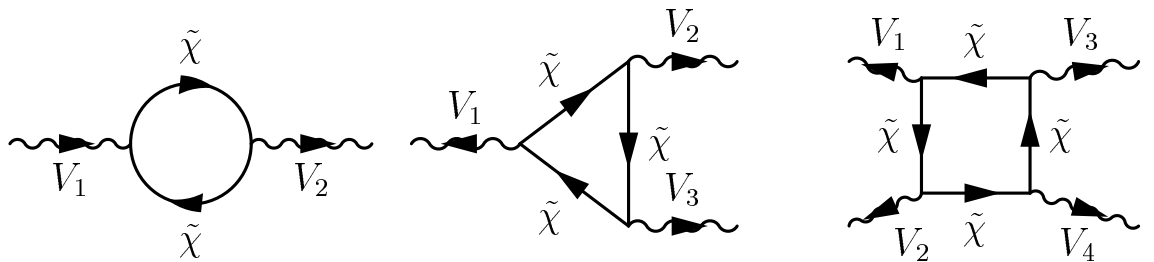

(b)

Fig.1 Generic Feynman diagrams corresponding to one-loop contributions to the two, three and four-point functions.

(a) With sfermions in the loops.

(b) With charginos and neutralinos in the loops. 